Supporting Information for:

\title{
Diastereoselective synthesis of pyrrolidines using a nitrone/cyclopropane cycloaddition: Synthesis of the tetracyclic core of nakadomarin $\mathrm{A}$.
}

lan S. Young, Justin L. Williams and Michael A. Kerr*

Organic Letters 2004 


\section{Experimental}

\section{General}

Melting points are uncorrected. Infrared spectra were obtained as thin films on $\mathrm{NaCl}$ plates. NMR experiments were performed at 400 or $600 \mathrm{MHz}$ spectra were recorded in $\mathrm{CDCl}_{3}$ (referenced to $7.26 \mathrm{ppm}$ for ${ }^{1} \mathrm{H}$ and 77.0 for ${ }^{13} \mathrm{C}$ ). Coupling constants $(\mathrm{J})$ are in $\mathrm{Hz}$. The multiplicities of the signals are described using the following abbreviations: $\mathrm{s}=$ singlet, $\mathrm{d}=$ doublet, $\mathrm{t}=$ triplet, $\mathrm{q}=$ quartet, $\mathrm{m}=$ multiplet, $\mathrm{br}=$ broad. High resolution mass spectra (HRMS) were obtained at $70 \mathrm{eV}$.

Toluene, THF, ether, DMF, methylene chloride were dried and deoxygenated by passing the nitrogen purged solvents through activated alumina columns. All other reagents and solvents were used as purchased from Aldrich, Strem, Caledon or VWR. Reactions progress was followed by thin layer chromatography (TLC) (EM Science, silica gel $60 \mathrm{~F}_{254}$ ) and the developed plates stained using acidic anisaldehyde, phosphomolybidic acid or basic potassium permanganate. Flash chromatography was performed using silica gel purchased from Silicycle Chemical Division Inc. (230-400 mesh)

\section{Pyrrolidine Methodology Section of Experimental}

\section{General Procedure for the Reaction of Nitrones with Cyclopropanes (Step A)}

$\mathrm{Yb}(\mathrm{OTf})_{3} \cdot \mathrm{xH}_{2} \mathrm{O}(5 \mathrm{~mol} \%)$ was added to a solution of a cyclopropane diester (1eqv) and a nitrone (1.2 eqv) in methylene chloride. The reaction was monitored by TLC and following complete consumption of the cyclopropane diester, the reaction mixture was then pre-absorbed onto silica gel. The pure product was obtained after flash column chromatography on silica gel (elution EtOAc in hexanes). Samples could be recrystallized from $\mathrm{CH}_{2} \mathrm{Cl}_{2} /$ hexanes if desired.

Please note that many of the tetrahydro-1,2-oxazines derived from a cycloaddition between a nitrone and cyclopropane have already been characterized in previous papers. Please refer to Young, I.S.; and Kerr, M.A.; Angew. Chem. Int., 2003, 42, 3023-3026, and Young, I.S.; and Kerr, M.A.; Org. Lett., 2004, 6, 139-141 for additional experimental for these compounds.

\section{General Procedure for Reduction of Tetrahydro-1,2-oxazine diesters to diols (Step B)}

Lithium aluminum hydride (2-2.5 eqv of a $1 \mathrm{M}$ solution in ether) was added to a solution of tetrahydro-1,2-oxazine diester (1eqv) in THF at $0^{\circ} \mathrm{C}$ under an argon atmosphere. The reaction was allowed to warm to room temperature where it was stirred for 16hrs. (Complete consumption of starting material could be visualized by TLC after $4 \mathrm{hrs}$ but isolation at this time would often result in substantial mono reduced product.) After $16 \mathrm{hrs}$ the reaction was quenched with water and diluted with $5 \% \mathrm{HCl}$. The reaction was then extracted with 3 times with ethyl acetate. The combined organic extracts were dried over $\mathrm{MgSO}_{4}$, and the solvent removed under reduced pressure. The crude reaction mixture was purified by flash chromatography (elution EtOAc in hexanes). 
General Procedure for Protection of the Diol as the Acetonide (Step C)

A catalytic amount $(20 \mathrm{~mol} \%)$ of p-toluenesulfonic acid mono hydrate $\left(\mathrm{TsOH} \cdot \mathrm{H}_{2} \mathrm{O}\right)$ was added to a solution of the tetrahydro-1,2-oxazine diol (1 eqv) and 2-methoxypropene (3 eqv) in N,Ndimethyl formamide (DMF). The solution was stirred at $\mathrm{rt}$ for $3 \mathrm{hrs}$. The solution was then diluted with water and extracted with 3 times with ether. The combined organic extracts were dried over $\mathrm{MgSO}_{4}$ and the solvent removed under reduced pressure. Further purification was not required.

\section{General Procedure for Reductive cleavage of the N-O Bond (Step D)}

$0.1 \mathrm{M} \mathrm{SmI}_{2}$ (3eqv) was generated in situ by refluxing $\mathrm{Sm}^{\circ}$ (3.85 eqv) and $\mathrm{I}_{2}$ (3.5 eqv) in THF under an argon atmosphere for approximately $45 \mathrm{~min}$ (until solution was entirely blue). The acetonide protected tetrahydro 1,2-oxazine(1eqv) was dissolved in a minimal amount of THF and added to the refluxing solution via a syringe. The reaction was monitored by TLC with typical reaction times ranging from 16-22 hrs. The reaction mixture was diluted with saturated sodium bicarbonate and extracted with ethyl acetate. N-phenyl or N-tol substituted 1,4 amino alcohols were purified by flash chromatography whereas the more polar $\mathrm{N}$-methyl substituted 1,4 amino alcohols were not purified, and used directly in Step $\boldsymbol{E}$.

\section{General Procedure for Pyrrolidine Formation (Step E)}

THF was added dropwise to a mixture of $\mathrm{NaH}$ (sodium hydride) (1.5eqv) and 1,4 amino alcohol (1eqv). The solution was stirred at room temperature for 30 minutes before methanesulfonyl chloride $(\mathrm{MsCl})$ (1.3eqv) was added. The crude mixture was then pre-absorbed onto silica gel and purified by flash chromatography (elution EtOAc in hexanes). 


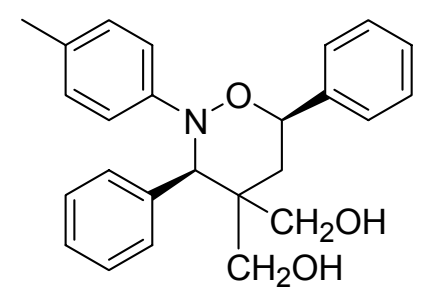

\section{Compound 7ab}

Compound 7ab was prepared from compound 7aa utilizing the general procedure for Step B. Amounts of reagents included $2.0 \mathrm{~g}(4.49 \mathrm{mmol})$ of $7 \mathbf{a a}$ and $11.3 \mathrm{~mL}(11.3 \mathrm{mmol})$ lithium aluminum hydride solution in $40 \mathrm{~mL}$ of THF. Compound $7 \mathbf{a b}(1.45 \mathrm{~g}, 3.72 \mathrm{mmol}, 83 \%)$ was obtained as a white foam which decomposes at $68{ }^{\circ} \mathrm{C} . \mathrm{R}_{\mathrm{f}}=0.3,70 \%$ ethyl acetate in hexanes; ${ }^{1} \mathrm{H}-\mathrm{NMR}\left(400 \mathrm{MHz}, \mathrm{CDCl}_{3}\right): \delta=7.61(\mathrm{~d}, \mathrm{~J}=6.8 \mathrm{~Hz}, 2 \mathrm{H}), 7.51(\mathrm{~d}, \mathrm{~J}=8.8 \mathrm{~Hz}, 2 \mathrm{H}), 7.42(\mathrm{t}, \mathrm{J}=$ $8.0 \mathrm{~Hz}, 2 \mathrm{H}), 7.32-7.36(\mathrm{~m}, 1 \mathrm{H}), 7.23-7.16(\mathrm{~m}, 3 \mathrm{H}), 6.89(\mathrm{~s}, 4 \mathrm{H}), 5.26(\mathrm{dd}, \mathrm{J}=3.2,12.0 \mathrm{~Hz}, 1 \mathrm{H})$, $4.80(\mathrm{~s}, 1 \mathrm{H}), 4.44(\mathrm{dd}, \mathrm{J}=4.0,10.4 \mathrm{~Hz}, 1 \mathrm{H}), 4.17(\mathrm{~d}, \mathrm{~J}=10.4 \mathrm{~Hz}, 1 \mathrm{H}) 3.20(\mathrm{~s}, 2 \mathrm{H}), 3.10(\mathrm{~s}, 1 \mathrm{H})$, $2.14(\mathrm{~s}, 3 \mathrm{H}), 2.04(\mathrm{~s}, 1 \mathrm{H}), 1.98-1.85(\mathrm{~m}, 2 \mathrm{H}) ;{ }^{13} \mathrm{C}-\mathrm{NMR}\left(100 \mathrm{MHz}, \mathrm{CDCl}_{3}\right): \delta=146.8,140.6$, $136.8,130.8,130.3,128.9,128.5,128.0,127.9,127.4,126.1,115.8,77.4,69.0,66.6,65.0,42.8$, 32.6, 20.5; IR (thin film): $v_{\max }=3377,3031,2923,1616,1509,1453,1307,1240,1031,909$, 815, 735, 700; HRMS calc'd for $\mathrm{C}_{25} \mathrm{H}_{27} \mathrm{O}_{3} \mathrm{~N}=389.1991$, found 389.1996 .

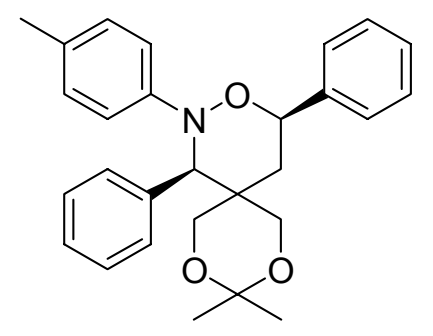

\section{Compound 7ac}

Compound 7ac was prepared from compound 7ab utilizing the general procedure for Step C. Amounts of reagents included $1.45 \mathrm{~g}(3.73 \mathrm{mmol})$ of $7 \mathbf{a b}, 807 \mathrm{mg}(11.2 \mathrm{mmol})$ of 2methoxypropene, and $142 \mathrm{mg}(0.746 \mathrm{mmol})$ of p-toluenesulfonic acid monohydrate in $40 \mathrm{~mL}$ of DMF. Compound 7ac $(1.36 \mathrm{~g}, 3.17 \mathrm{mmol}, 85 \%)$ was obtained as a white powder; $\mathrm{MP}=136$ $138{ }^{\circ} \mathrm{C} ; \mathrm{R}_{\mathrm{f}}=0.6,30 \%$ ethyl acetate in hexanes; ${ }^{1} \mathrm{H}-\mathrm{NMR}\left(400 \mathrm{MHz}, \mathrm{CDCl}_{3}\right): \delta=7.69(\mathrm{~d}, \mathrm{~J}=$ $7.2 \mathrm{~Hz}, 2 \mathrm{H}), 7.50(\mathrm{~d}, \mathrm{~J}=7.2 \mathrm{~Hz}, 2 \mathrm{H}), 7.42(\mathrm{t}, \mathrm{J}=8.4 \mathrm{~Hz}, 2 \mathrm{H}), 7.36-7.32(\mathrm{~m}, 1 \mathrm{H}), 7.22-7.16(\mathrm{~m}$, $3 \mathrm{H}), 6.91(\mathrm{~s}, 4 \mathrm{H}), 5.15(\mathrm{dd}, \mathrm{J}=2.4,12.0 \mathrm{~Hz}, 1 \mathrm{H}), 5.09(\mathrm{~s}, 1 \mathrm{H}), 4.61(\mathrm{dd}, \mathrm{J}=2.0,11.2 \mathrm{~Hz}, 1 \mathrm{H})$, $4.04(\mathrm{~d}, \mathrm{~J}=11.6 \mathrm{~Hz}, 1 \mathrm{H}), 3.35(\mathrm{~d}, \mathrm{~J}=11.6 \mathrm{~Hz}, 1 \mathrm{H}), 3.23(\mathrm{dd}, \mathrm{J}=11.8,1.6 \mathrm{~Hz}, 1 \mathrm{H}), 2.15(\mathrm{~s}$, $3 \mathrm{H}), 1.92(\mathrm{dd}, \mathrm{J}=12.4,12.4 \mathrm{~Hz}, 1 \mathrm{H}), 1.72(\mathrm{dd}, \mathrm{J}=14.0,2.4 \mathrm{~Hz}, 1 \mathrm{H}) 1.54(\mathrm{~s}, 3 \mathrm{H}), 1.47(\mathrm{~s}, 3 \mathrm{H})$; ${ }^{13} \mathrm{C}-\mathrm{NMR}\left(100 \mathrm{MHz}, \mathrm{CDCl}_{3}\right): \delta=146.9,140.1,136.7,130.3,129.9,128.9,128.5,128.0,127.9$, 127.2, 126.2, 115.5, 98.2, 77.3, 66.6, 66.5, 64.8, 36.6, 22.8, 27.3, 20. 4, 20.3; IR (thin film): $v_{\max }=3030,2991,2935,2864,1613,1509,1452,1373,1344,1259,1200,1161,1134,1064$, 1032, 1008, 961, 701; HRMS calc'd for $\mathrm{C}_{28} \mathrm{H}_{31} \mathrm{O}_{3} \mathrm{~N}=429.2304$, found 429.2300. 


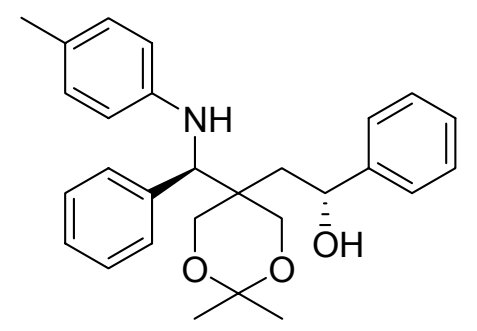

\section{Compound 7ad}

Compound 7ad was obtained using the general procedure for Step D. $500 \mathrm{mg}(1.16 \mathrm{mmol})$ of $7 \mathbf{a c}$ was heated in refluxing THF in the presence of $671.5 \mathrm{mg}(4.47 \mathrm{mmol})$ of $\mathrm{Sm}^{\circ}$ and $1.03 \mathrm{~g}$ $(4.06 \mathrm{mmol}) \mathrm{I}_{2}$ yielding $7 \mathbf{a d}(475 \mathrm{mg}, 1.10 \mathrm{mmol}, 95 \%)$ as a white powder. Note: This amino alcohol was purified for characterization purposes only. In all other examples the crude aminoalcohol was converted to the corresponding pyrrolidine without purification. $\mathrm{MP}=147$ $149^{\circ} \mathrm{C} ; \mathrm{R}_{\mathrm{f}}=0.4,30 \%$ ethyl acetate in hexanes; ${ }^{1} \mathrm{H}-\mathrm{NMR}\left(400 \mathrm{MHz}, \mathrm{CDCl}_{3}\right): \delta=7.39-7.33(\mathrm{~m}$, $6 \mathrm{H}), 7.31-7.25(\mathrm{~m}, 3 \mathrm{H}), 7.21(\mathrm{dd}, \mathrm{J}=7.2,7.2 \mathrm{~Hz}, 1 \mathrm{H}), 6.86(\mathrm{~d}, \mathrm{~J}=8.4 \mathrm{~Hz}, 2 \mathrm{H}), 6.43(\mathrm{~d}, \mathrm{~J}=8.4$ $\mathrm{Hz}, 2 \mathrm{H}), 5.08$ (dd, J = 0.8, $10.6 \mathrm{~Hz}, 1 \mathrm{H}), 4.50(\mathrm{~s}, 1 \mathrm{H}), 4.25$ (dd, J = 0.8, $12.2 \mathrm{~Hz}, 1 \mathrm{H}), 4.09$ (d, J $=12.0 \mathrm{~Hz}, 1 \mathrm{H}) 3.92(\mathrm{~d}, \mathrm{~J}=11.6 \mathrm{~Hz}, 1 \mathrm{H}), 3.39(\mathrm{dd}, \mathrm{J}=12.0,1.2 \mathrm{~Hz}, 1 \mathrm{H}), 2.16(\mathrm{~s}, 3 \mathrm{H}), 1.94(\mathrm{dd}$, $\mathrm{J}=10.8,15.6 \mathrm{~Hz}, 1 \mathrm{H}), 1.75(\mathrm{dd}, \mathrm{J}=15.6,2.0 \mathrm{~Hz}, 1 \mathrm{H}), 1.53(\mathrm{~s}, 3 \mathrm{H}), 1.46(\mathrm{~s}, 3 \mathrm{H}) ;{ }^{13} \mathrm{C}-\mathrm{NMR}$ $\left(100 \mathrm{MHz}, \mathrm{CDCl}_{3}\right): \delta=145.6,145.0,139.3,129.5,128.5,128.5,128.1,127.5,127.2$ 125.9, $125.5,113.4,98.3,71.0,68.0,66.1,61.2,39.6,39.3,26.2,21.5,20.3$; IR (thin film): $v_{\max }=$ 3331, 2992, 2933, 2921, 2871, 1671, 1522, 1452, 1375, 1253, 1199, 1106, 1076, 1058, 1028, $909,808,732,704$; HRMS calc'd for $\mathrm{C}_{28} \mathrm{H}_{33} \mathrm{O}_{3} \mathrm{~N}=431.2460$, found 431.2463

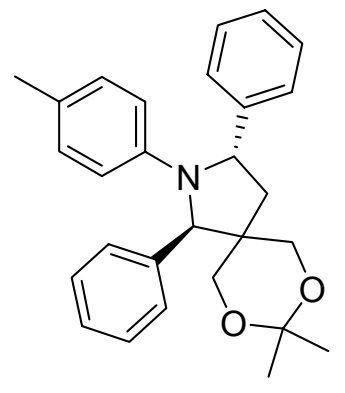

\section{Compound 7a}

Compound 7a was prepared from compound 7ac utilizing the general procedure for Steps D and E. Amounts of reagents for Step D: $100 \mathrm{mg}(0.232 \mathrm{mmol})$ of Compound $7 \mathbf{b c}, 134 \mathrm{mg}(0.893$ $\mathrm{mmol})$ of samarium metal, $203 \mathrm{mg}(0.812 \mathrm{mmol})$ of Iodine. Reagents for Step E: $10.5 \mathrm{mg}(0.350$ $\mathrm{mmol})$ of $\mathrm{NaH}(80 \%$ dispersion in mineral oil), and $35 \mathrm{mg}(0.302 \mathrm{mmol})$ of methanesulfonyl chloride. Compound 7a was obtained (74 $\mathrm{mg}, 0.179 \mathrm{mmol}, 77 \%$ for two steps) as a white powder. $\mathrm{MP}=106-108{ }^{\circ} \mathrm{C} ; \mathrm{R}_{\mathrm{f}}=0.5,30 \%$ ethyl acetate in hexanes; ${ }^{1} \mathrm{H}-\mathrm{NMR}(400 \mathrm{MHz}$, $\left.\mathrm{CDCl}_{3}\right): \delta=7.24-7.22(\mathrm{~m}, 5 \mathrm{H}), 7.15(\mathrm{dd}, \mathrm{J}=7.8,7.8 \mathrm{~Hz}, 2 \mathrm{H}), 6.74(\mathrm{~d}, \mathrm{~J}=8.4 \mathrm{~Hz}, 2 \mathrm{H}), 6.28(\mathrm{~d}, \mathrm{~J}$ $=8.4 \mathrm{~Hz}, 2 \mathrm{H}), 5.74(\mathrm{~s}, 1 \mathrm{H}), 5.19(\mathrm{~d}, \mathrm{~J}=10.2 \mathrm{~Hz}, \mathrm{H}), 4.02(\mathrm{~d}, 11.4 \mathrm{~Hz}, 1 \mathrm{H}), 3.56(\mathrm{~d}, \mathrm{~J}=12.0 \mathrm{~Hz}$, 
$1 \mathrm{H}), 3.32(\mathrm{~d}, \mathrm{~J}=12.0 \mathrm{~Hz}, 1 \mathrm{H}), 2.95(\mathrm{~d}, \mathrm{~J}=12.0 \mathrm{~Hz}, 1 \mathrm{H}), 2.46(\mathrm{dd}, \mathrm{J}=11.4,11.4 \mathrm{~Hz}, 1 \mathrm{H}), 2.07$ $(\mathrm{s}, 3 \mathrm{H}), 1.81(\mathrm{~d}, \mathrm{~J}=12.3 \mathrm{~Hz}, 1 \mathrm{H}), 1.46(\mathrm{~s}, 3 \mathrm{H}), 1.31(\mathrm{~s}, 3 \mathrm{H}) ;{ }^{13} \mathrm{C}-\mathrm{NMR}\left(100 \mathrm{MHz}, \mathrm{CDCl}_{3}\right): \delta=$ $143.9,143.0,140.0,129.0,128.4,128.3,127.0,126.5,126.3,125.1,115.0,97.8,68.6,64.3,61.6$, 45.4, 39.3, 27.6, 20.2, 20.0; *Note 2 Carbon peaks appear to be absent presumably due to overlap; IR (thin film): $v_{\max }=3029,2991,2924,2864,1619,1517,1451,1370,1334,1267$, $1203,1155,1120,1070,1032,981,909,832,803,734,701$; HRMS calc'd for $\mathrm{C}_{28} \mathrm{H}_{31} \mathrm{O}_{2} \mathrm{~N}=$ 413.2355, found 413.2345

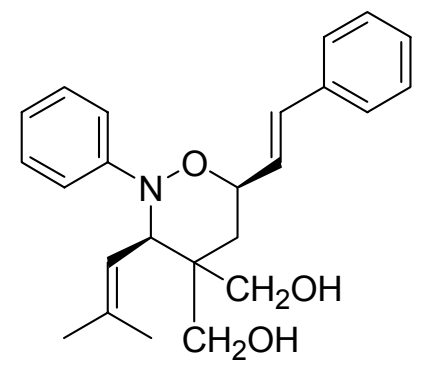

Compound 7bb

Compound $\mathbf{7 b b}$ was prepared from compound $\mathbf{7 b a}$ utilizing the general procedure for Step B. Amounts of reagents included $2.0 \mathrm{~g}(4.59 \mathrm{mmol})$ of $7 \mathrm{ba}$ and $9.8 \mathrm{~mL}(9.8 \mathrm{mmol})$ of $1 \mathrm{M}$ lithium aluminum hydride in $40 \mathrm{~mL}$ of THF. Compound $7 \mathbf{b b}(1.51 \mathrm{~g}, 3.98 \mathrm{mmol}, 87 \%)$ was obtained as a light yellow powder. $\mathrm{MP}=148-150^{\circ} \mathrm{C} ; \mathrm{R}_{\mathrm{f}}=0.2,70 \%$ ethyl acetate in hexanes; ${ }^{1} \mathrm{H}$-NMR (400 $\left.\mathrm{MHz}, \mathrm{CDCl}_{3}\right): \delta=7.43(\mathrm{~d}, \mathrm{~J}=7.2 \mathrm{~Hz}, 2 \mathrm{H}), 7.34(\mathrm{t}, \mathrm{J}=7.6 \mathrm{~Hz}, 2 \mathrm{H}), 7.27(\mathrm{~m}, 4 \mathrm{H}), 7.02(\mathrm{~d}, \mathrm{~J}=$ $8.8 \mathrm{~Hz}, 2 \mathrm{H}), 6.91(\mathrm{t}, \mathrm{J}=7.2 \mathrm{~Hz}, 1 \mathrm{H}), 6.72(\mathrm{~d}, \mathrm{~J}=16.4 \mathrm{~Hz}, \mathrm{H}), 6.26(\mathrm{dd}, \mathrm{J}=6.0,16 \mathrm{~Hz}, 1 \mathrm{H}), 5.61$ $(\mathrm{d}, \mathrm{J}=10.4 \mathrm{~Hz}, 1 \mathrm{H}), 4.79-4.83(\mathrm{~m}, 1 \mathrm{H}), 4.43(\mathrm{~d}, \mathrm{~J}=10.4 \mathrm{~Hz}, 1 \mathrm{H}), 4.34(\mathrm{~d}, \mathrm{~J}=10.4 \mathrm{~Hz}, 1 \mathrm{H})$, $4.11(\mathrm{~d}, \mathrm{~J}=10.8 \mathrm{~Hz}, 1 \mathrm{H}), 3.59$ (dd, J = 14.8, $4.0 \mathrm{~Hz}, 2 \mathrm{H}), 3.07$ (s, 1H), $2.34(\mathrm{~s}, 1 \mathrm{H}), 1.72-1.57$ $(\mathrm{m}, 2 \mathrm{H}), 1.66(\mathrm{~s}, 3 \mathrm{H}), 1.53(\mathrm{~s}, 3 \mathrm{H}) ;{ }^{13} \mathrm{C}-\mathrm{NMR}\left(100 \mathrm{MHz}, \mathrm{CDCl}_{3}\right): \delta=149.6,137.7,136.5$, $131.9,128.5,128.4,127.8,126.5,121.3,116.9,116.2,76.3,69.3,66.7,597,42.2,32.0,26.0$, 18.8 ; Note 1 carbon peak appears to be absent, presumably due to overlap. IR (thin film): $v_{\max }=$ 3375, 2929, 2914, 2883, 1597, 1491, 1449, 1029, 967, 910, 749, 693; HRMS calc'd for $\mathrm{C}_{24} \mathrm{H}_{39} \mathrm{O}_{3} \mathrm{~N}=379.2147$, found 379.2150

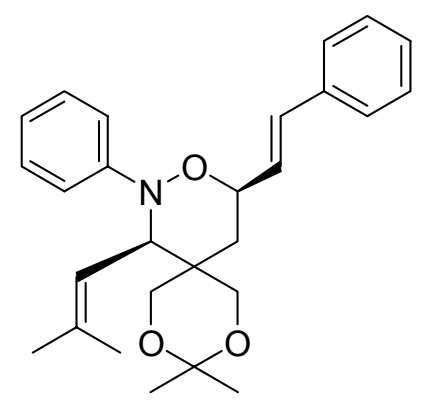

Compound 7bc 
Compound $\mathbf{7 b c}$ was prepared from compound $\mathbf{7 b b}$ utilizing the general procedure for Step C. Amounts of reagents included $1.45 \mathrm{~g}(3.82 \mathrm{mmol})$ of $7 \mathbf{b b}, 824 \mathrm{mg}(11.4 \mathrm{mmol})$ of 2 methoxypropene, and $145 \mathrm{mg}(0.762 \mathrm{mmol})$ of p-toluenesulfonic acid monohydrate in $40 \mathrm{~mL}$ of DMF. Compound $7 \mathbf{b c}(1.53 \mathrm{~g}, 3.65 \mathrm{mmol}, 96 \%)$ was obtained as a colorless foam. $\mathrm{MP}=108$ $111^{\circ} \mathrm{C} ; \mathrm{R}_{\mathrm{f}}=0.6,30 \%$ ethyl acetate in hexanes; ${ }^{1} \mathrm{H}-\mathrm{NMR}\left(400 \mathrm{MHz}, \mathrm{CDCl}_{3}\right): \delta=7.40(\mathrm{~d}, \mathrm{~J}=$ $8.4 \mathrm{~Hz}, 2 \mathrm{H}), 7.32(\mathrm{t}, \mathrm{J}=6.8 \mathrm{~Hz}, 2 \mathrm{H}), 7.26-7.20(\mathrm{~m}, 4 \mathrm{H}) 7.02(\mathrm{~d}, \mathrm{~J}=8.0 \mathrm{~Hz}, 2 \mathrm{H}), 6.87(\mathrm{t}, \mathrm{J}=7.2$ $\mathrm{Hz}, 1 \mathrm{H}), 6.67(\mathrm{~d}, \mathrm{~J}=16.0 \mathrm{~Hz}, 1 \mathrm{H}), 6.22(\mathrm{dd}, \mathrm{J}=6.0,16.0 \mathrm{~Hz}, 1 \mathrm{H}), 5.51(\mathrm{~d}, \mathrm{~J}=10.4 \mathrm{~Hz}, 1 \mathrm{H})$, 4.70-4.65 (m, 1H), $4.64(\mathrm{~d}, \mathrm{~J}=10.4 \mathrm{~Hz}, 1 \mathrm{H}), 4.45(\mathrm{dd}, \mathrm{J}=1.6,11.6 \mathrm{~Hz}, 1 \mathrm{H}), 3.94(\mathrm{~d}, \mathrm{~J}=11.6$ $\mathrm{Hz}, 1 \mathrm{H}), 3.60(\mathrm{dd}, \mathrm{J}=1.6,12.0 \mathrm{~Hz}, 1 \mathrm{H}), 3.51(\mathrm{~d}, \mathrm{~J}=11.6 \mathrm{~Hz}, 1 \mathrm{H}), 1.63(\mathrm{dd}, \mathrm{J}=.2 .0,12.0 \mathrm{~Hz}$, $6 \mathrm{H}), 1.57-1.53(\mathrm{~m}, 2 \mathrm{H}), 1.46(\mathrm{~s}, 6 \mathrm{H}) ;{ }^{13} \mathrm{C}-\mathrm{NMR}\left(100 \mathrm{MHz}, \mathrm{CDCl}_{3}\right): \delta=149.9,138.5,136.5$, 132.0, 128.6, 128.4, 127.9, 127.7, 126.6, 121.1, 116.4, 116.0, 98.2, 75.9, 67.0, 66.4, 60.2, 36.0, 33.4, 26.7, 26.1, 20.9, 18.9; IR (thin film): $v_{\max }=2990,2915,2861,1598,1492,1451,1374$, 1257, 1199, 1069, 1030, 965, 934, 834, 751, 692; HRMS calc'd for $\mathrm{C}_{27} \mathrm{H}_{33} \mathrm{O}_{3} \mathrm{~N}=419.2460$, found 419.2456

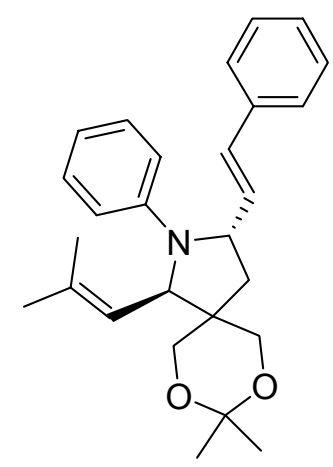

\section{Compound 7b}

Compound $\mathbf{7 b}$ was prepared from compound $\mathbf{7 b c}$ utilizing the general procedure for Steps D and E. Amounts of reagents Step D: $100 \mathrm{mg}(0.239 \mathrm{mmol})$ of Compound $7 \mathbf{b c}, 138 \mathrm{mg}(0.920 \mathrm{mmol})$ of Samarium metal, $212 \mathrm{mg}(0.836 \mathrm{mmol})$ of Iodine. Step E: $11 \mathrm{mg}(0.389 \mathrm{mmol})$ of $\mathrm{NaH}(80 \%$ dispersion in mineral oil), and $36 \mathrm{mg}(0.311 \mathrm{mmol})$ of methanesulfonyl chloride. Compound $\mathbf{7 b}$ (49 $\mathrm{mg}, 0.120 \mathrm{mmol}, 50 \%$ for 2 steps) was obtained as a light orange powder. $\mathrm{MP}=96-99^{\circ} \mathrm{C} ; \mathrm{R}_{\mathrm{f}}$ $=0.5,30 \%$ ethyl acetate in hexanes; ${ }^{1} \mathrm{H}-\mathrm{NMR}\left(400 \mathrm{MHz}, \mathrm{CDCl}_{3}\right): \delta=7.30-7.24(\mathrm{~m}, 5 \mathrm{H}), 7.20-$ $7.18(\mathrm{~m}, 1 \mathrm{H}), 7.12(\mathrm{dt}, \mathrm{J}=2.0,7.2 \mathrm{~Hz}, 2 \mathrm{H}), 6.65-6.59(\mathrm{~m}, 3 \mathrm{H}), 6.46(\mathrm{dd}, \mathrm{J}=1.2,16.0 \mathrm{~Hz}, 1 \mathrm{H})$, $6.16(\mathrm{dd}, \mathrm{J}=5.6,16.0 \mathrm{~Hz}, 1 \mathrm{H}), 4.94(\mathrm{~d}, \mathrm{~J}=10.0 \mathrm{~Hz}, 1 \mathrm{H}), 4.74(\mathrm{~d}, \mathrm{~J}=10.4 \mathrm{~Hz}, 1 \mathrm{H}), 4.42-4.38$ (m, 1H), $4.02(\mathrm{dd}, \mathrm{J}=2.0,11.6 \mathrm{~Hz}, 1 \mathrm{H}), 3.77(\mathrm{~d}, \mathrm{~J}=11.6 \mathrm{~Hz}, 1 \mathrm{H}), 3.76-3.64(\mathrm{~m}, 2 \mathrm{H}), 2.17(\mathrm{dd}, \mathrm{J}$ $=10.0,13.2 \mathrm{~Hz}, 1 \mathrm{H}), 1.90(\mathrm{~d}, \mathrm{~J}=1.2 \mathrm{~Hz}, 3 \mathrm{H}), 1.68(\mathrm{~d}, \mathrm{~J}=1.2 \mathrm{~Hz}, 3 \mathrm{H}), 1.71-1.68(\mathrm{~m}, 1 \mathrm{H}), 1.41$ (s, $6 \mathrm{H}) ;{ }^{13} \mathrm{C}-\mathrm{NMR}\left(100 \mathrm{MHz}, \mathrm{CDCl}_{3}\right): \delta=146.5,137.2,136.8,133.8,129.9,128.5,128.5,127.3$, 126.3, 121.2, 116.2, 114.7, 97.9, 68.3, 64.7, 62.2, 58.1, 43.9, 38.0, 27.3, 25.9, 20.4, 18.4; IR (thin film): $v_{\max }=3026,2990,2967,2935,2862,1597,1500,1450,1372,1340,1258,1202,1068$, 1034, 965, 909, 835, 806, 748, 732, 692; HRMS calc'd for $\mathrm{C}_{27} \mathrm{H}_{33} \mathrm{O}_{2} \mathrm{~N}=403.2511$, found 403.2518; 


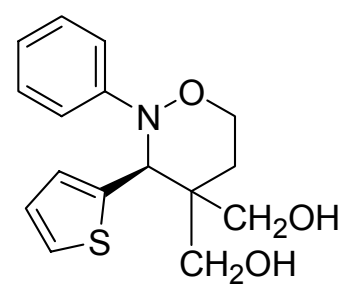

\section{Compound $7 \mathrm{cb}$}

Compound 7cb was prepared from compound 7ca utilizing the general procedure for Step B. Amounts of reagents included $2.3 \mathrm{~g}(6.37 \mathrm{mmol})$ of $7 \mathbf{c a}$ and $15.9 \mathrm{ml}(15.9 \mathrm{mmol})$ of $1 \mathrm{M}$ lithium aluminum hydride in $40 \mathrm{~mL}$ of THF. Compound $7 \mathbf{c b}(1.83 \mathrm{~g}, 5.99 \mathrm{mmol}, 94 \%)$ was obtained as a pale brown powder. $\mathrm{MP}=118-119^{\circ} \mathrm{C} ; \mathrm{R}_{\mathrm{f}}=0.2,70 \%$ ethyl acetate in hexanes; ${ }^{1} \mathrm{H}-\mathrm{NMR}(400$ $\left.\mathrm{MHz}, \mathrm{CDCl}_{3}\right): \delta=7.16-7.12(\mathrm{~m}, 3 \mathrm{H}), 6.98(\mathrm{dd}, \mathrm{J}=1.2,8.8 \mathrm{~Hz}, 2 \mathrm{H}), 6.93(\mathrm{dd}, \mathrm{J}=1.2,3.6 \mathrm{~Hz}$, $1 \mathrm{H}), 6.83-6.80(\mathrm{~m}, 2 \mathrm{H}), 5.28(\mathrm{~s}, 1 \mathrm{H}), 4.40(\mathrm{~d}, \mathrm{~J}=10.0 \mathrm{~Hz}, 1 \mathrm{H}), 4.27(\mathrm{dt}, \mathrm{J}=2.8,11.0 \mathrm{~Hz}, 1 \mathrm{H})$, $4.19(\mathrm{dd}, \mathrm{J}=6.8,12.0 \mathrm{~Hz}, 1 \mathrm{H}), 4.05(\mathrm{~d}, \mathrm{~J}=10.8 \mathrm{~Hz}, 1 \mathrm{H}), 3.36(\mathrm{~d}, \mathrm{~J}=10.4 \mathrm{~Hz}, 1 \mathrm{H}), 3.26(\mathrm{~d}, \mathrm{~J}=$ $10.4 \mathrm{~Hz}, \mathrm{H}), 2.94(\mathrm{~s}, 1 \mathrm{H}), 2.10(\mathrm{~s}, 1 \mathrm{H}), 1.98(\mathrm{dt}, \mathrm{J}=6.0,12.8 \mathrm{~Hz}, 1 \mathrm{H}), 1.39$ (dd, J = 2.8, 14.4 $\mathrm{Hz}, 1 \mathrm{H}) ;{ }^{13} \mathrm{C}-\mathrm{NMR}\left(100 \mathrm{MHz}, \mathrm{CDCl}_{3}\right): \delta=149.0,136.0,128.4,128.4,126.1,125.3,121.6$, $115.9,68.7,66.5,65.6,62.7,41.5,25.4$; IR (thin film): $v_{\max }=3384,2942,1597,1491,1368$, $1317,1248,1153,1109,1052,909,853,757,695,649$; HRMS calc'd for $\mathrm{C}_{16} \mathrm{H}_{19} \mathrm{O}_{3} \mathrm{NS}=$ 305.1086 , found 305.1086

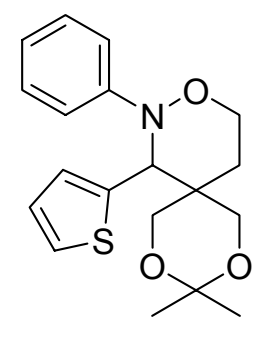

\section{Compound 7cc}

Compound 7cc was prepared from compound 7cb utilizing the general procedure for Step C. Amounts of reagents included $500 \mathrm{mg}(1.64 \mathrm{mmol})$ of $7 \mathrm{cb}, 354 \mathrm{mg}$ (4.91 mmol) of 2methoxypropene, and $62 \mathrm{mg}(0.326 \mathrm{mmol})$ of p-toluenesulfonic acid monohydrate in $20 \mathrm{~mL}$ of DMF. Compound 7cc (486 mg, $1.41 \mathrm{mmol}, 86 \%$ ) was obtained as a white foam. MP = 85$86^{\circ} \mathrm{C} ; \mathrm{R}_{\mathrm{f}}=0.6,30 \%$ ethyl acetate in hexanes; ${ }^{1} \mathrm{H}-\mathrm{NMR}\left(400 \mathrm{MHz}, \mathrm{CDCl}_{3}\right): \delta=7.20-7.13(\mathrm{~m}$, $3 \mathrm{H})$, 7.04-7.00 (m, 3H), 6.87-6.83 (m, 2H), $5.45(\mathrm{~s}, 1 \mathrm{H}), 4.53(\mathrm{dd}, \mathrm{J}=2.4,12.0 \mathrm{~Hz}, 2 \mathrm{H}), 4.19$ $(\mathrm{dd}, \mathrm{J}=1.2,8.4 \mathrm{~Hz}, 2 \mathrm{H}), 3.97(\mathrm{~d}, \mathrm{~J}=11.0 \mathrm{~Hz}, 2 \mathrm{H}), 3.39(\mathrm{~d}, \mathrm{~J}=12.0 \mathrm{~Hz}, 1 \mathrm{H}), 3.25(\mathrm{~d}, \mathrm{~J}=2.4 \mathrm{~Hz}$, $12.0 \mathrm{~Hz}, 1 \mathrm{H}), 2.05-1.97(\mathrm{~m}, 1 \mathrm{H}), 1.53(\mathrm{~s}, 3 \mathrm{H}), 1.48(\mathrm{~s}, 3 \mathrm{H}), 1.39$ (d, J = 14.4 Hz, 1H); ${ }^{13} \mathrm{C}-\mathrm{NMR}$ $\left(100 \mathrm{MHz}, \mathrm{CDCl}_{3}\right): \delta=149.1,135.5,128.8,128.4,126.0,125.5,121.6,115.8,98.4,66.6,66.2$, 65.9, 63.5, 35.4, 27.1, 26.9, 20.6; IR (thin film): $v_{\max }=2989,2936,2865,1597,1492,1451$, 1372, 1259, 1198, 1153, 1133, 1072, 1051, 1038, 974, 832, 750, 694, HRMS calc'd for $\mathrm{C}_{19} \mathrm{H}_{23} \mathrm{O}_{3} \mathrm{NS}=345.1399$, found 345.1402 


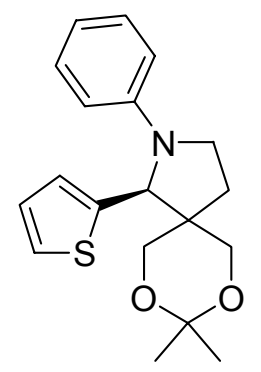

\section{Compound 7c}

Compound 7c was prepared from compound 7cc utilizing the general procedure for Steps D and E. Amounts of reagents Step D: $200 \mathrm{mg}(0.578 \mathrm{mmol})$ of Compound 7cc, $335 \mathrm{mg}(2.22 \mathrm{mmol})$ of Samarium metal, $513 \mathrm{mg}(2.02 \mathrm{mmol})$ of Iodine Step E: $26 \mathrm{mg}(0.867 \mathrm{mmol})$ of $\mathrm{NaH}(80 \%$ dispersion in mineral oil), and $86 \mathrm{mg}(0.751 \mathrm{mmol})$ of methanesulfonyl chloride. Compound 7c (72mg, $0.220 \mathrm{mmol}, 38 \%$ two steps) was obtained from 7cc as colorless cubes; MP $=112-114$ ${ }^{\circ} \mathrm{C} ; \mathrm{R}_{\mathrm{f}}=0.4,30 \%$ ethyl acetate in hexanes. ${ }^{1} \mathrm{H}-\mathrm{NMR}\left(600 \mathrm{MHz}, \mathrm{CDCl}_{3}\right): \delta=7.16-7.13(\mathrm{~m}$, $3 \mathrm{H}), 7.02(\mathrm{~d}, \mathrm{~J}=3.6 \mathrm{~Hz}, 1 \mathrm{H}), 6.96(\mathrm{dd}, \mathrm{J}=3.0,4.2 \mathrm{~Hz}, 1 \mathrm{H}), 6.66(\mathrm{t}, \mathrm{J}=8.4 \mathrm{~Hz}, 1 \mathrm{H}), 6.57(\mathrm{~d}, \mathrm{~J}=$ $7.8 \mathrm{~Hz}, 2 \mathrm{H}), 5.09(\mathrm{~s}, 1 \mathrm{H}), 3.76(\mathrm{~d}, \mathrm{~J}=11.4 \mathrm{~Hz}, 1 \mathrm{H}), 3.70(\mathrm{dd}, \mathrm{J}=1.8,12.0 \mathrm{~Hz}, 1 \mathrm{H}), 3.64(\mathrm{dt}, \mathrm{J}=$ $1.8,9.6 \mathrm{~Hz}, 1 \mathrm{H}), 3.45(\mathrm{~d}, \mathrm{~J}=12.0 \mathrm{~Hz}, 1 \mathrm{H}), 3.42-3.39(\mathrm{~m}, 2 \mathrm{H}), 2.05-2.00(\mathrm{~m}, 1 \mathrm{H}), 1.94(\mathrm{dd}, \mathrm{J}=$ 6.0, $12.0 \mathrm{~Hz}, 1 \mathrm{H}), 1.48(\mathrm{~s}, 3 \mathrm{H}), 1.44(\mathrm{~s}, 3 \mathrm{H}) ;{ }^{13} \mathrm{C}-\mathrm{NMR}\left(100 \mathrm{MHz}, \mathrm{CDCl}_{3}\right): \delta=146.5,145.2$, 129.0, 127.1, 125.0, 124.1, 116.5, 112.1, 98.2, 67.0, 64.5, 62.9, 45.5, 45.4, 28.5, 25.9, 21.7; IR (thin film): $v_{\max }=2991,2954,2929,2863,1598,1504,1453,1371,1359,1252,1220,1200$, 1154, 1120, 1072, 1036, 1016, 999, 832, 749, 693; HRMS calc'd for $\mathrm{C}_{19} \mathrm{H}_{23} \mathrm{O}_{2} \mathrm{NS}=329.1449$, found 329.1441

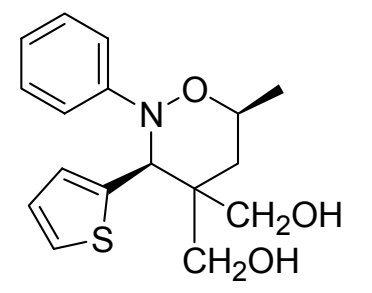

\section{Compound 7db}

Compound 7db was prepared from compound 7da utilizing the general procedure for Step B. Amounts of reagents included $1.5 \mathrm{~g}(4.00 \mathrm{mmol})$ of $7 \mathbf{d a}$ and $10.0 \mathrm{~mL}(10.0 \mathrm{mmol})$ of $1 \mathrm{M}$ lithium aluminum hydride in $30 \mathrm{~mL}$ of THF. Compound $7 \mathbf{d b}(1.08 \mathrm{~g}, 3.4 \mathrm{mmol}, 85 \%)$ was obtained as a pale brown powder. $\mathrm{MP}=158-159{ }^{\circ} \mathrm{C} ; \mathrm{R}_{\mathrm{f}}=0.2,70 \%$ ethyl acetate in hexanes; ${ }^{1} \mathrm{H}-\mathrm{NMR}(400$ $\left.\mathrm{MHz}, \mathrm{CDCl}_{3}\right): \delta=7.16-7.12(\mathrm{~m}, 3 \mathrm{H}), 7.02-6.99(\mathrm{~m}, 2 \mathrm{H}), 6.93(\mathrm{dd}, \mathrm{J}=1.2,3.4 \mathrm{~Hz}, 1 \mathrm{H}), 6.83-6.78$ $(\mathrm{m}, 2 \mathrm{H}), 5.28(\mathrm{~s}, \mathrm{H}), 4.39(\mathrm{~d}, \mathrm{~J}=10.8 \mathrm{~Hz}, 1 \mathrm{H}), 4.35-4.30(\mathrm{~m}, 1 \mathrm{H}), 4.04(\mathrm{~d}, \mathrm{~J}=10.4 \mathrm{~Hz}, 1 \mathrm{H}), 3.36$ $(\mathrm{d}, \mathrm{J}=10.4 \mathrm{~Hz}, 1 \mathrm{H}), 3.26(\mathrm{~d}, \mathrm{~J}=10.8 \mathrm{~Hz}, \mathrm{H}), 2.79(\mathrm{~s}, 1 \mathrm{H}), 1.88(\mathrm{~s}, 1 \mathrm{H}), 1.57$ (dd, J= 11.6, 14.0 $\mathrm{Hz}, 1 \mathrm{H}), 1.45(\mathrm{dd}, 2.8,14.4 \mathrm{~Hz}, 1 \mathrm{H}), 1.36(\mathrm{~d}, \mathrm{~J}=6.4 \mathrm{~Hz}, 3 \mathrm{H}) ;{ }^{13} \mathrm{C}-\mathrm{NMR}\left(100 \mathrm{MHz}, \mathrm{CDCl}_{3}\right): \delta=$ $149.0,136.1,128.4,128.2,126.2,125.4,121.5,115.9,71.9,69.1,66.6,62.0,42.7,33.1,20.4$; 
IR (thin film): $v_{\max }=3382,3362,2971,2898,1491,1451,1032,904,695$; HRMS calc'd for $\mathrm{C}_{17} \mathrm{H}_{21} \mathrm{O}_{3} \mathrm{NS}=319.1242$, found 319.1242<smiles>CC1CC2(COC(C)(C)OC2)C(c2cccs2)N(c2ccccc2)O1</smiles>

\section{Compound 7dc}

Compound 7dc was prepared from compound $\mathbf{7 d b}$ utilizing the general procedure for Step C. Amounts of reagents included $307 \mathrm{mg}(0.96 \mathrm{mmol})$ of $7 \mathbf{d b}, 211 \mathrm{mg}(2.92 \mathrm{mmol})$ of 2methoxypropene, and $37 \mathrm{mg}(0.195 \mathrm{mmol})$ of p-toluenesulfonic acid monohydrate in $15 \mathrm{~mL}$ of DMF. Compound 7dc (274 mg, $0.763 \mathrm{mmol}, 79 \%$ ) was obtained as a pale yellow powder. MP $=128-130^{\circ} \mathrm{C} ; \mathrm{R}_{\mathrm{f}}=0.4,30 \%$ ethyl acetate in hexanes; ${ }^{1} \mathrm{H}-\mathrm{NMR}\left(400 \mathrm{MHz}, \mathrm{CDCl}_{3}\right): \delta=7.15$ $(\mathrm{dd}, \mathrm{J}=7.2,5.6 \mathrm{~Hz}, 2 \mathrm{H}), 7.11(\mathrm{~d}, \mathrm{~J}=5.2 \mathrm{~Hz}, 1 \mathrm{H}), 7.03(\mathrm{~d}, \mathrm{~J}=7.6,2 \mathrm{H}), 6.97(\mathrm{dd}, \mathrm{J}=1.2,4.0 \mathrm{~Hz}$, $1 \mathrm{H}), 6.85-6.79(\mathrm{~m}, 2 \mathrm{H}), 5.40(\mathrm{~s}, 1 \mathrm{H}), 4.47(\mathrm{dd}, \mathrm{J}=2.4,11.6 \mathrm{~Hz}, 1 \mathrm{H}), 4.26-4.20(\mathrm{~m}, 1 \mathrm{H}) 3.93(\mathrm{~d}$, $\mathrm{J}=12.0 \mathrm{~Hz}, 1 \mathrm{H}), 3.35(\mathrm{~d}, \mathrm{~J}=12.0 \mathrm{~Hz}, 1 \mathrm{H}), 3.19(\mathrm{dd}, \mathrm{J}=2.0,11.8 \mathrm{~Hz}, 1 \mathrm{H}), 1.57(\mathrm{dd}, \mathrm{J}=11.6$, $14.0 \mathrm{~Hz}, 1 \mathrm{H}), 1.57(\mathrm{~s}, 3 \mathrm{H}), 1.47-1.42(\mathrm{~m}, 1 \mathrm{H}), 1.45(\mathrm{~s}, 3 \mathrm{H}), 1.34(\mathrm{~d}, \mathrm{~J}=6.0 \mathrm{~Hz}, 3 \mathrm{H}) ;{ }^{13} \mathrm{C}-\mathrm{NMR}$ $\left(100 \mathrm{MHz}, \mathrm{CDCl}_{3}\right): \delta=149.1,135.5,128.7,128.4,126.0,125.5,121.4,115.8,98.3,71.6,66.5$, $66.3,62.6,36.5,34.4,27.2,20.5,20.1$; IR (thin film): $v_{\max }=3068,2988,2926,2862,1597$, 1492, 1451, 1374, 1342, 1259, 1199, 1154, 1073, 1032, 957, 913, 836, 755, 694, 655, 607; HRMS calc'd for $\mathrm{C}_{20} \mathrm{H}_{25} \mathrm{O}_{3} \mathrm{NS}=359.1555$, found 359.1565

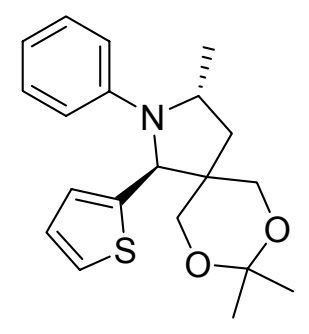

\section{Compound 7d}

Compound 7d was prepared from compound 7de utilizing the general procedure for Steps D and E. Amounts of reagents Step D: $200 \mathrm{mg}(0.557 \mathrm{mmol})$ of Compound 7dc, $322 \mathrm{mg}(2.14 \mathrm{mmol})$ of Samarium metal, $495 \mathrm{mg}(1.95 \mathrm{mmol})$ of Iodine Step E: $25 \mathrm{mg}(0.836 \mathrm{mmol})$ of $\mathrm{NaH}(80 \%$ dispersion in mineral oil), and $83 \mathrm{mg}(0.724 \mathrm{mmol})$ of methanesulfonyl chloride. Compound $7 \mathbf{d}$ was obtained in $54 \%$ yield $(77 \mathrm{mg}, 0.225 \mathrm{mmol}$ ) based on the recovered starting material or $40 \%$ overall $\left(49.9 \mathrm{mg}, 0.139 \mathrm{mmol}\right.$ ) from $7 \mathbf{d c}$ as colorless needles $\mathrm{MP}=108-110^{\circ} \mathrm{C}$. Note the yield of this reaction did not increase when complete consumption of starting material was obtained. Reduction of the thiophene is believed to be a competing process with reductive cleavage of the nitrogen oxygen bond leading to the decreased yield. $R_{f}=0.4,30 \%$ ethyl acetate in hexanes; 
${ }^{1} \mathrm{H}-\mathrm{NMR}\left(400 \mathrm{MHz}, \mathrm{CDCl}_{3}\right): \delta=7.13-7.09(\mathrm{~m}, 2 \mathrm{H}), 7.05(\mathrm{dd}, \mathrm{J}=1.2,5.2 \mathrm{~Hz}, 2 \mathrm{H}), 6.89-6.88$ $(\mathrm{m}, 1 \mathrm{H}) 6.87-6.84(\mathrm{~m}, 1 \mathrm{H}), 6.65(\mathrm{~d}, \mathrm{~J}=4.0 \mathrm{~Hz}, 2 \mathrm{H}), 6.60(\mathrm{dt}, \mathrm{J}=1.2,7.6 \mathrm{~Hz}, 1 \mathrm{H}), 5.56(\mathrm{~s}, 1 \mathrm{H})$, 4.19-4.15 (m, 1H), $4.06(\mathrm{dd}, \mathrm{J}=2.0,11.2 \mathrm{~Hz}, 1 \mathrm{H}), 3.94(\mathrm{~d}, \mathrm{~J}=11.2 \mathrm{~Hz}, 1 \mathrm{H}), 3.51(\mathrm{~d}, \mathrm{~J}=12 \mathrm{~Hz}$, $1 \mathrm{H}), 3.17(\mathrm{dd}, \mathrm{J}=2.4,11.8 \mathrm{~Hz}, 1 \mathrm{H}), 2.21(\mathrm{dd}, \mathrm{J}=9.6,13.6 \mathrm{~Hz}, 1 \mathrm{H}), 1.48(\mathrm{~s}, 3 \mathrm{H}), 1.45(\mathrm{~s}, 3 \mathrm{H})$, $1.51-1.42(\mathrm{~m}, 1 \mathrm{H}), 1.31(\mathrm{~d}, \mathrm{~J}=6.0 \mathrm{~Hz}, 3 \mathrm{H}) ;{ }^{13} \mathrm{C}-\mathrm{NMR}\left(100 \mathrm{MHz}, \mathrm{CDCl}_{3}\right): \delta=145.5,143.9,128.8$, 126.6, 126.4, 124.1, 116.6, 114.8, 97.9, 68.7, 64.5, 64.0, 51.9, 48.9, 37.1, 27.5, 21.6, 20.2; IR (thin film): $v_{\max }=2989,2970,2932,2864,1598,1501,1454,1374,1337,1255,1204,1175$, $1155,1071,1038,834,748,693$; HRMS calc'd for $\mathrm{C}_{20} \mathrm{H}_{25} \mathrm{O}_{2} \mathrm{NS}=343.1606$, found 343.1602<smiles>CN1O[C@@H](c2ccccc2)CC(C)(CO)[C@H]1c1ccccc1</smiles>

\section{Compound 7eb}

Compound 7eb was prepared from compound 7ea utilizing the general procedure for Step B. Amounts of reagents included $2.3 \mathrm{~g}(6.21 \mathrm{mmol})$ of 7 ea and $15.5 \mathrm{~mL}(15.5 \mathrm{mmol})$ of $1 \mathrm{M}$ lithium aluminum hydride in $50 \mathrm{~mL}$ of THF. Compound $7 \mathbf{e b}(1.36 \mathrm{~g}, 4.35 \mathrm{mmol}, 70 \%)$ was obtained as a colorless foam; $\mathrm{MP}=138-140^{\circ} \mathrm{C} ; \mathrm{R}_{\mathrm{f}}=0.2,70 \%$ ethyl acetate in hexanes; ${ }^{1} \mathrm{H}-\mathrm{NMR}(400 \mathrm{MHz}$, $\left.\mathrm{CDCl}_{3}\right): \delta=8.08(\mathrm{~s}, 1 \mathrm{H}), 7.44(\mathrm{~d}, \mathrm{~J}=2.4 \mathrm{~Hz}, 2 \mathrm{H}), 7.40(\mathrm{t}, \mathrm{J}=5.2 \mathrm{~Hz}, 3 \mathrm{H}), 7.34-7.31(\mathrm{~m}, 3 \mathrm{H})$, $7.20(\mathrm{~s}, 1 \mathrm{H}), 5.22$ (dd, J = 2.4, $8.0 \mathrm{~Hz}, 1 \mathrm{H}), 4.32(\mathrm{~d}, \mathrm{~J}=7.2 \mathrm{~Hz}, 1 \mathrm{H}), 4.17(\mathrm{~d}, \mathrm{~J}=7.2 \mathrm{~Hz}, 1 \mathrm{H})$, $3.91(\mathrm{~s}, 1 \mathrm{H}), 3.57(\mathrm{~s}, 1 \mathrm{H}), 3.21(\mathrm{~s}, 2 \mathrm{H}), 2.45(\mathrm{~s}, 3 \mathrm{H}), 1.97(\mathrm{~s}, 1 \mathrm{H}), 1.88-1.78(\mathrm{~m}, 2 \mathrm{H}) ;{ }^{13} \mathrm{C}-\mathrm{NMR}$ $\left(100 \mathrm{MHz}, \mathrm{CDCl}_{3}\right): \delta=141.1,136.2,131.8,129.7,128.5,128.2,127.8,127.7,126.0,77.1,69.0$, 68.4, 68.0, 43.3, 42.6, 32.7, IR (thin film): $v_{\max }=3386,3374,3090,3061,3030,2951,2930$, 2886, 1494, 1454, 1435, 1060, 1032, 912, 891; HRMS calc'd for $\mathrm{C}_{19} \mathrm{H}_{23} \mathrm{O}_{3} \mathrm{~N}=313.1678$, found 313.1676 .

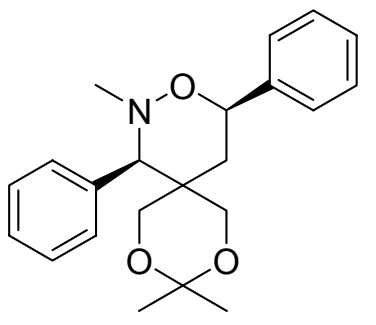

\section{Compound 7ec}

Compound 7ec was prepared from compound 7eb utilizing the general procedure for Step C. Amounts of reagents included $500 \mathrm{mg}(1.64 \mathrm{mmol})$ of $7 \mathrm{eb}, 354 \mathrm{mg}(4.91 \mathrm{mmol})$ of 2methoxypropene, and $62 \mathrm{mg}(0.326 \mathrm{mmol})$ of p-toluenesulfonic acid monohydrate in $20 \mathrm{~mL}$ of DMF. Compound 7ec (486 mg, 1.41mmol, $93 \%$ ) was obtained as a foam oil. $R_{f}=0.6,30 \%$ ethyl acetate in hexanes; ${ }^{1} \mathrm{H}-\mathrm{NMR}\left(400 \mathrm{MHz}, \mathrm{CDCl}_{3}\right): \delta=8.08(\mathrm{~s}, 1 \mathrm{H}), 7.44-7.24(\mathrm{~m}, 9 \mathrm{H}), 5.03$ 
$(\mathrm{dd}, \mathrm{J}=2.8,12.4 \mathrm{~Hz}, 1 \mathrm{H}), 4.57(\mathrm{~d}, \mathrm{~J}=11.2 \mathrm{~Hz}, 1 \mathrm{H}), 4.10(\mathrm{~s}, \mathrm{H}), 4.02(\mathrm{~d}, \mathrm{~J}=11.6 \mathrm{~Hz}, 1 \mathrm{H}), 3.24$ $(\mathrm{d}, \mathrm{J}=12.0 \mathrm{~Hz}, 1 \mathrm{H}), 3.15(\mathrm{~d}, \mathrm{~J}=12.0 \mathrm{~Hz}, 1 \mathrm{H}), 2.51(\mathrm{~s}, 3 \mathrm{H}), 1.82(\mathrm{t}, \mathrm{J}=13.6 \mathrm{~Hz}, \mathrm{H}), 1.66(\mathrm{~d}, \mathrm{~J}=$ $14.0 \mathrm{~Hz}, 1 \mathrm{H}), 1.48(\mathrm{~s}, 3 \mathrm{H}), 1.43(\mathrm{~s}, 3 \mathrm{H}) ;{ }^{13} \mathrm{C}-\mathrm{NMR}\left(100 \mathrm{MHz}, \mathrm{CDCl}_{3}\right): \delta=140.9,136.3,132.2$, 129.3, 128.5, 128.1, 127.9, 127.5, 126.2, 98.1, 76.5, 68.4, 67.0, 66.9, 66.5, 43.5, 36.6, 33.4, 26.5, 21.2; IR (thin film): $v_{\max }=2990,2955,2930,2881,2861,1493,1452,1435,1372,1259,1201$, $1180,1142,1088,1059,1007,935,889,832,752,700$; HRMS calc'd for $\mathrm{C}_{22} \mathrm{H}_{27} \mathrm{O}_{3} \mathrm{~N}=$ 353.1991 , found 353.1990

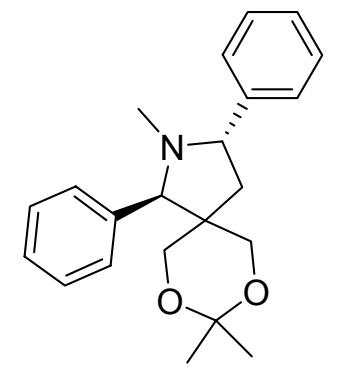

\section{Compound 7e}

Compound 7e was prepared from compound 7ec utilizing the general procedure for Steps D and E. Amounts of reagents Step D: $100 \mathrm{mg}(0.283 \mathrm{mmol})$ of Compound $7 \mathrm{ec}, 164 \mathrm{mg}(1.09 \mathrm{mmol})$ of Samarium metal, $248 \mathrm{mg}(0.99 \mathrm{mmol})$ of Iodine Step E: $13 \mathrm{mg}(0.424 \mathrm{mmol})$ of $\mathrm{NaH}(80 \%$ dispersion in mineral oil), and $42 \mathrm{mg}(0.368 \mathrm{mmol})$ of methanesulfonyl chloride. Compound $7 \mathrm{e}$ (75 mg, $0.22 \mathrm{mmol}, 78 \%$ two steps) was obtained from 7ec as colorless needles. MP $=112-114$ ${ }^{\circ} \mathrm{C} . \quad \mathrm{R}_{\mathrm{f}}=0.4,30 \%$ ethyl acetate in hexanes; ${ }^{1} \mathrm{H}-\mathrm{NMR}\left(400 \mathrm{MHz}, \mathrm{CDCl}_{3}\right): \delta=7.38-7.20(\mathrm{~m}$, $10 \mathrm{H}), 4.32(\mathrm{~s}, 1 \mathrm{H}), 4.33-4.29(\mathrm{~m}, 1 \mathrm{H}), 4.17(\mathrm{dd}, \mathrm{J}=1.6,11.0 \mathrm{~Hz}, 1 \mathrm{H}), 3.79(\mathrm{~d}, \mathrm{~J}=10.8 \mathrm{~Hz}, 1 \mathrm{H})$, $3.46(\mathrm{~d}, \mathrm{~J}=11.6 \mathrm{~Hz}, 1 \mathrm{H}), 3.28$ (dd, J = 1.6, $11.8 \mathrm{~Hz}, 1 \mathrm{H}), 2.47$ (dd, J = 10.0, $14.0 \mathrm{~Hz}, 1 \mathrm{H}), 2.03$ $(\mathrm{s}, 3 \mathrm{H}), 1.78(\mathrm{dd}, \mathrm{J}=5.2,14.0 \mathrm{~Hz}, 1 \mathrm{H}), 1.35(\mathrm{~s}, 6 \mathrm{H}) ;{ }^{13} \mathrm{C}-\mathrm{NMR}\left(100 \mathrm{MHz}, \mathrm{CDCl}_{3}\right): \delta=144.7$, $139.1,128.4,128.2,127.1,127.1,126.8,97.6,73.9,69.6,66.5,66.4,44.2,41.5,35.4,24.9,22.6$ IR (thin film): $v_{\max }=2990,2933,2858,1452,1379,1371,1227,1198,1110,1067,1037,834$, 753, 701; HRMS calc'd for $\mathrm{C}_{22} \mathrm{H}_{27} \mathrm{O}_{2} \mathrm{~N}=337.2042$, found 337.2048; Relative stereochemistry confirmed by X-ray crystallography.<smiles>COC(=O)C1(C(OC)OC)C[C@@H](c2ccco2)ON(C)[C@H]1c1ccccc1</smiles>

\section{Compound 7 fa}

Compound 7fa was prepared from methyl nitrone and furan cyclopropane diester utilizing the general procedure for Step A. Amounts of reagents included $500 \mathrm{mg}(3.69 \mathrm{mmol})$ of methyl nitrone, $689 \mathrm{mg}$ ( $3.08 \mathrm{mmol})$ furan cyclopropane diester, and $190 \mathrm{mg}(0.308 \mathrm{mmol})$ of ytterbium 
triflate, and $40 \mathrm{~mL}$ of $\mathrm{CH}_{2} \mathrm{Cl}_{2}$. Compound $7 \mathbf{f a}(801 \mathrm{mg}, 2.23 \mathrm{mmol}, 73 \%)$ was obtained as a colorless foam oil; $\mathrm{R}_{\mathrm{f}}=0.6,30 \%$ ethyl acetate in hexanes; ${ }^{1} \mathrm{H}-\mathrm{NMR}\left(400 \mathrm{MHz}, \mathrm{CDCl}_{3}\right)$ : $\delta=$ $7.64(\mathrm{~s}, 2 \mathrm{H}), 7.50-7.49(\mathrm{~m}, 1 \mathrm{H}), 7.35-7.33(\mathrm{~m}, 3 \mathrm{H}), 6.52(\mathrm{~d}, \mathrm{~J}=3.2 \mathrm{~Hz}, 2 \mathrm{H}), 6.43(\mathrm{dd}, \mathrm{J}=1.6,3.2$ $\mathrm{Hz}, 1 \mathrm{H}), 4.97(\mathrm{dd}, \mathrm{J}=2.0,12.4 \mathrm{~Hz}, 1 \mathrm{H}), 4.99(\mathrm{dd}, \mathrm{J}=2.0,12.4 \mathrm{~Hz}, 1 \mathrm{H}), 4.83(\mathrm{~s}, \mathrm{H}), 3.87$ (s, 3H), $3.43(\mathrm{~s}, 3 \mathrm{H}), 2.99(\mathrm{dd}, \mathrm{J}=12.4,14.4 \mathrm{~Hz}, 1 \mathrm{H}), 2.61(\mathrm{dd}, \mathrm{J}=2.4,14.41 \mathrm{~Hz}), 2.52(\mathrm{~s}, 3 \mathrm{H}) ;{ }^{13} \mathrm{C}-$ NMR $\left(100 \mathrm{MHz}, \mathrm{CDCl}_{3}\right): \delta=170.3,168.5,152.2,143.2,134.4,131.4,128.5,128.3,110.5$, 108.7, 71.6, 68.1, 59.2, 53.6, 52.7, 43.6, 27.8; IR (thin film): $v_{\max }=2955,2924,2888,2853$, $1742,1453,1435,1363,1310,1262,1196,1158,1097,1065,915,862,743$, 703; HRMS calc'd for $\mathrm{C}_{19} \mathrm{H}_{21} \mathrm{O}_{6} \mathrm{~N}=307.1420$, found 307.1413<smiles>CN1O[C@@H](c2ccco2)CC(C)(CO)[C@H]1c1ccccc1</smiles>

\section{Compound 7fb}

Compound $\mathbf{7 f b}$ was prepared from compound $\mathbf{7 f a}$ utilizing the general procedure for Step B. Amounts of reagents included $1.05 \mathrm{~g}(2.92 \mathrm{mmol})$ of $7 \mathbf{f a}$ and $6.0 \mathrm{~mL}(6.0 \mathrm{mmol})$ of $1 \mathrm{M}$ lithium aluminum hydride in $25 \mathrm{~mL}$ of THF. Compound $7 \mathrm{fb}(649 \mathrm{mg}, 2.14 \mathrm{mmol}, 73 \%)$ was obtained as a pale orange foam. $\mathrm{MP}=118-120{ }^{\circ} \mathrm{C} . \mathrm{R}_{\mathrm{f}}=0.2,70 \%$ ethyl acetate in hexanes; ${ }^{1} \mathrm{H}-\mathrm{NMR}(400$ $\left.\mathrm{MHz}, \mathrm{CDCl}_{3}\right): \delta=8.05(\mathrm{~d}, \mathrm{~J}=6.8 \mathrm{~Hz}, 1 \mathrm{H}), 7.44-7.43(\mathrm{~m}, 1 \mathrm{H}), 7.35-7.31(\mathrm{~m}, 3 \mathrm{H}), 7.15(\mathrm{~s}, 1 \mathrm{H})$, $6.44(\mathrm{~d}, \mathrm{~J}=3.2 \mathrm{~Hz}, 1 \mathrm{H}), 6.37-6.36(\mathrm{~m}, 1 \mathrm{H}), 5.29(\mathrm{dd}, \mathrm{J}=2.8,12.0 \mathrm{~Hz}, 1 \mathrm{H}), 4.24(\mathrm{~d}, \mathrm{~J}=10.8 \mathrm{~Hz}$, $1 \mathrm{H}), 4.12(\mathrm{~d}, \mathrm{~J}=11.2 \mathrm{~Hz}, 1 \mathrm{H}), 3.87(\mathrm{~s}, 1 \mathrm{H}), 3.34(\mathrm{~s}, 1 \mathrm{H}), 3.23(\mathrm{~s}, 2 \mathrm{H}), 2.40(\mathrm{~s}, 3 \mathrm{H}), 2.16(\mathrm{dd}, \mathrm{J}=$ $12.8,14.0 \mathrm{~Hz}, 1 \mathrm{H}), 1.70(\mathrm{dd}, \mathrm{J}=2.8,14.0 \mathrm{~Hz}, 1 \mathrm{H}) ;{ }^{13} \mathrm{C}-\mathrm{NMR}\left(100 \mathrm{MHz}, \mathrm{CDCl}_{3}\right): \delta=153.0$, $142.7,135.9,131.8,130.0,128.3,128.1,127.7,110.2,108.2,70.6,68.8,68.5,67.7,60.4,43.2$, 42.2, 28.5, 21, 14.1 IR (thin film): $v_{\max }=3379,2954,2887,1601,1495,1455,1311,1240,1151$, $1064,1033,914,866,814,736,704$; HRMS calc'd for $\mathrm{C}_{17} \mathrm{H}_{21} \mathrm{O}_{4} \mathrm{~N}=303.1471$, found 330.1474<smiles>CN1OC(c2ccco2)CC2(COC(C)(C)OC2)C1c1ccccc1</smiles>

\section{Compound $7 \mathrm{fc}$}

Compound $\mathbf{7 f \mathbf { c }}$ was prepared from compound $\mathbf{7} \mathbf{f b}$ utilizing the general procedure for Step C. Amounts of reagents included $580 \mathrm{mg}(1.91 \mathrm{mmol})$ of $7 \mathrm{fb}, 349 \mathrm{mg}(0.753 \mathrm{mmol})$ of 2methoxypropene, and $61 \mathrm{mg}(0.321 \mathrm{mmol})$ of p-toluenesulfonic acid monohydrate in $20 \mathrm{~mL}$ of DMF. Compound $7 \mathrm{fc}(611 \mathrm{mg}, 1.53 \mathrm{mmol}, 95 \%)$ was obtained as a foam oil. $\mathrm{R}_{\mathrm{f}}=0.6,30 \%$ 
ethyl acetate in hexanes; ${ }^{1} \mathrm{H}-\mathrm{NMR}\left(400 \mathrm{MHz}, \mathrm{CDCl}_{3}\right): \delta=8.02(\mathrm{~s}, 1 \mathrm{H}), 7.43(\mathrm{dd}, \mathrm{J}=0.8,6.0$ $\mathrm{Hz}, 1 \mathrm{H}), 7.33-7.28(\mathrm{~m}, 3 \mathrm{H}), 7.21(\mathrm{~s}, 1 \mathrm{H}), 6.39(\mathrm{~d}, \mathrm{~J}=3.6 \mathrm{~Hz}, 1 \mathrm{H}), 6.36(\mathrm{dd}, \mathrm{J}=1.8,3.2 \mathrm{~Hz}, 1 \mathrm{H})$, $5.08(\mathrm{dd}, \mathrm{J}=3.2,12.8 \mathrm{~Hz}, 1 \mathrm{H}), 4.47(\mathrm{~d}, \mathrm{~J}=11.2 \mathrm{~Hz}, 1 \mathrm{H}), 4.00(\mathrm{~s}, 1 \mathrm{H}), 3.97(\mathrm{~d}, 11.2 \mathrm{~Hz}, 1 \mathrm{H})$, $3.24(\mathrm{~d}, \mathrm{~J}=12.4 \mathrm{~Hz}, 1 \mathrm{H}), 3.17$ (dd, J = 1.4, $12.0 \mathrm{~Hz}, 1 \mathrm{H}), 2.45$ (s, 3H), 2.10 (dd, J = 12.4, 13.6 $\mathrm{Hz}, 1 \mathrm{H}), 1.65(\mathrm{~d}, \mathrm{~J}=14.0 \mathrm{~Hz}, 1 \mathrm{H}), 1.45(\mathrm{~s}, 3 \mathrm{H}), 1.41(\mathrm{~s}, 3 \mathrm{H}) ;{ }^{13} \mathrm{C}-\mathrm{NMR}\left(100 \mathrm{MHz}, \mathrm{CDCl}_{3}\right): \delta=$ $152.9,142.6,135.9,132.1,129.5,127.9,127.4,110.1,107.9,98.0,69.8,68.3,66.9,66.6,43.3$, 36.0, 29.3, 25.9, 21.3; IR (thin film): $v_{\max }=2990,2937,2863,1678,1495,1453,1373,1316$, $1260,1228,1200,1152,1087,1008,933,871,832,740$; HRMS calc'd for $\mathrm{C}_{20} \mathrm{H}_{25} \mathrm{O}_{4} \mathrm{~N}=$ 343.1784 , found 343.1780

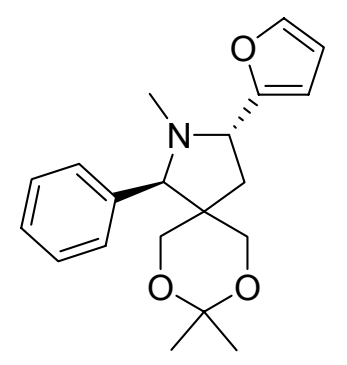

\section{Compound 7f}

Compound 7f was prepared from compound $7 \mathbf{f c}$ utilizing the general procedure for Steps D and E. Amounts of reagents Step D: $100 \mathrm{mg}(0.292 \mathrm{mmol})$ of Compound $7 \mathbf{f c}, 169 \mathrm{mg}(1.12 \mathrm{mmol})$ of Samarium metal, $259 \mathrm{mg}(1.02 \mathrm{mmol})$ of Iodine. Step E: $13 \mathrm{mg}(0.438 \mathrm{mmol})$ of $\mathrm{NaH}(80 \%$ dispersion in mineral oil), and $43 \mathrm{mg}(0.380 \mathrm{mmol})$ of methanesulfonyl chloride. Compound $7 \mathbf{f}$ (28mg, $0.084 \mathrm{mmol}, 29 \%$ for 2 steps) was obtained from $7 \mathbf{f c}$ as pale, pink crystals. MP $=109$ $110^{\circ} \mathrm{C} ; \mathrm{R}_{\mathrm{f}}=0.4,30 \%$ ethyl acetate in hexanes. ${ }^{1} \mathrm{H}-\mathrm{NMR}\left(400 \mathrm{MHz}, \mathrm{CDCl}_{3}\right): \delta=7.39(\mathrm{~s}, 1 \mathrm{H})$, 7.37-7.31 (m, 2H), 7.28-7.24 (m, 3H), $6.29(\mathrm{dd}, \mathrm{J}=1.6,3.2 \mathrm{~Hz}, 1 \mathrm{H}), 6.18(\mathrm{~d}, \mathrm{~J}=2.8 \mathrm{~Hz}, 1 \mathrm{H})$, $4.41(\mathrm{dd}, \mathrm{J}=3.2,9.2 \mathrm{~Hz}, 1 \mathrm{H}), 4.00(\mathrm{~d}, \mathrm{~J}=11.2 \mathrm{~Hz}, 1 \mathrm{H}), 3.93(\mathrm{dd}, \mathrm{J}=2.0,11.2 \mathrm{~Hz}, 1 \mathrm{H}), 3.67(\mathrm{~s}$, $1 \mathrm{H}), 3.40(\mathrm{dd}, \mathrm{J}=2.0,11.6 \mathrm{~Hz}, 1 \mathrm{H}), 3.18(\mathrm{~d}, \mathrm{~J}=11.6 \mathrm{~Hz}, 1 \mathrm{H}), 2.48(\mathrm{dd}, \mathrm{J}=9.2,14.0 \mathrm{~Hz}, 1 \mathrm{H})$, $2.23(\mathrm{dd}, \mathrm{J}=2.8,14.0 \mathrm{~Hz}, 1 \mathrm{H}), 1.96(\mathrm{~s}, 3 \mathrm{H}), 1.32(\mathrm{~s}, 3 \mathrm{H}), 1.32(\mathrm{~s}, 3 \mathrm{H}), 1.18(\mathrm{~s}, 3 \mathrm{H}) ;{ }^{13} \mathrm{C}-\mathrm{NMR}$ $\left(100 \mathrm{MHz}, \mathrm{CDCl}_{3}\right): \delta=156.7,141.9,138.7,128.2,127.3,109.8,107.8,97.7,72.9,67.7,67.5$, $59.3,43.6,38.2,35.6,26.9,20.5$; *Note- 1 aromatic peak appears to be absent, presumably due to overlap: IR (thin film): $v_{\max }=2990,2962,2885,2867,290,1493,1369,1258,1197,1162$, 1145, 1103, 1069, 1027, 1016, 932, 744, 704: HRMS calc'd for $\mathrm{C}_{20} \mathrm{H}_{25} \mathrm{O}_{3} \mathrm{~N}=327.1834$, found 327.1833; Relative stereochemistry confirmed by X-ray crystallography.<smiles>COC(=O)C1(C(C)=O)C[C@H](C)ON(C)[C@H]1c1ccccc1</smiles>

Compound 7ga 
Compound 7ga was prepared from methyl nitrone and methyl cyclopropane diester utilizing the general procedure for Step A. Amounts of reagents included $736 \mathrm{mg}(5.44 \mathrm{mmol})$ of methyl nitrone, $670 \mathrm{mg}(3.90 \mathrm{mmol})$ methyl cyclopropane diester, and $482 \mathrm{mg}(0.777 \mathrm{mmol})$ of ytterbium triflate and $40 \mathrm{~mL}$ of $\mathrm{CH}_{2} \mathrm{Cl}_{2}$. Compound $7 \mathrm{ga}(896 \mathrm{mg}, 2.92 \mathrm{mmol}$, 76\%) was obtained as colorless crystals. $\mathrm{MP}=82-84{ }^{\circ} \mathrm{C} ; \mathrm{R}_{\mathrm{f}}=0.5,30 \%$ ethyl acetate in hexanes. ${ }^{1} \mathrm{H}-$ NMR (400 MHz, $\left.\mathrm{CDCl}_{3}\right): \delta=7.50(\mathrm{~s}, 2 \mathrm{H}), 7.28-7.26(\mathrm{~m}, 3 \mathrm{H}), 4.69(\mathrm{~s}, 1 \mathrm{H}), 3.97-3.90(\mathrm{~m}, 1 \mathrm{H})$, $3.80(\mathrm{~s}, 3 \mathrm{H}), 3.35(\mathrm{~s}, 3 \mathrm{H}), 2.41(\mathrm{~s}, 3 \mathrm{H}), 2.30-2.26(\mathrm{~m}, 2 \mathrm{H}), 1.30(\mathrm{~d}, \mathrm{~J}=6.0 \mathrm{~Hz}, 3 \mathrm{H})$; ${ }^{13} \mathrm{C}-\mathrm{NMR}$ $\left(100 \mathrm{MHz}, \mathrm{CDCl}_{3}\right): \delta=170.34,168.5,134.6,130.9,127.9,128.1,71.9,67.6,58.9,53.2,52.3$, 43.2, 31.7, 20.2; IR (thin film): $v_{\max }=2955,2885,1742,1493,1435,1381,1258,1201,1118$, 1041, 953, 919, 822, 772, 703; HRMS calc'd for $\mathrm{C}_{16} \mathrm{H}_{21} \mathrm{O}_{5} \mathrm{~N}=359.1369$, found 359.1373<smiles>C[C@H]1CC(C)(CO)[C@@H](c2ccccc2)N(C)O1</smiles>

\section{Compound $7 \mathrm{gb}$}

Compound 7gb was prepared from compound 7ga utilizing the general procedure for Step B. Amounts of reagents included $785 \mathrm{mg}(2.55 \mathrm{mmol})$ of $7 \mathrm{ga}$ and $5.1 \mathrm{~mL}(5.1 \mathrm{mmol})$ of $1 \mathrm{M}$ lithium aluminum hydride in $20 \mathrm{~mL}$ of THF. Compound 7gb (570 mg, $2.26 \mathrm{mmol}, 89 \%)$ was obtained as a colorless powder. $\mathrm{MP}=106-108{ }^{\circ} \mathrm{C} ; \mathrm{R}_{\mathrm{f}}=0.2,70 \%$ ethyl acetate in hexanes; ${ }^{1} \mathrm{H}-\mathrm{NMR}(400$ $\left.\mathrm{MHz} \mathrm{CDCl}_{3}\right): \delta=7.87(\mathrm{~d}, \mathrm{~J}=6.4 \mathrm{~Hz}, 1 \mathrm{H}), 7.32-7.24(\mathrm{~m}, 3 \mathrm{H}), 7.11(\mathrm{~s}, 1 \mathrm{H}), 4.25-4.19(\mathrm{~m}, 1 \mathrm{H})$, $4.14(\mathrm{~d}, \mathrm{~J}=10.8 \mathrm{~Hz}, 1 \mathrm{H}), 3.97$ (d, J = 10.8 Hz, 1H), 3.76 (s, 1H), 3.07 (s, 2H), 2.30 (s, 3H), 1.44$1.40(\mathrm{~m}, 2 \mathrm{H}), 1.22(\mathrm{~d}, \mathrm{~J}=6.4 \mathrm{~Hz}, 3 \mathrm{H}) ;{ }^{13} \mathrm{C}-\mathrm{NMR}\left(100 \mathrm{MHz}, \mathrm{CDCl}_{3}\right): \delta=136.1,131.9,129.8$, $128.0,127.6,71.1,68.6,68.1,67.6,43.1,42.1,32.9,20.7$; IR (thin film): $v_{\max }=3379,2930$, 2886, 1601, 1492, 1454, 1377, 1260, 1144, 1071, 1041, 967, 910, 864, 817, 759, 734, 703, 638; HRMS calc'd for $\mathrm{C}_{14} \mathrm{H}_{21} \mathrm{O}_{3} \mathrm{~N}=251.1521$, found 251.1523<smiles>C[C@@H]1CC2(COC(C)(C)OC2)[C@@H](c2ccccc2)N(C)O1</smiles>

\section{Compound 7gc}

Compound 7gc was prepared from compound 7gb utilizing the general procedure for Step C. Amounts of reagents included $540 \mathrm{mg}(2.15 \mathrm{mmol})$ of $7 \mathrm{gb}, 465 \mathrm{mg}(6.45 \mathrm{mmol})$ of 2methoxypropene, and $82 \mathrm{mg}(0.43 \mathrm{mmol})$ of p-toluenesulfonic acid monohydrate in $20 \mathrm{~mL}$ of DMF. Compound 7gc (577mg, $1.98 \mathrm{mmol}, 92 \%)$ was obtained as a colorless powder. MP = 96- 
$99{ }^{\circ} \mathrm{C} ; \mathrm{R}_{\mathrm{f}}=0.6,30 \%$ ethyl acetate in hexanes. ${ }^{1} \mathrm{H}-\mathrm{NMR}\left(400 \mathrm{MHz}, \mathrm{CDCl}_{3}\right): \delta=7.89(\mathrm{~s}, 1 \mathrm{H})$, 7.32-7.26 (m, 3H), 7.22 (s, 1H), $4.41(\mathrm{~d}, \mathrm{~J}=11.2 \mathrm{~Hz}, 1 \mathrm{H}), 4.13-4.08(\mathrm{~m}, 1 \mathrm{H}), 3.96(\mathrm{~s}, \mathrm{H}), 3.88$ (d, J = 11.2 Hz, 1H), $3.17(\mathrm{~d}, \mathrm{~J}=11.6 \mathrm{~Hz}, 1 \mathrm{H}), 3.07(\mathrm{dd}, \mathrm{J}=1.6,12.0 \mathrm{~Hz}, 1 \mathrm{H}), 2.40(\mathrm{~s}, 3 \mathrm{H}), 1.43$ $(\mathrm{s}, 3 \mathrm{H}), 1.41-1.38(\mathrm{~m}, 2 \mathrm{H}), 1.23(\mathrm{~d}, \mathrm{~J}=6.4 \mathrm{~Hz}, 3 \mathrm{H}) ;{ }^{13} \mathrm{C}-\mathrm{NMR}\left(100 \mathrm{MHz}, \mathrm{CDCl}_{3}\right): \delta=136.4$, 132.2, 129.5, 127.9, 127.4, 98.0, 70.4, 68.2, 67.1, 67.0, 43.3, 36.2, 33.7, 26.3, 21.3, 20.6;. IR (thin film): $v_{\max }=2988,2970,2924,2881,1452,1372,1260,1202,1150,665$; HRMS calc'd for $\mathrm{C}_{17} \mathrm{H}_{25} \mathrm{O}_{3} \mathrm{~N}=291.1834$, found 291.1834

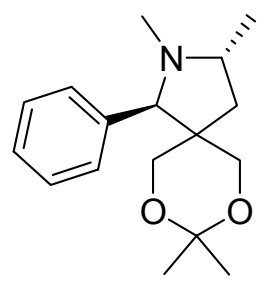

\section{Compound $7 \mathrm{~g}$}

Compound $7 \mathbf{g}$ was prepared from compound $7 \mathbf{g c}$ utilizing the general procedure for Steps D and E. Amounts of reagents Step D: $200 \mathrm{mg}(0.687 \mathrm{mmol})$ of Compound $7 \mathbf{g c}, 398 \mathrm{mg}(2.65 \mathrm{mmol})$ of Samarium metal, $610 \mathrm{mg}(2.40 \mathrm{mmol})$ of Iodine. Step E: $31 \mathrm{mg}(1.03 \mathrm{mmol})$ of $\mathrm{NaH}(80 \%$ dispersion in mineral oil), and $102 \mathrm{mg}(0.893 \mathrm{mmol})$ of methanesulfonyl chloride. Compound $7 \mathbf{g}$ (121 mg, $0.440 \mathrm{mmol}, 64 \%$ two steps) was obtained from $7 \mathbf{g c}$ as colorless needles. MP $=103$ $105^{\circ} \mathrm{C} ; \mathrm{R}_{\mathrm{f}}=0.3,30 \%$ ethyl acetate in hexanes. ${ }^{1} \mathrm{H}-\mathrm{NMR}\left(400 \mathrm{MHz}, \mathrm{CDCl}_{3}\right): \delta=7.31-7.27(\mathrm{~m}$, 2H), 7.24-7.22 (m, 1H), 7.20-7.17 (m, 2H), $3.93(\mathrm{~d}, \mathrm{~J}=11.2 \mathrm{~Hz}, 1 \mathrm{H}), 3.62(\mathrm{dd}, \mathrm{J}=2.0,11.2 \mathrm{~Hz}$, $1 \mathrm{H}), 3.54(\mathrm{~s}, 1 \mathrm{H}), 3.45-3.41(\mathrm{~m}, 1 \mathrm{H}), 3.10(\mathrm{~d}, \mathrm{~J}=11.6 \mathrm{~Hz}, 1 \mathrm{H}), 2.20(\mathrm{dd}, \mathrm{J}=8.4,13.6 \mathrm{~Hz}, 1 \mathrm{H})$, $2.10(\mathrm{~s}, 3 \mathrm{H}), 1.67(\mathrm{dd}, \mathrm{J}=3.2,13.6 \mathrm{~Hz}, 1 \mathrm{H}), 1.30(\mathrm{~s}, 3 \mathrm{H}), 1.17(\mathrm{~s}, 3 \mathrm{H}), 1.05(\mathrm{~d}, \mathrm{~J}=6.4 \mathrm{~Hz}, 3 \mathrm{H})$; ${ }^{13} \mathrm{C}-\mathrm{NMR}\left(100 \mathrm{MHz}, \mathrm{CDCl}_{3}\right): \delta=138.9,128.1,127.2$, 97.6, 72.7,69.3, 67.3, 57.0, 43.6, 40.9, $35.1,25.9,21.5,17.2$; *Note-One carbon signal is missing from the aromatic region presumably due to broad signals and overlap. IR (thin film): $v_{\max }=2964,2940,2860,1371,1259,1197$, 1158, 1111, 1076, 1033, 1014, 833, 726, 702, 648; HRMS calc'd for $\mathrm{C}_{17} \mathrm{H}_{25} \mathrm{O}_{2} \mathrm{~N}=275.1885$, found 275.18 


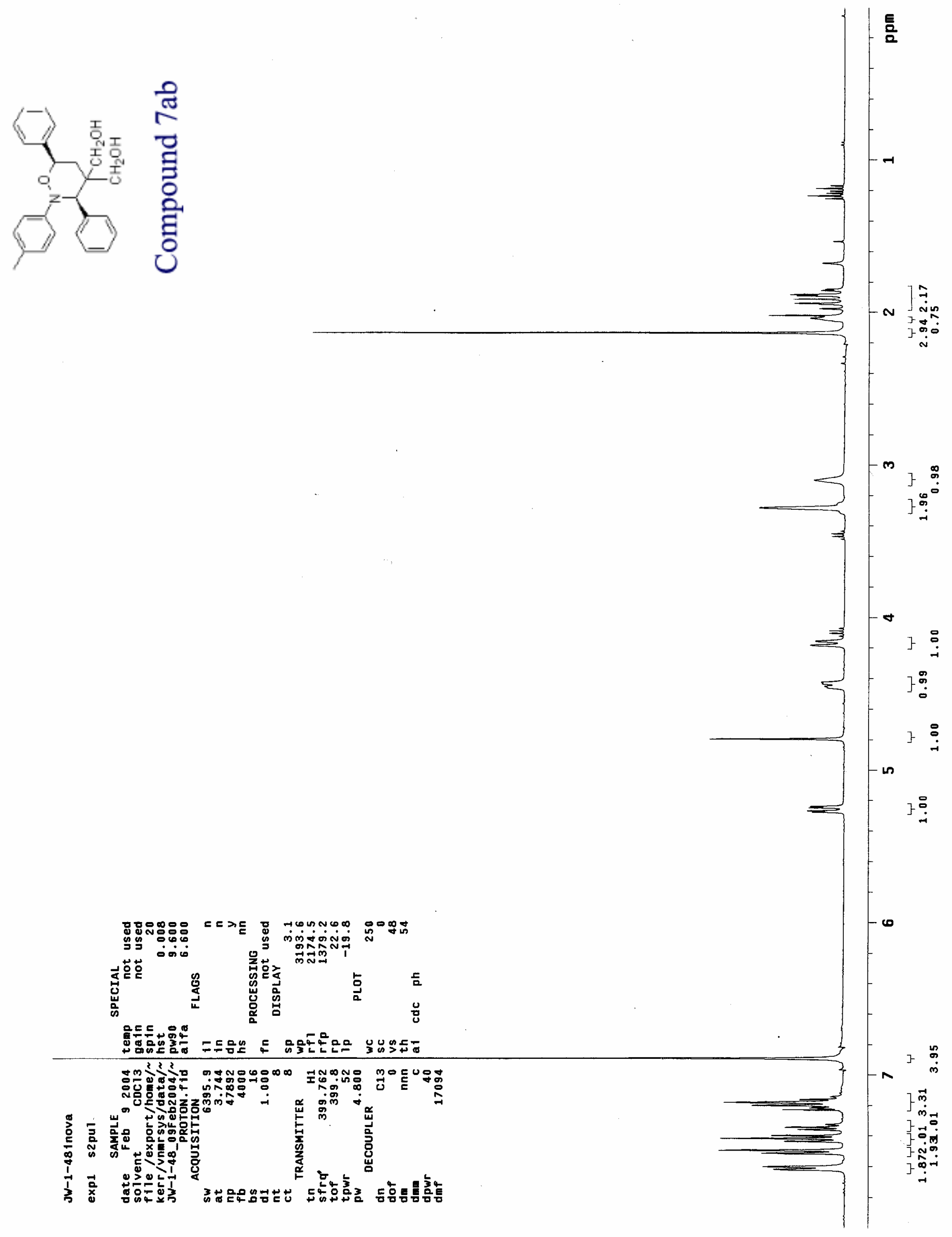



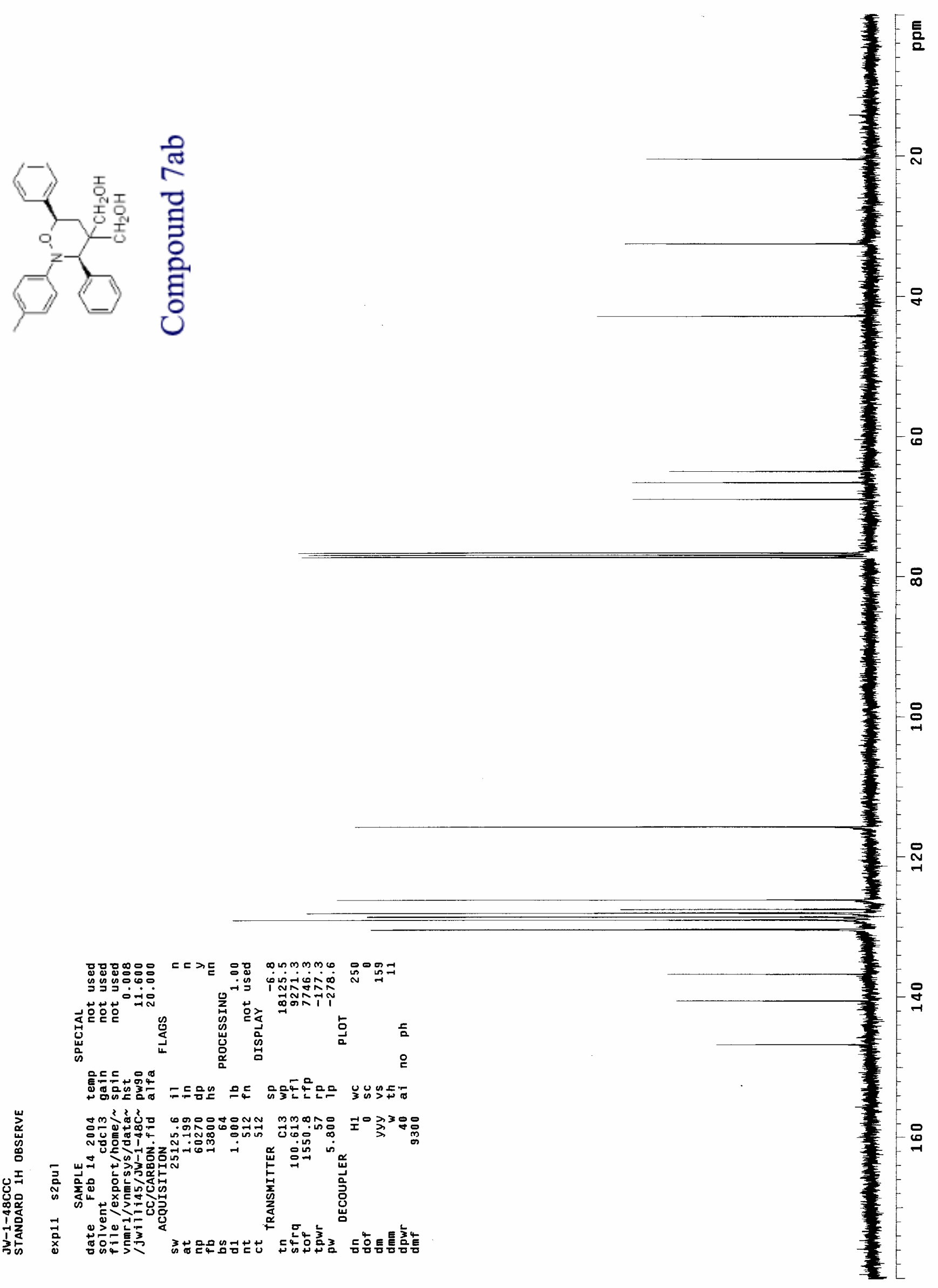

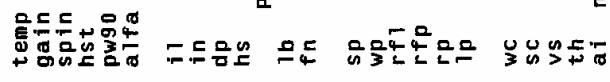

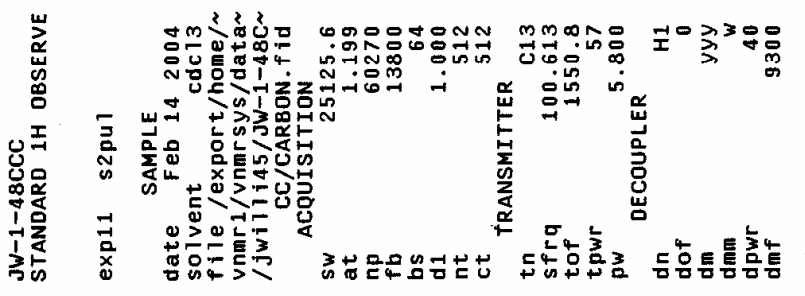




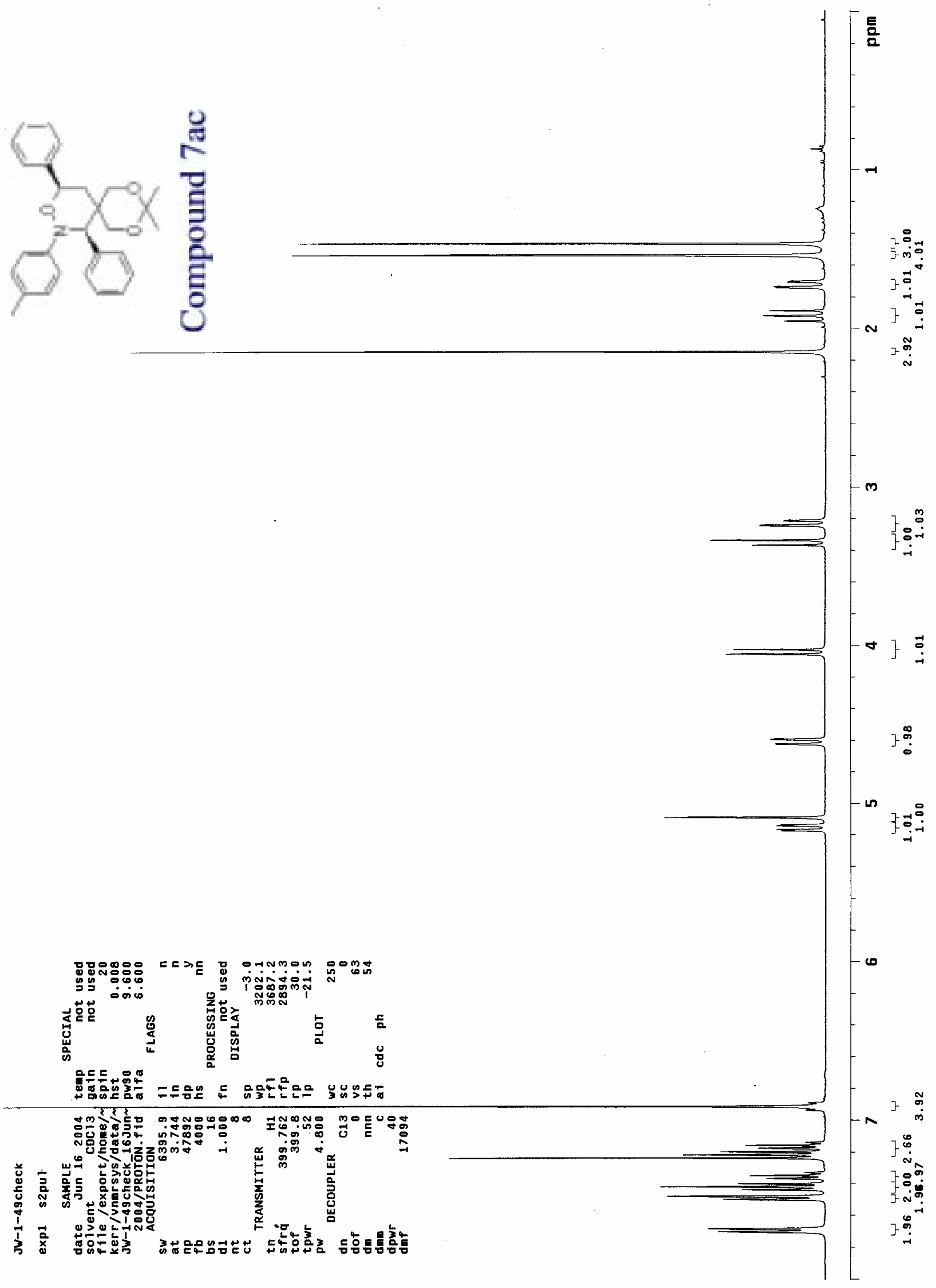



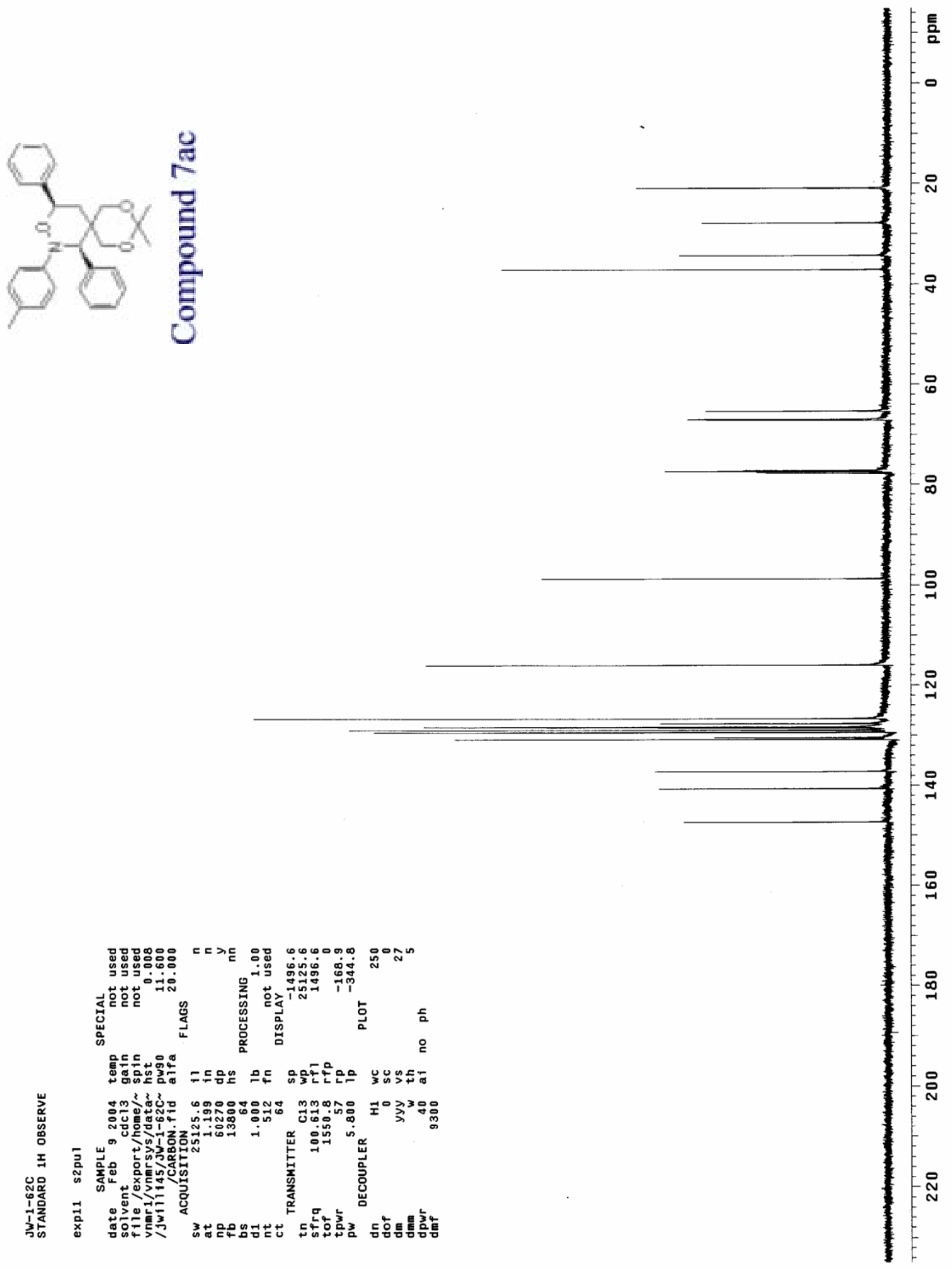


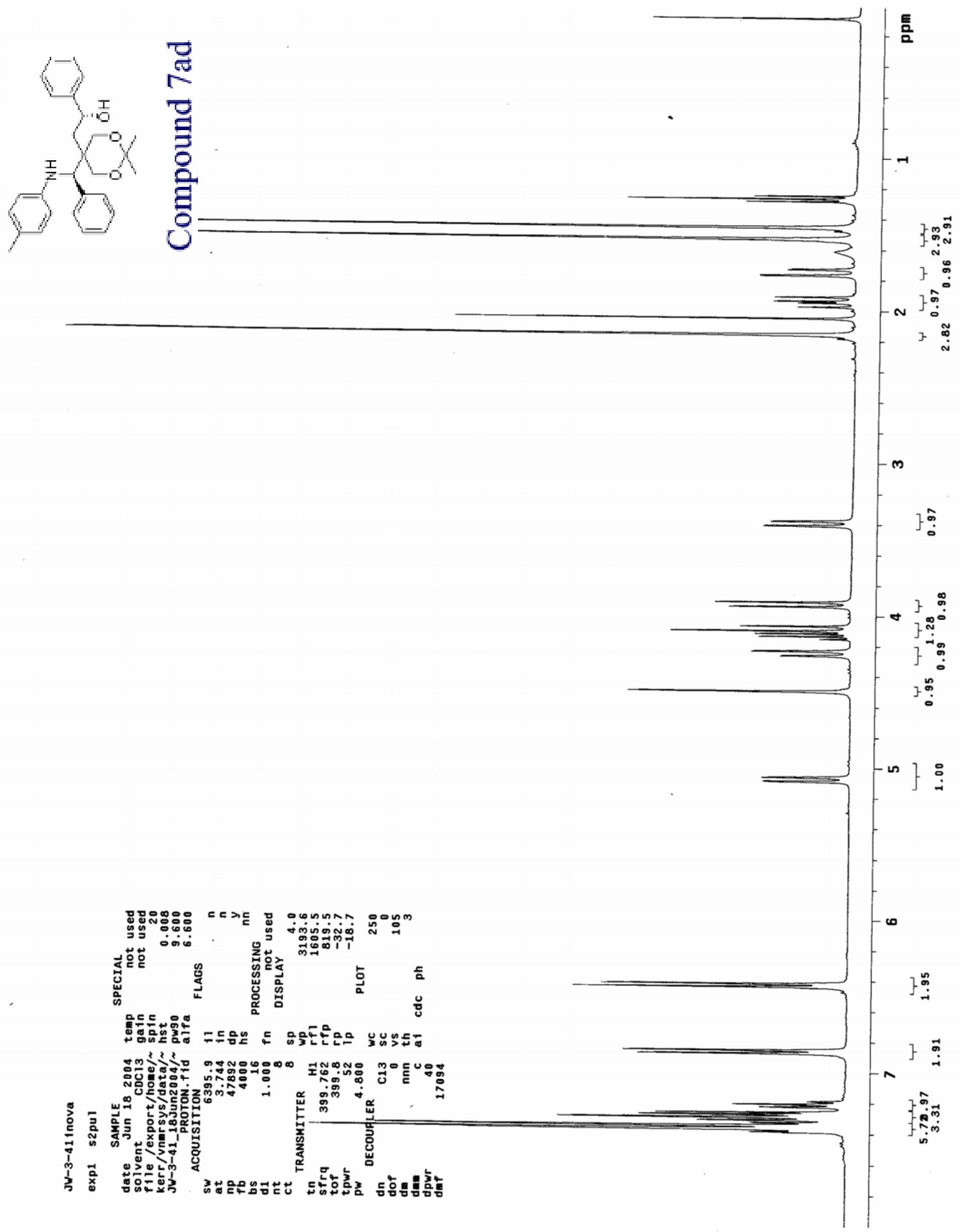



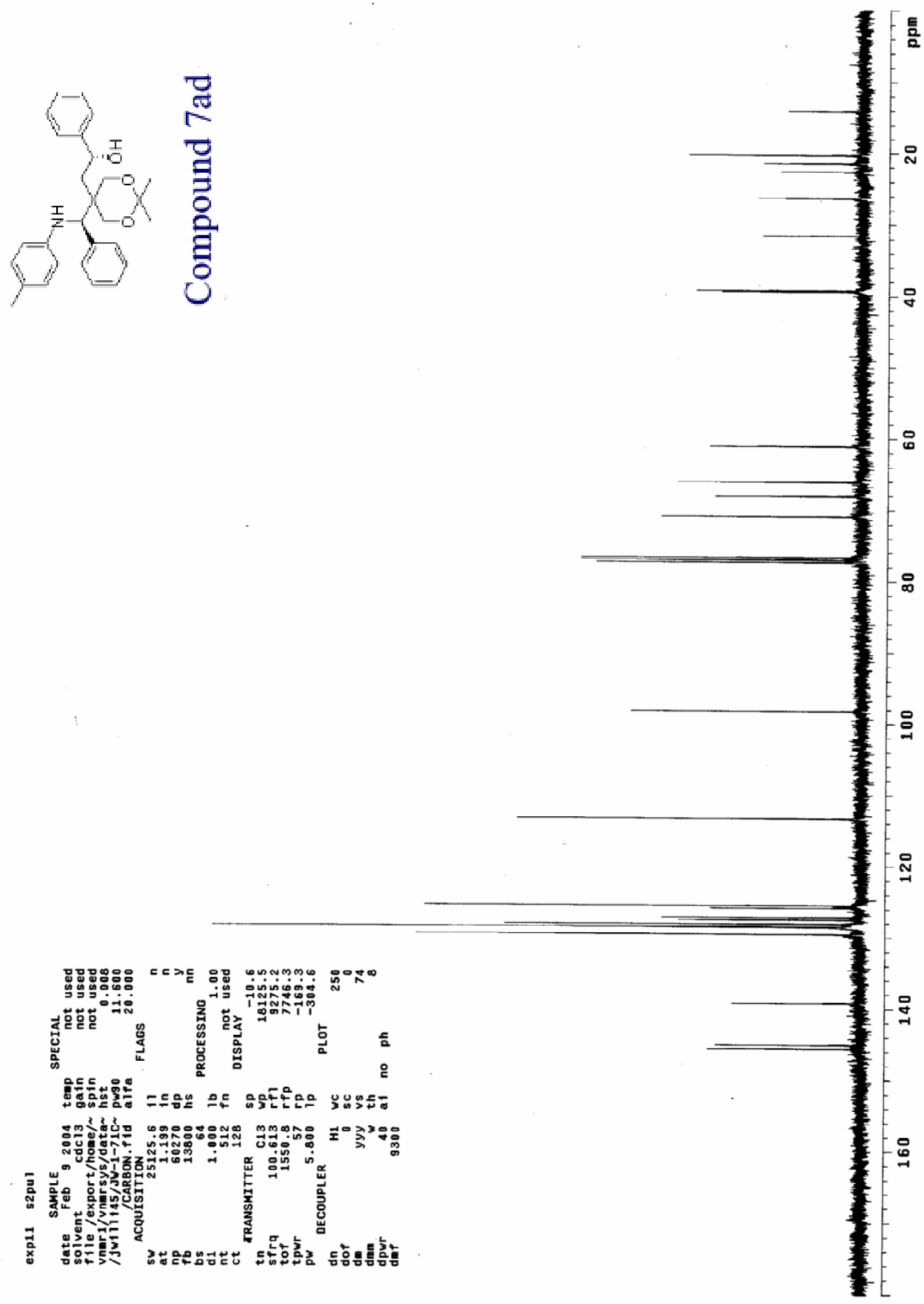

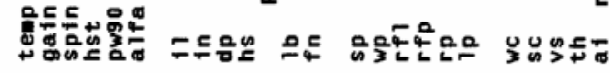

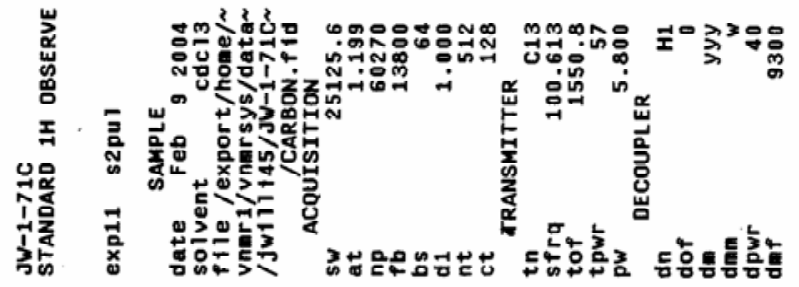




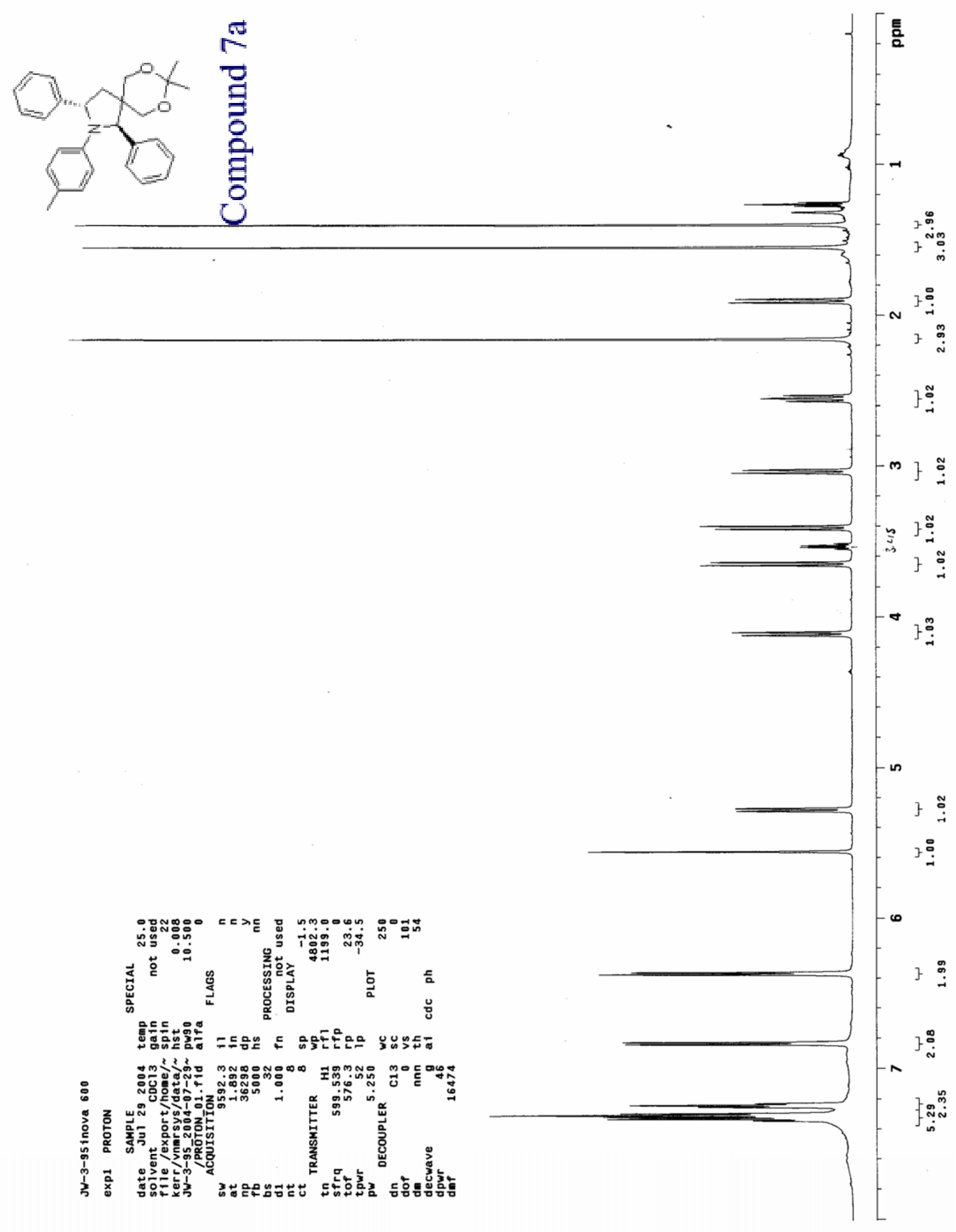



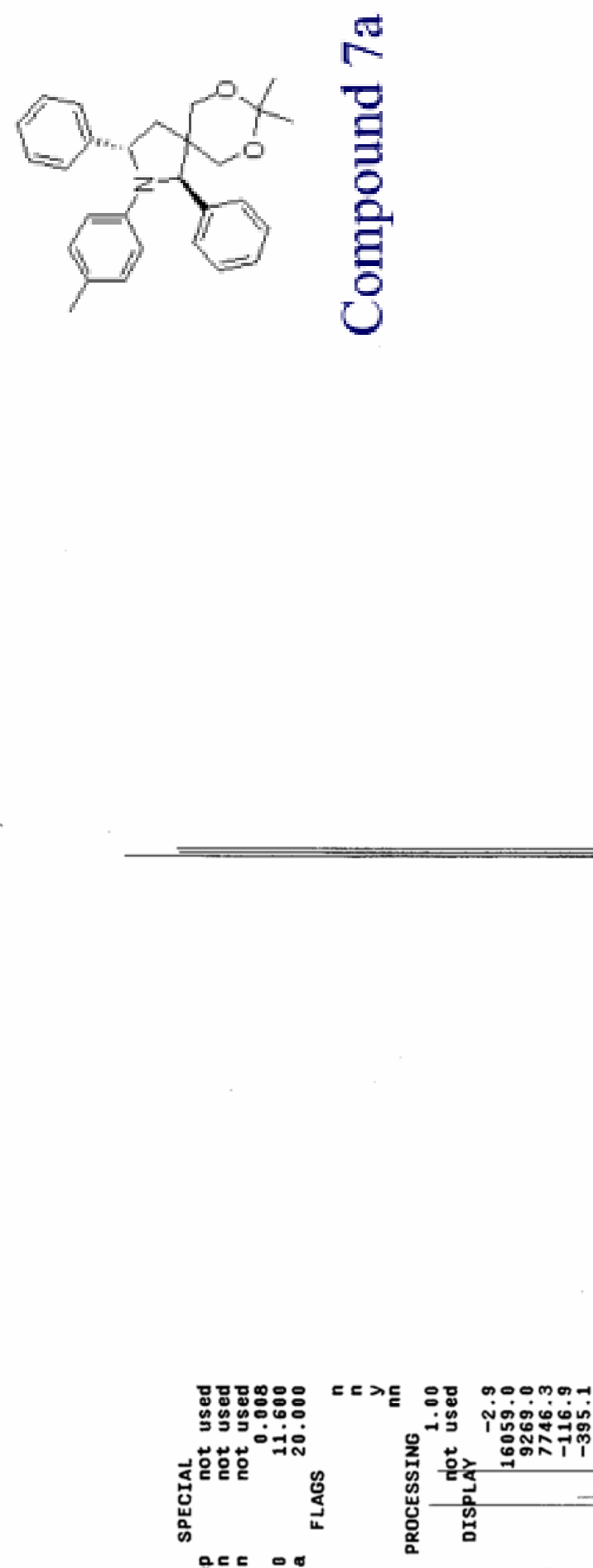

\section{串㹏}

$\frac{5}{2}$

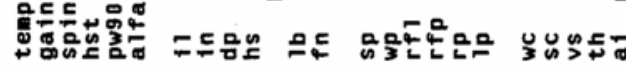

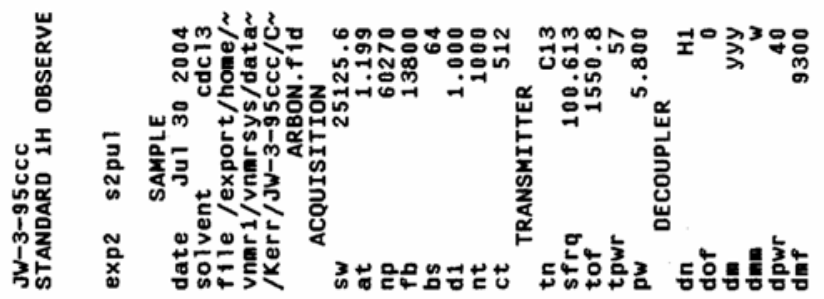




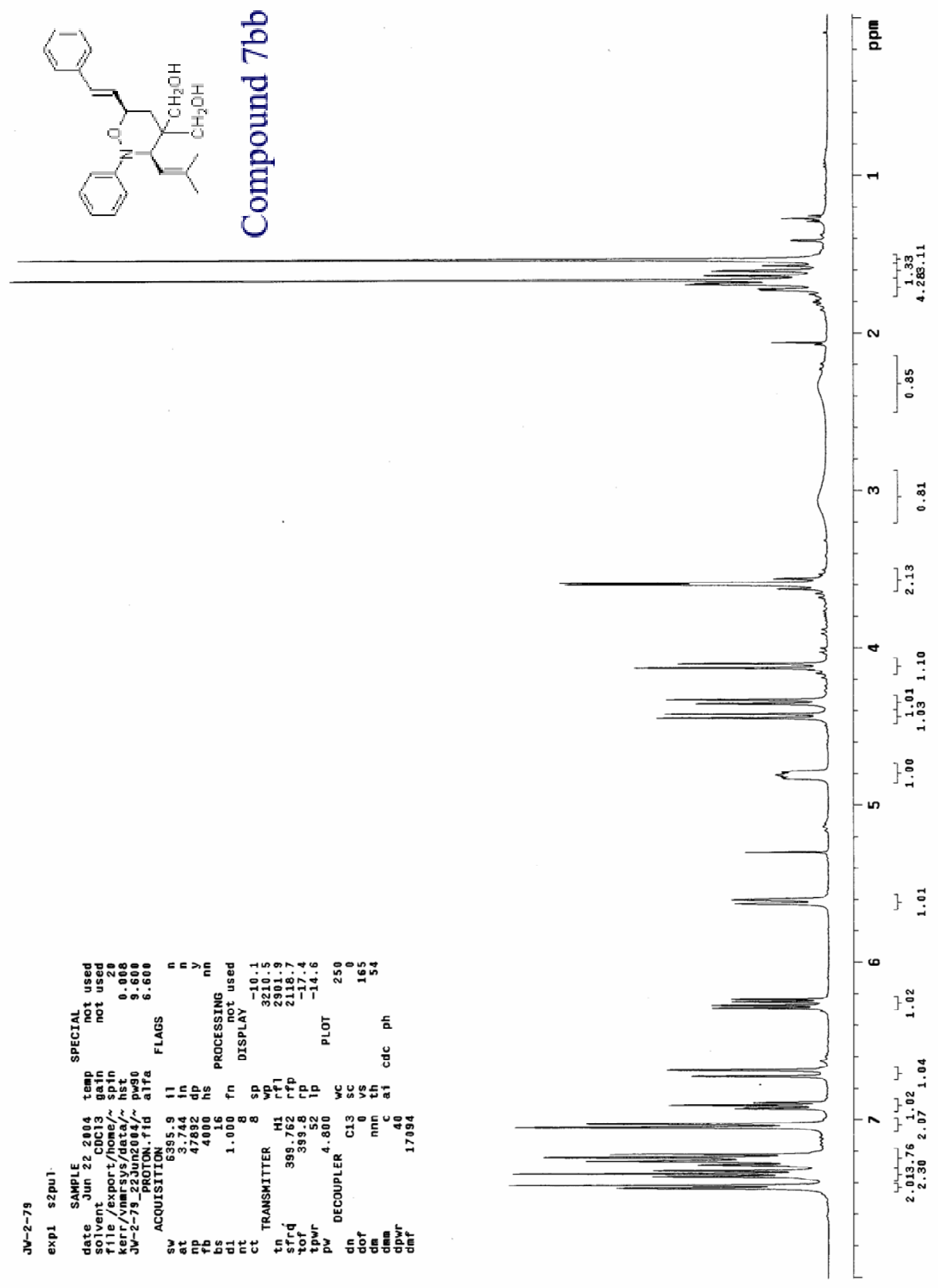



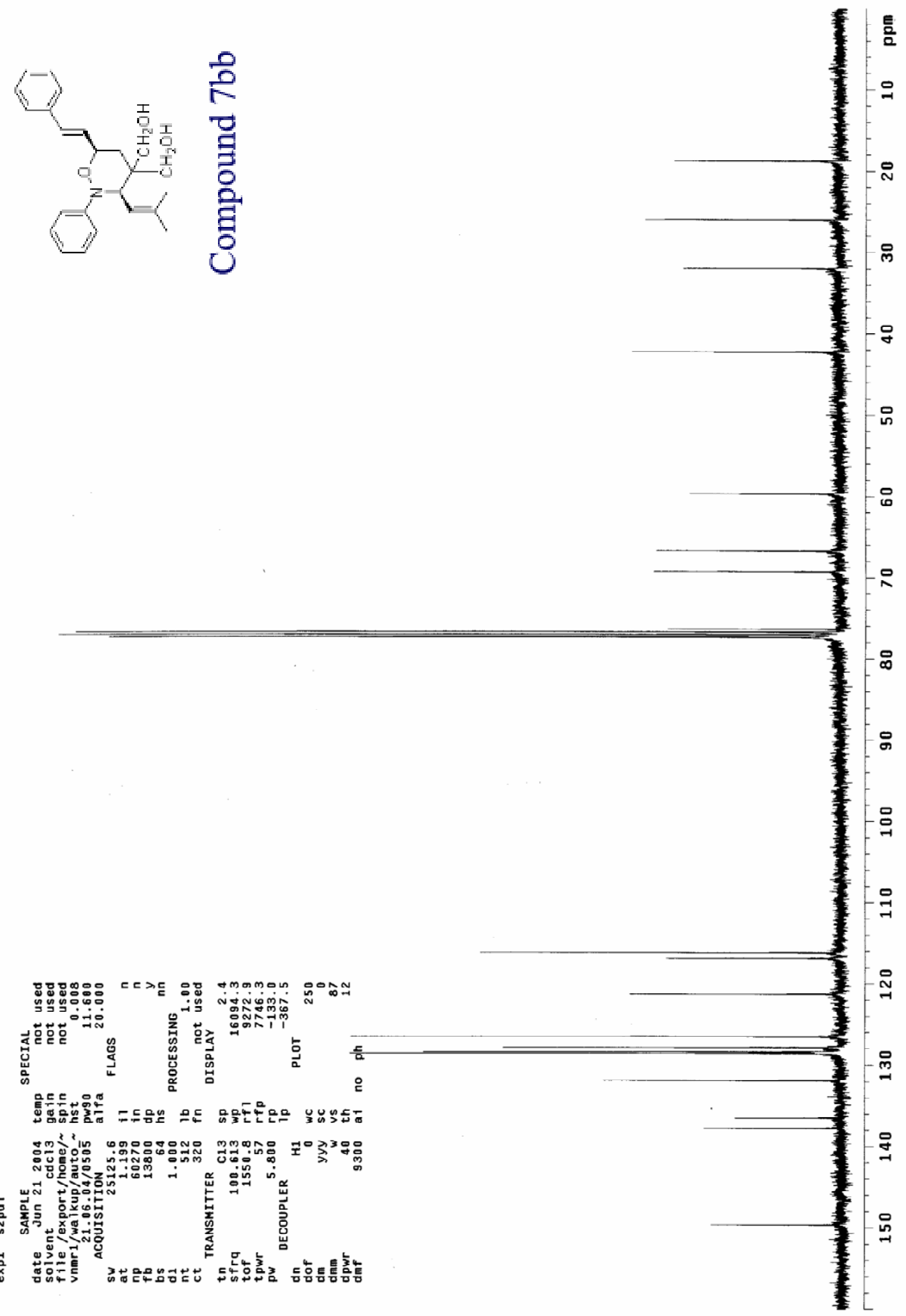


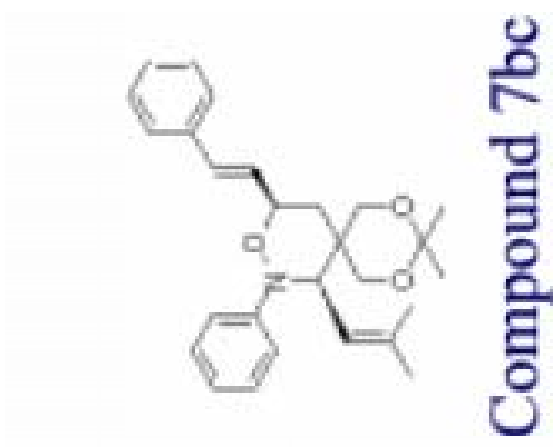

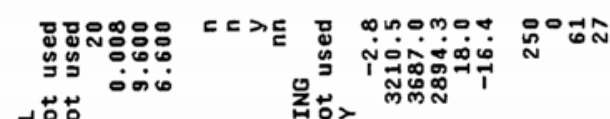

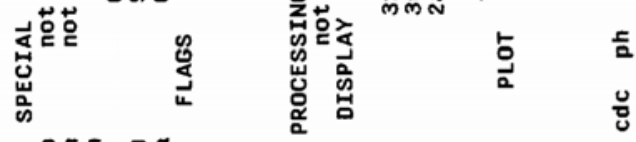

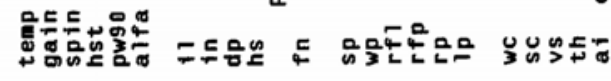
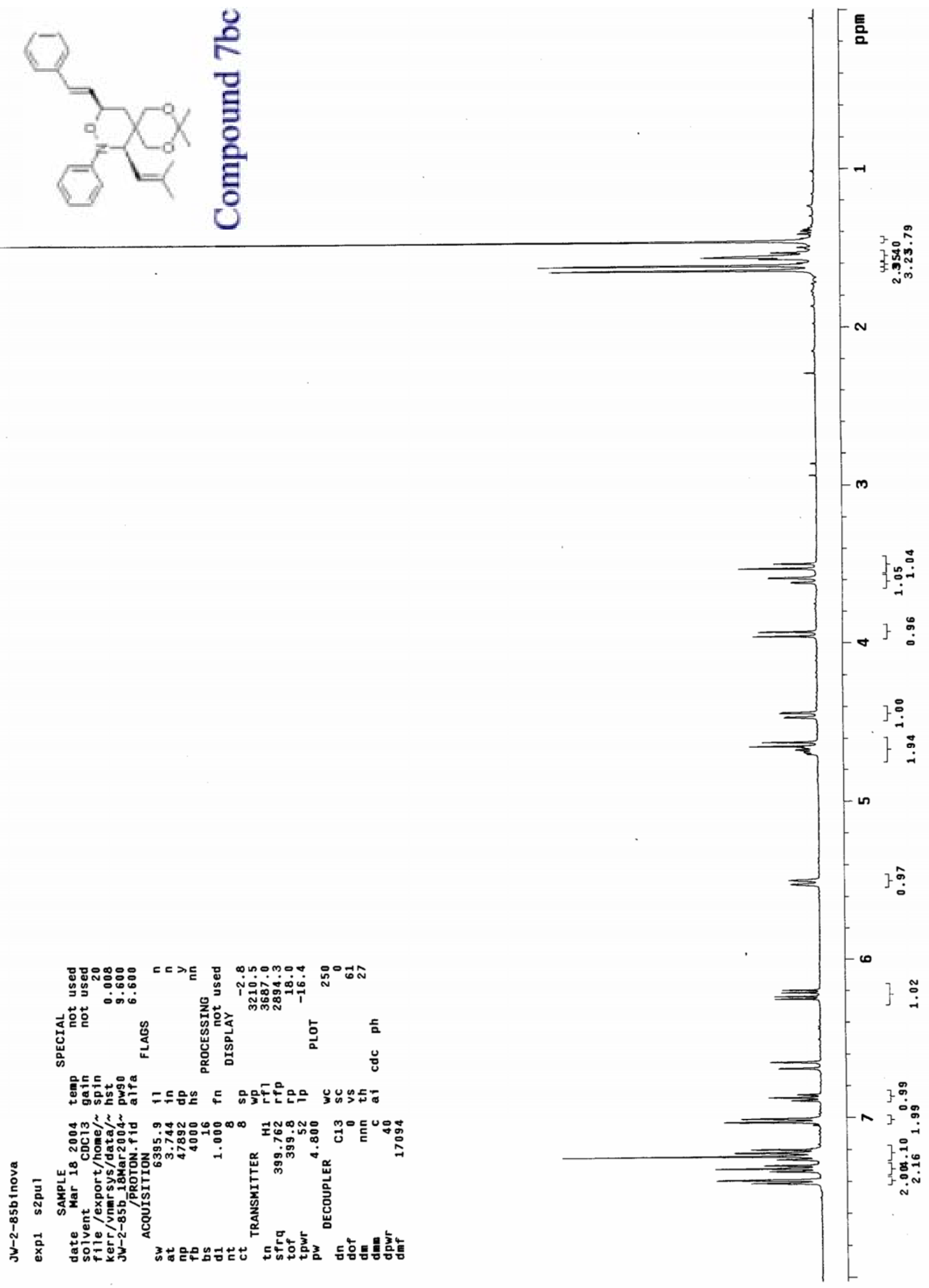

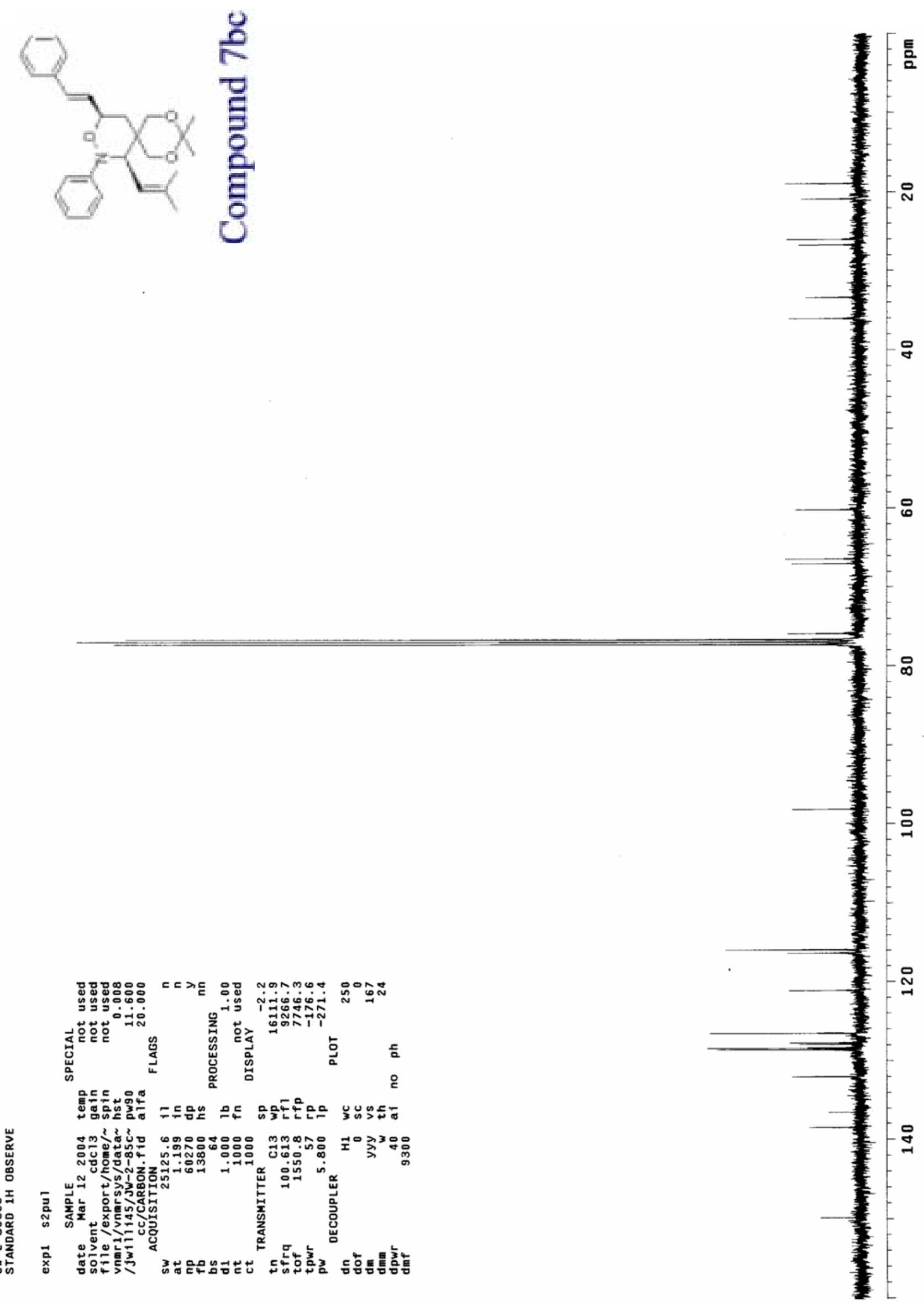

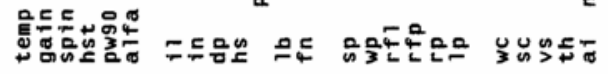

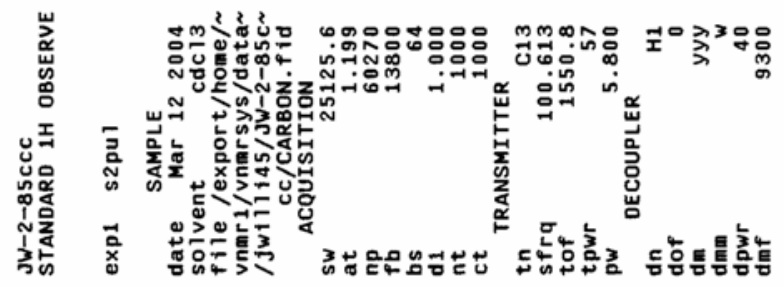




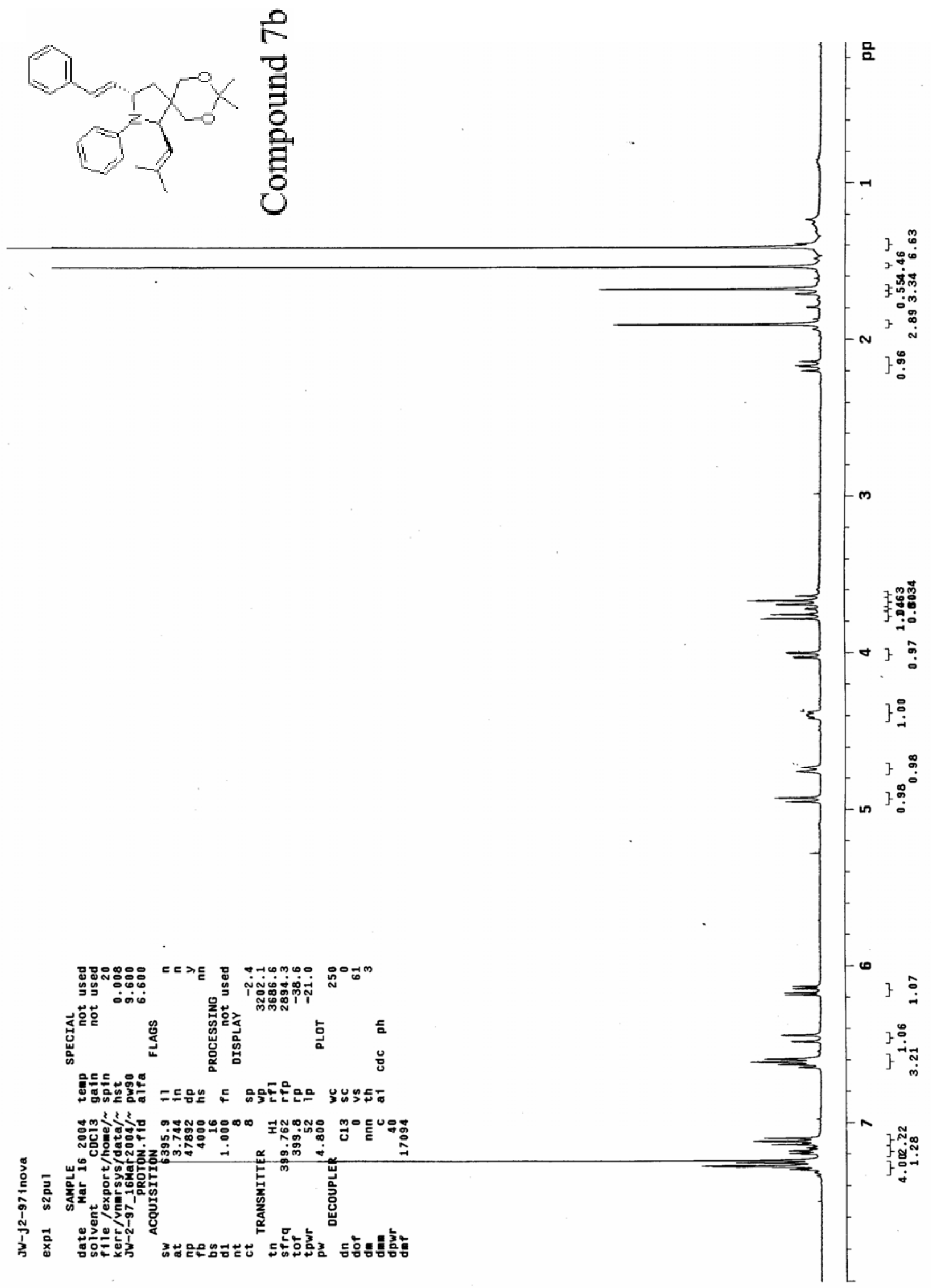




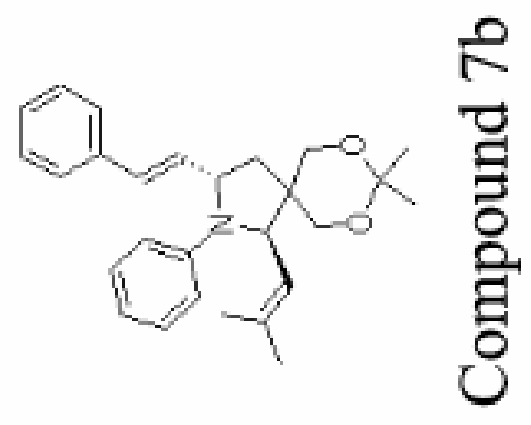

를
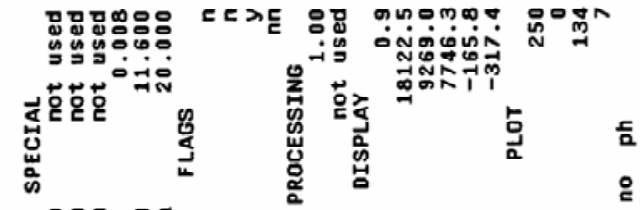

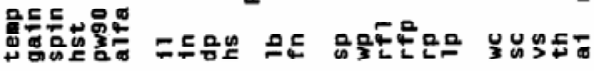

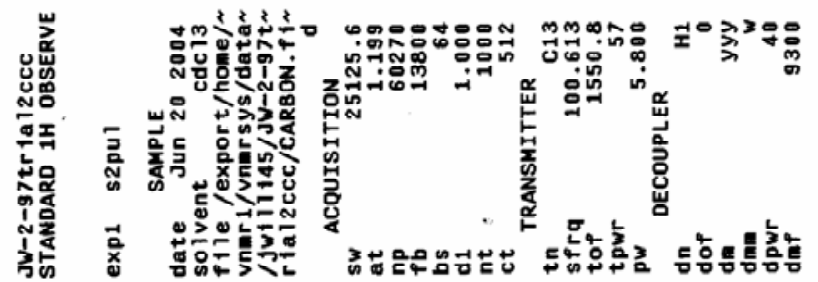




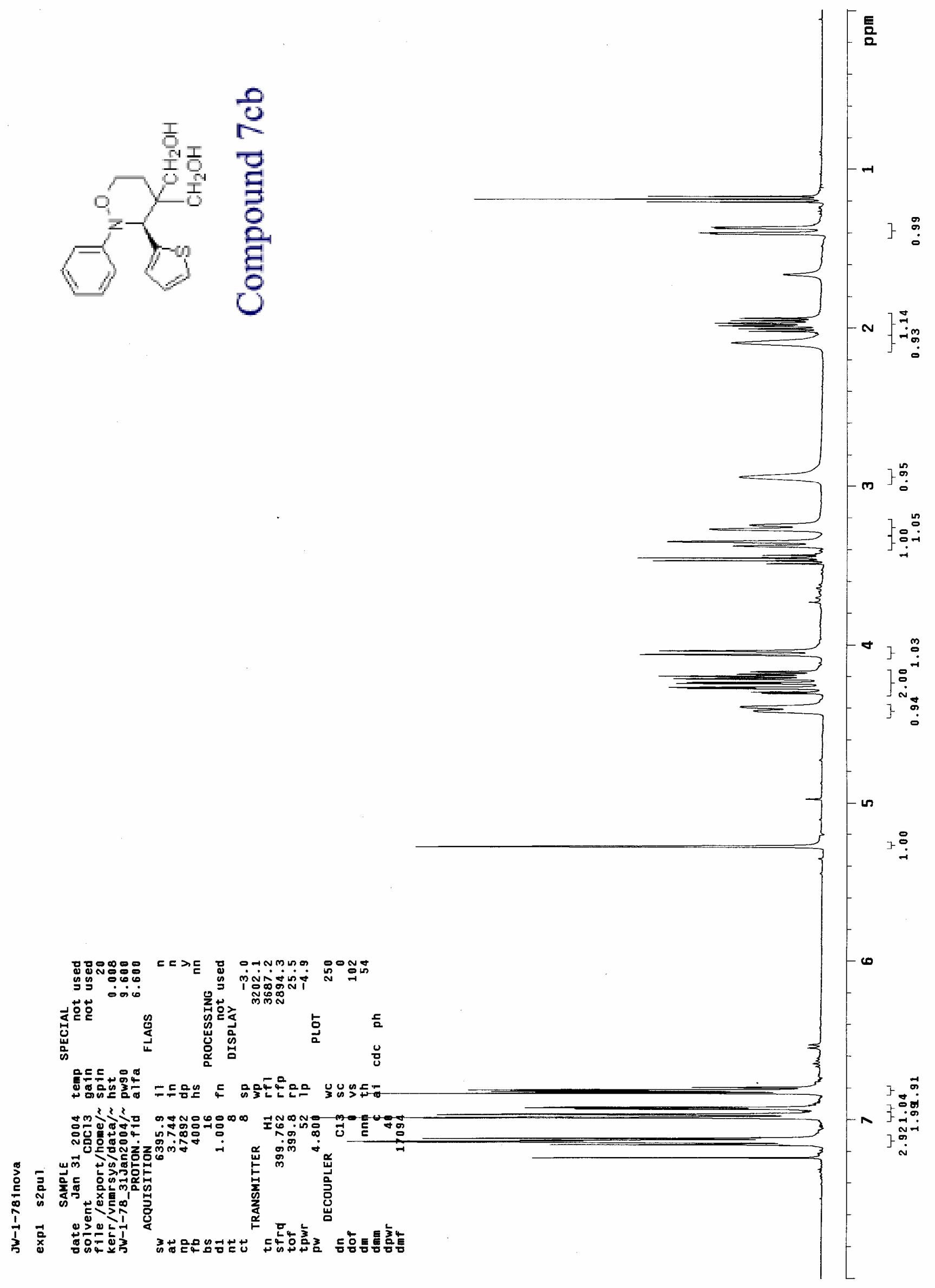



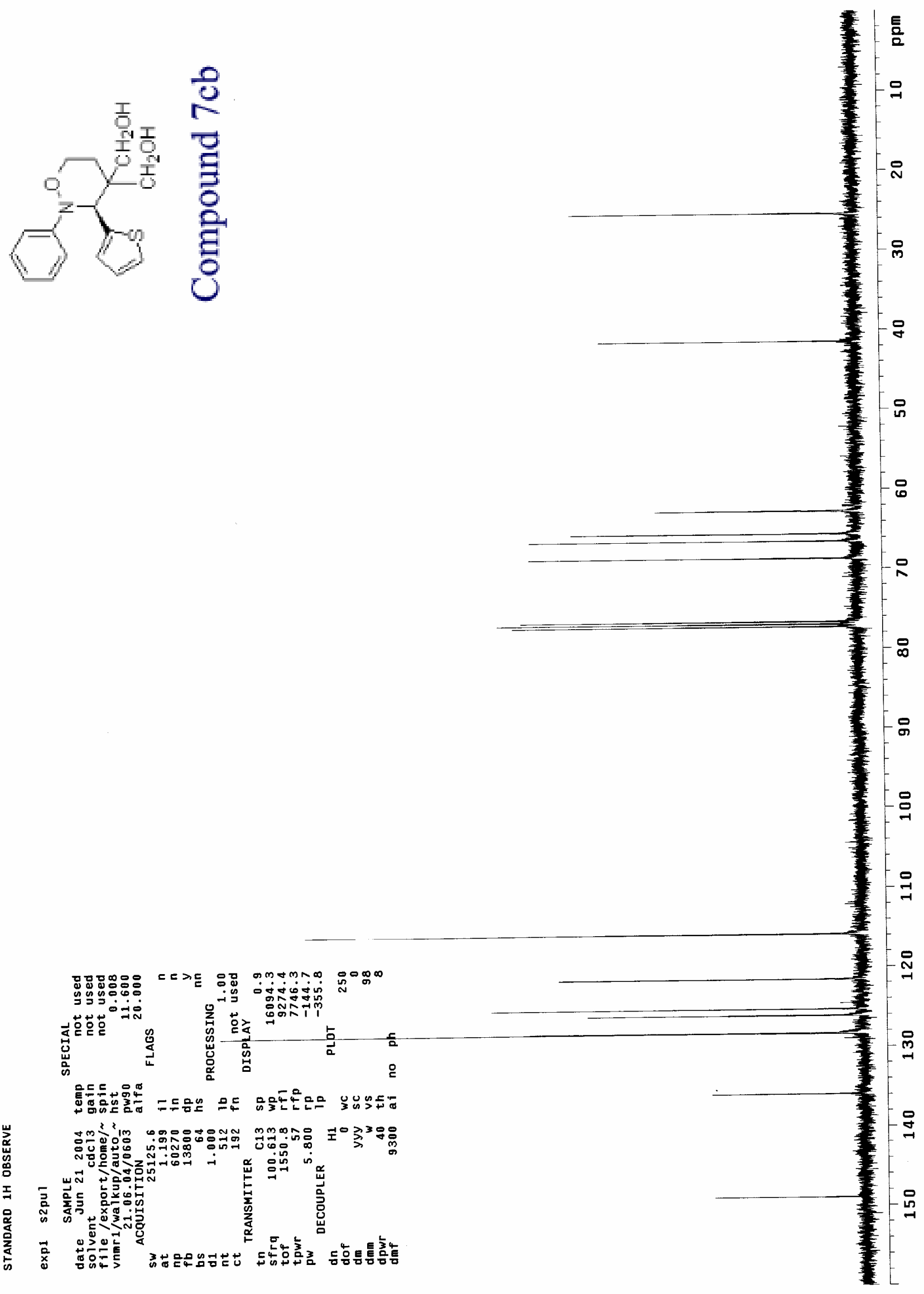

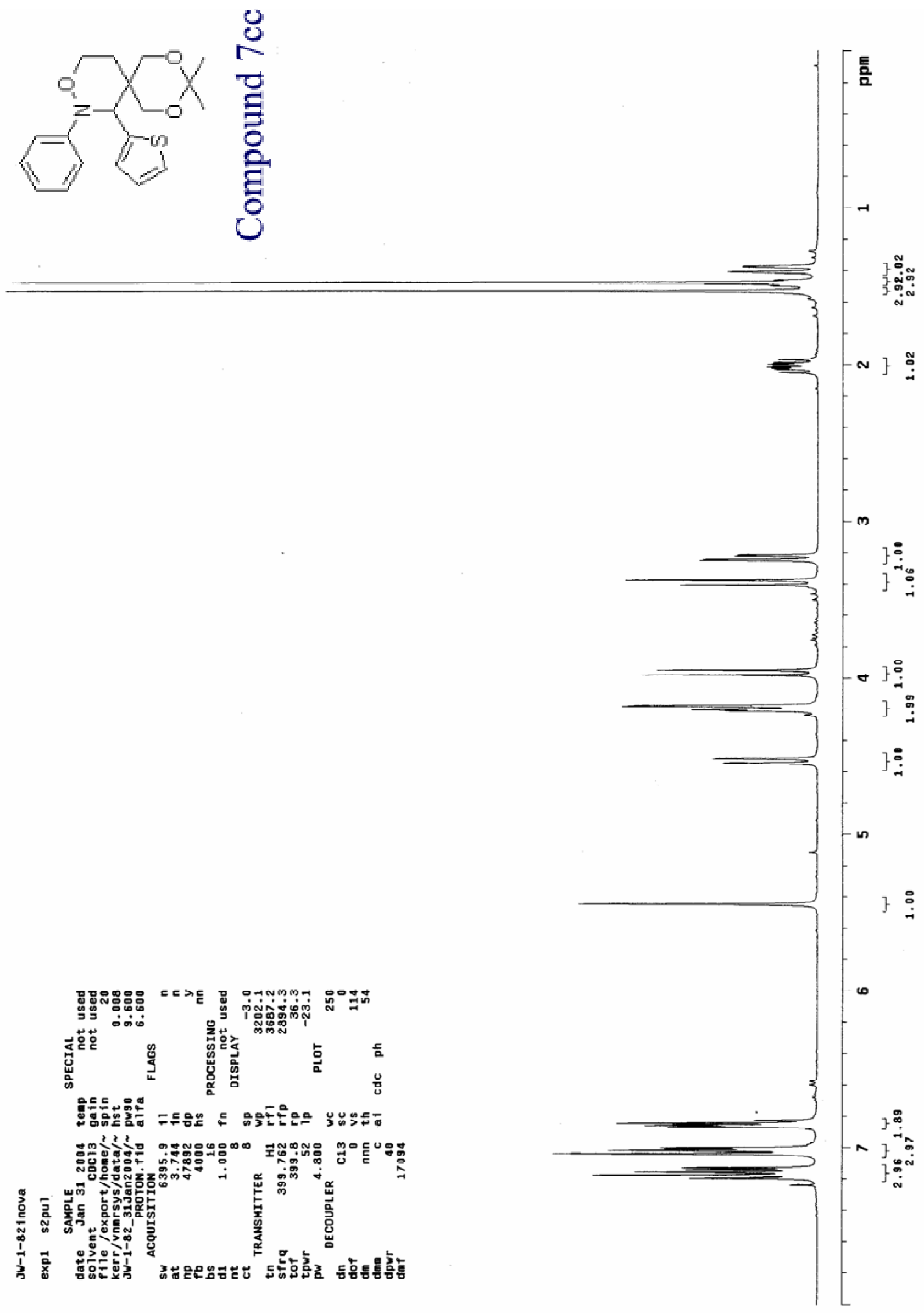

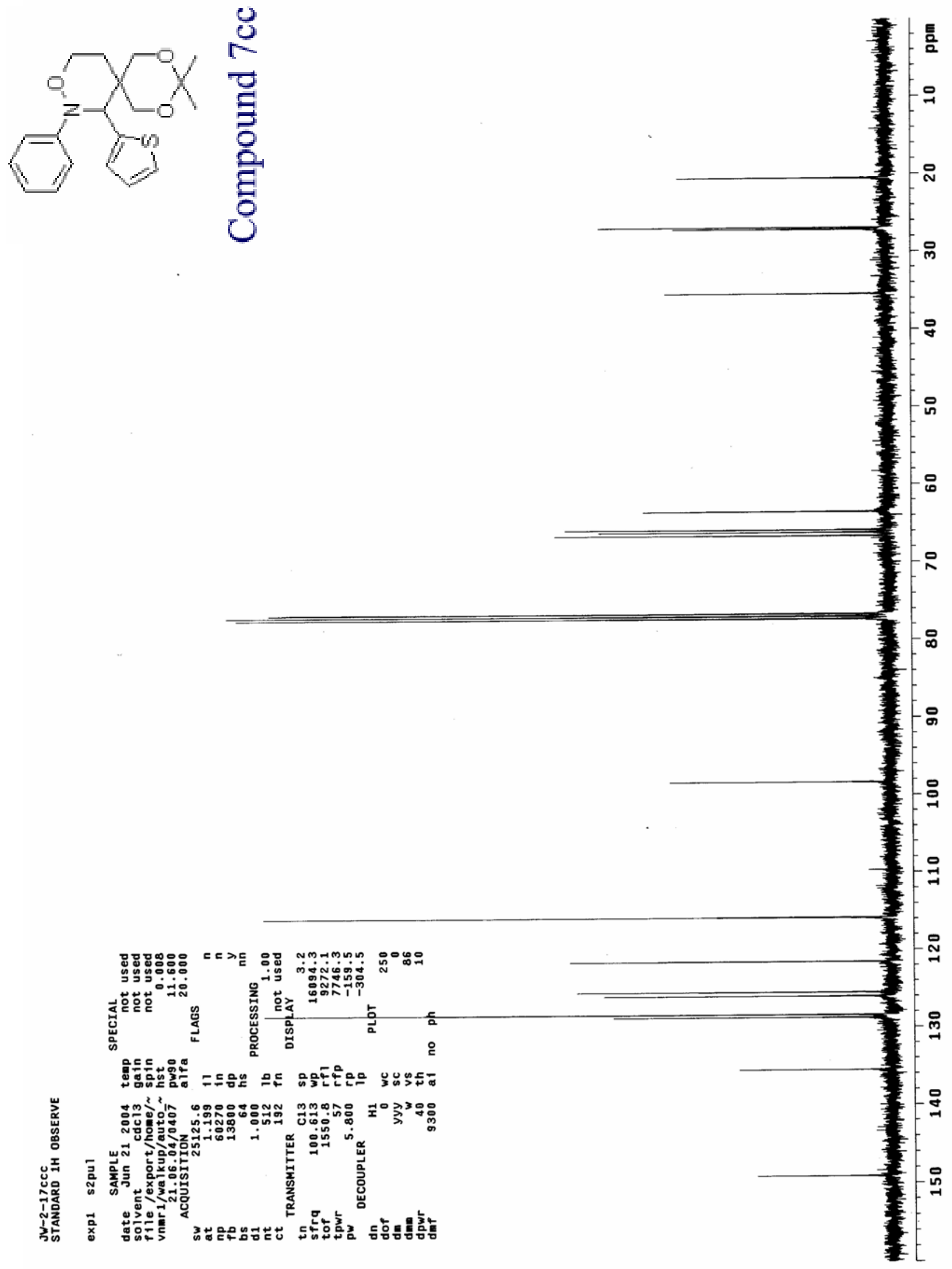

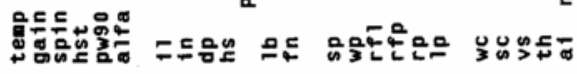

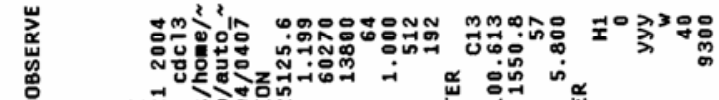

先 吾

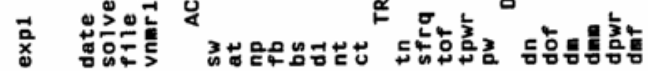




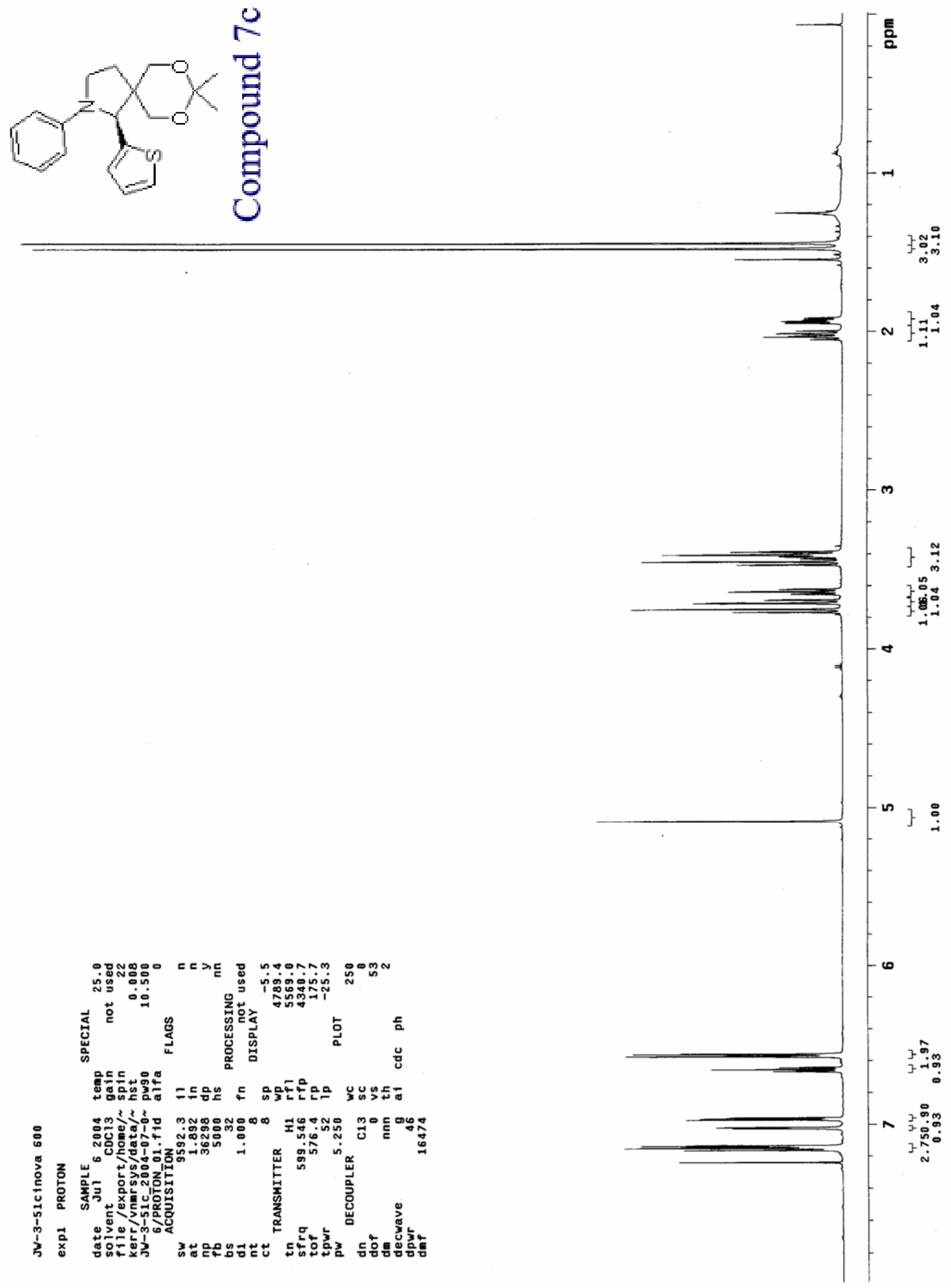


<smiles>CC1(C)OCC2(CC[Z](c3ccccc3)[C@H]2c2ccco2)CO1</smiles>

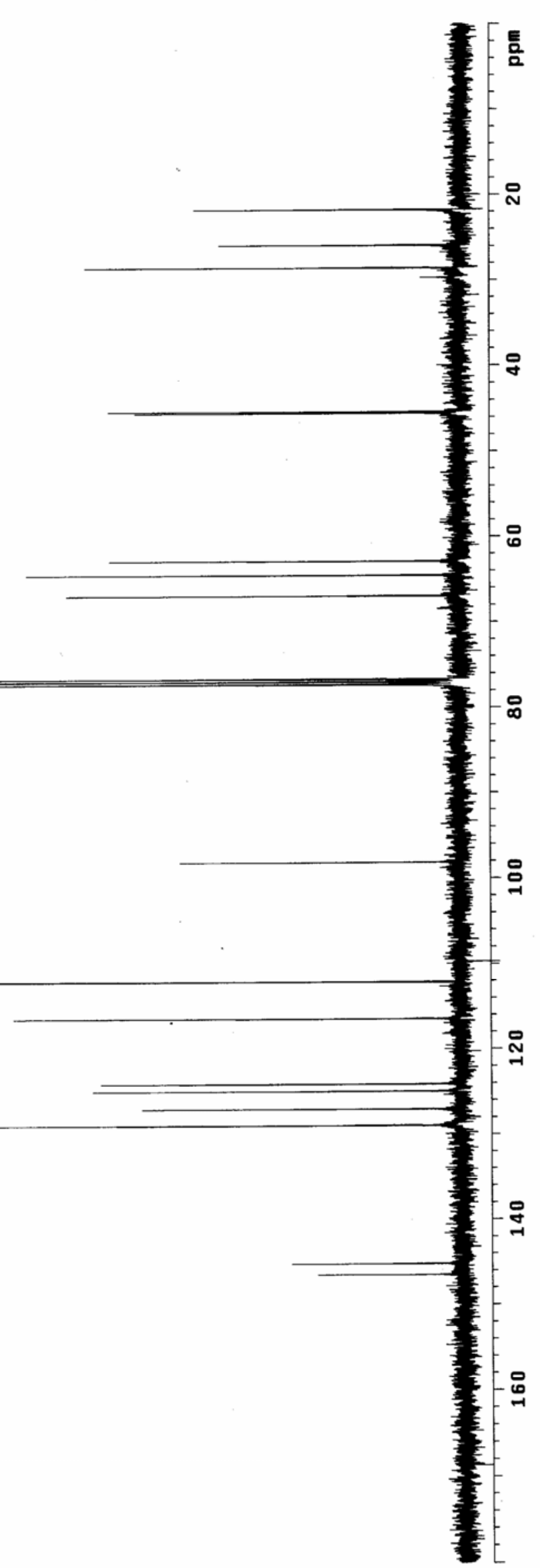

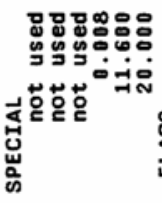

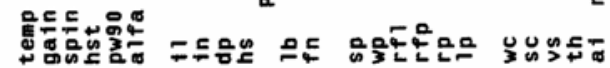

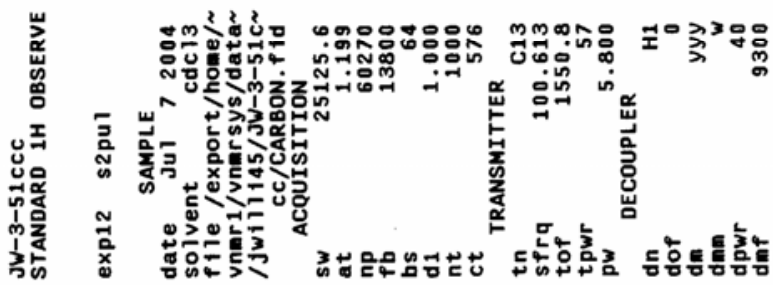



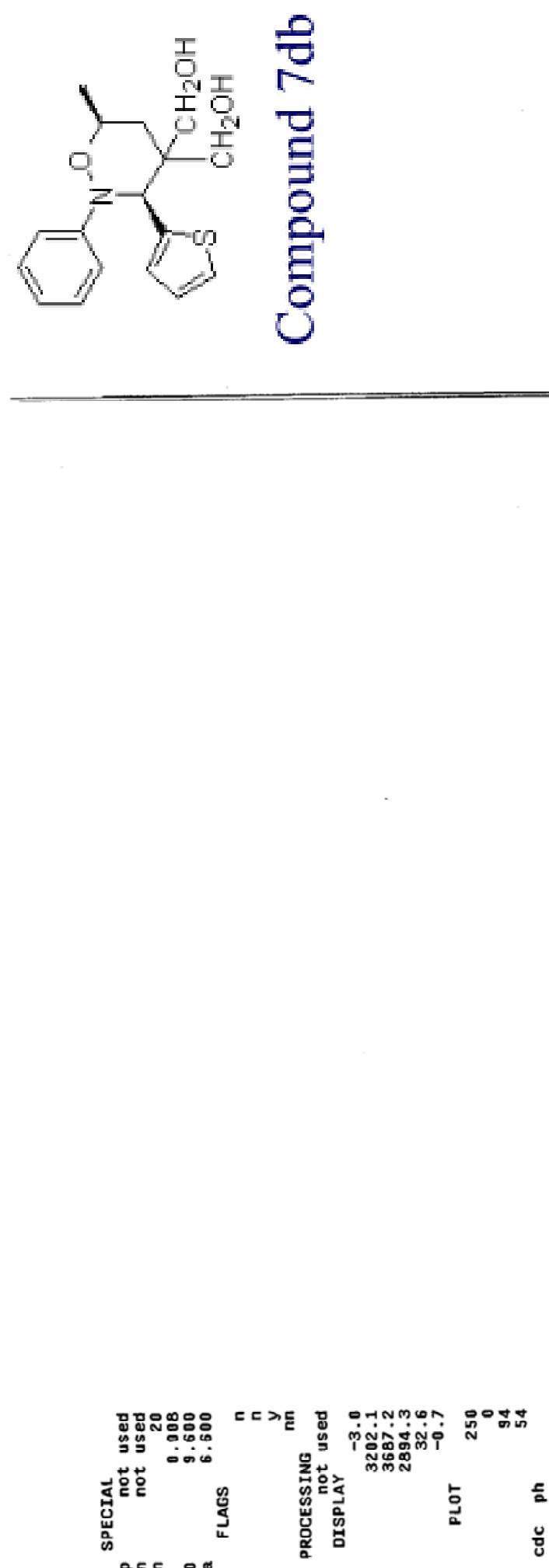

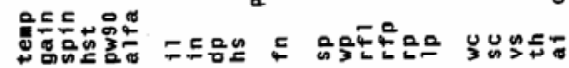

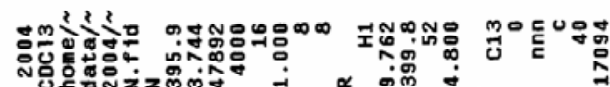

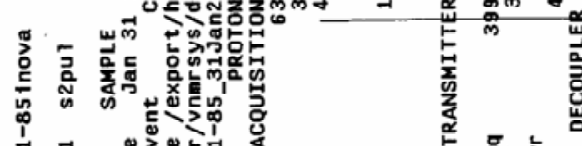

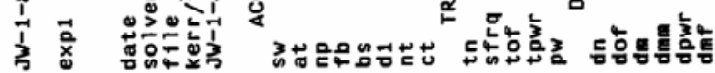

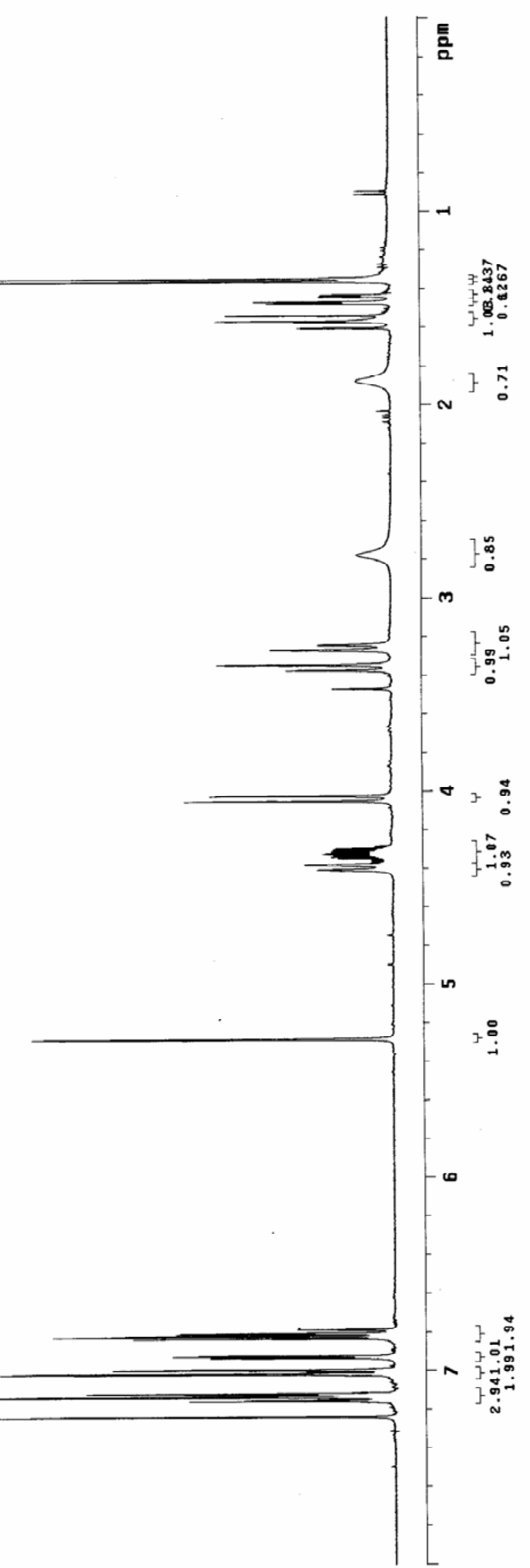



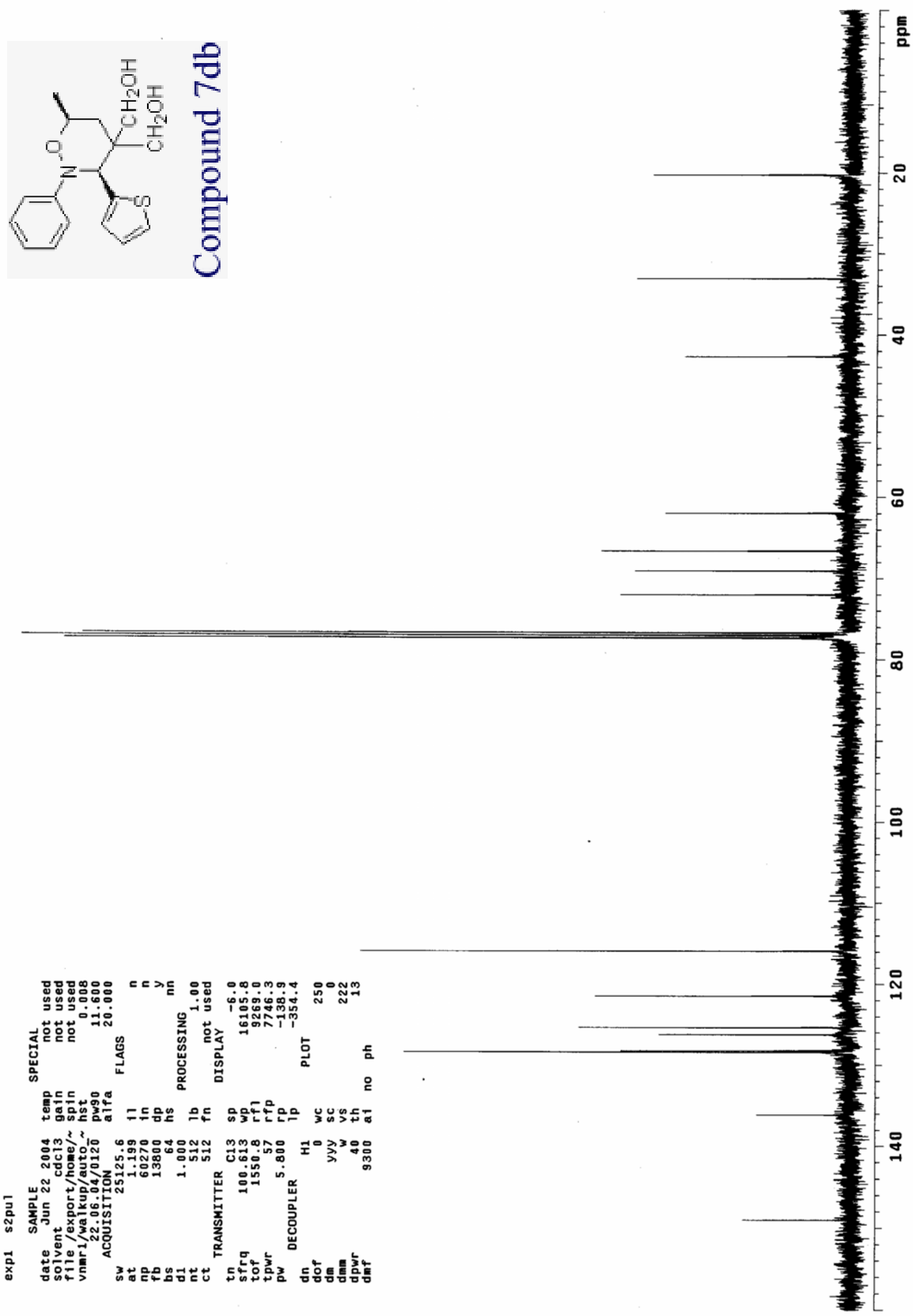

= cre 요

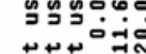

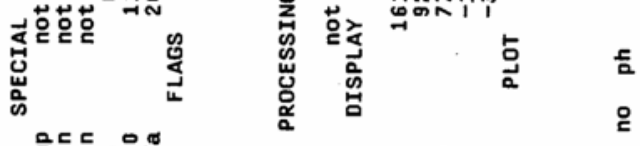

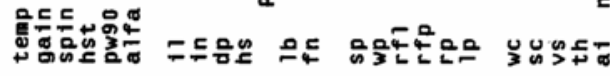

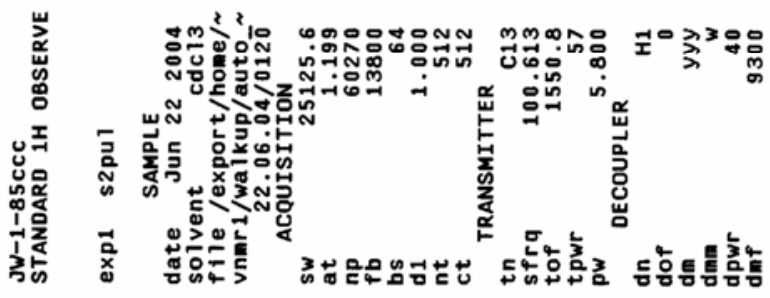




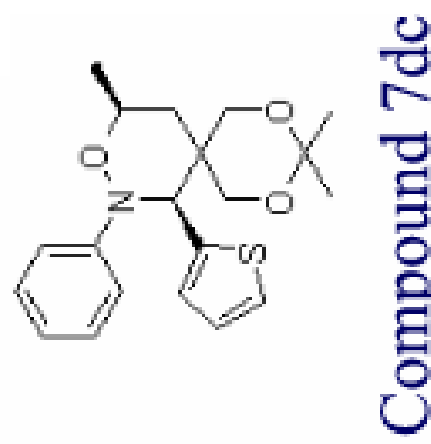

틀

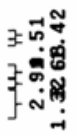

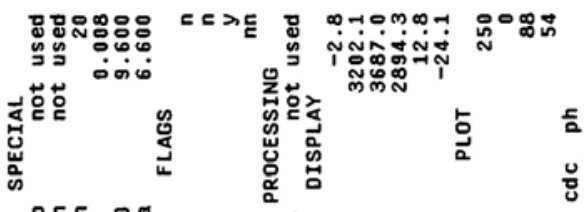

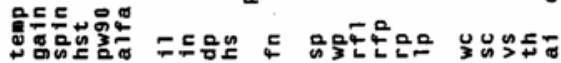
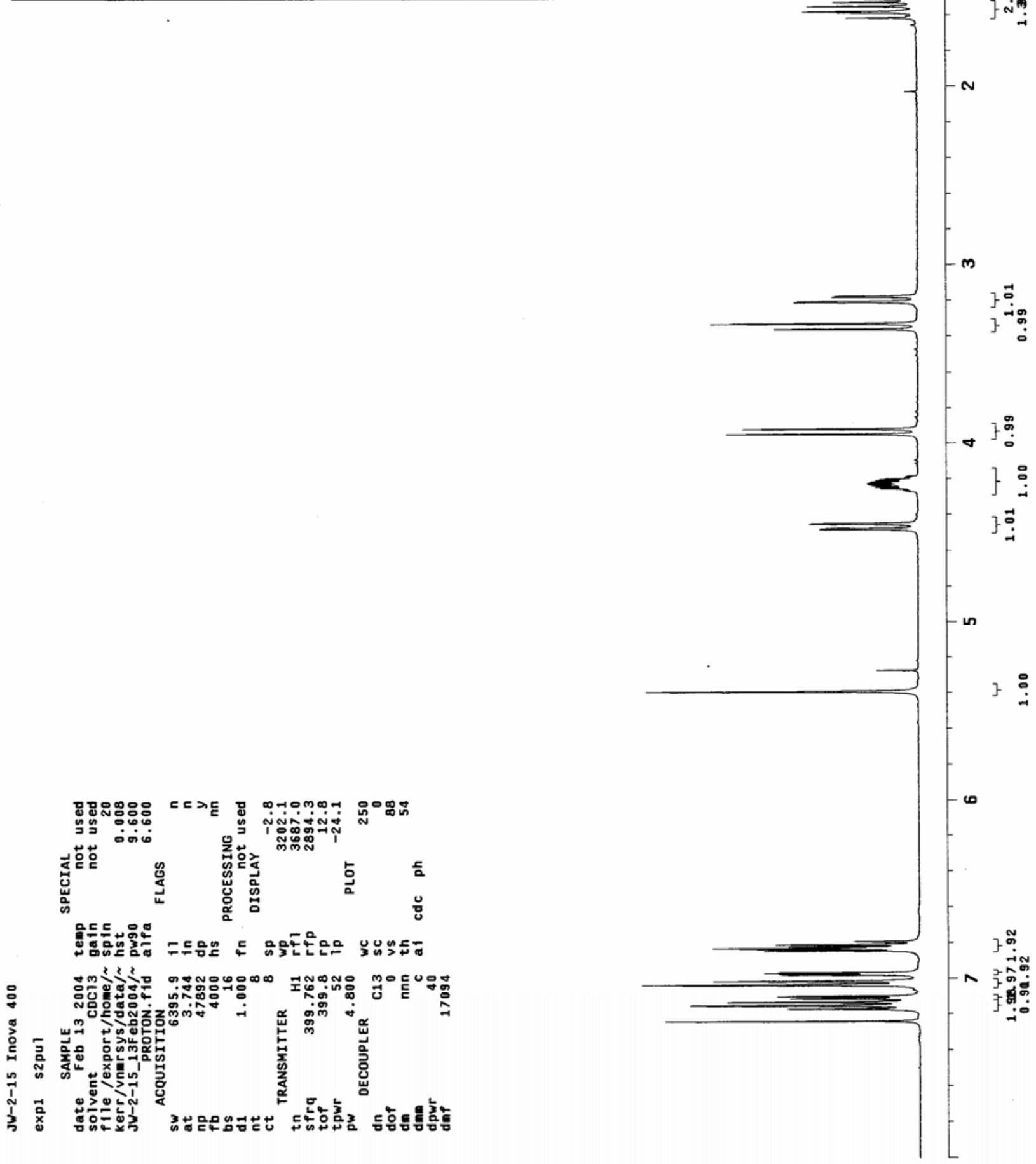


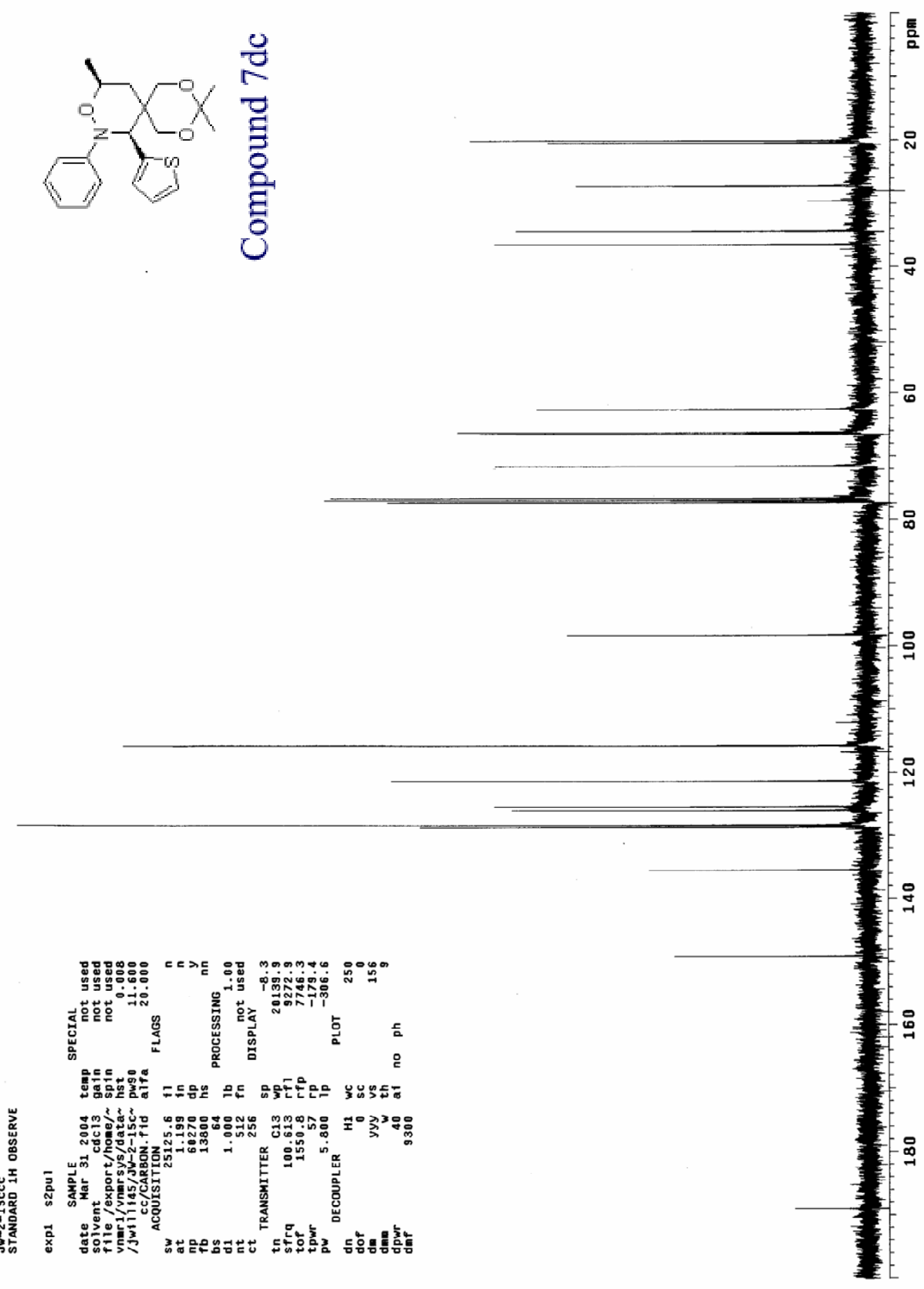




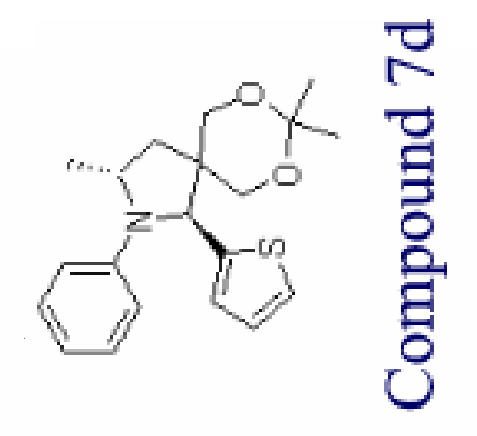

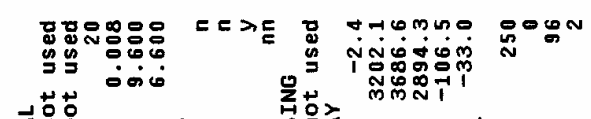

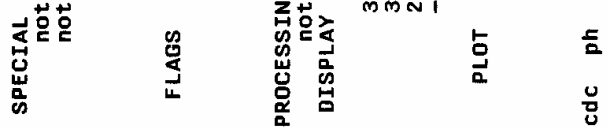

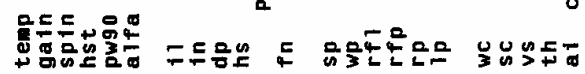

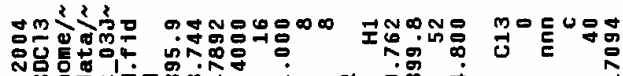
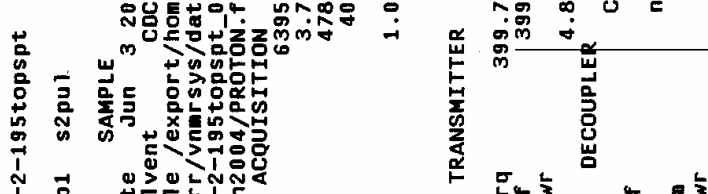

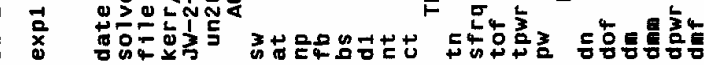



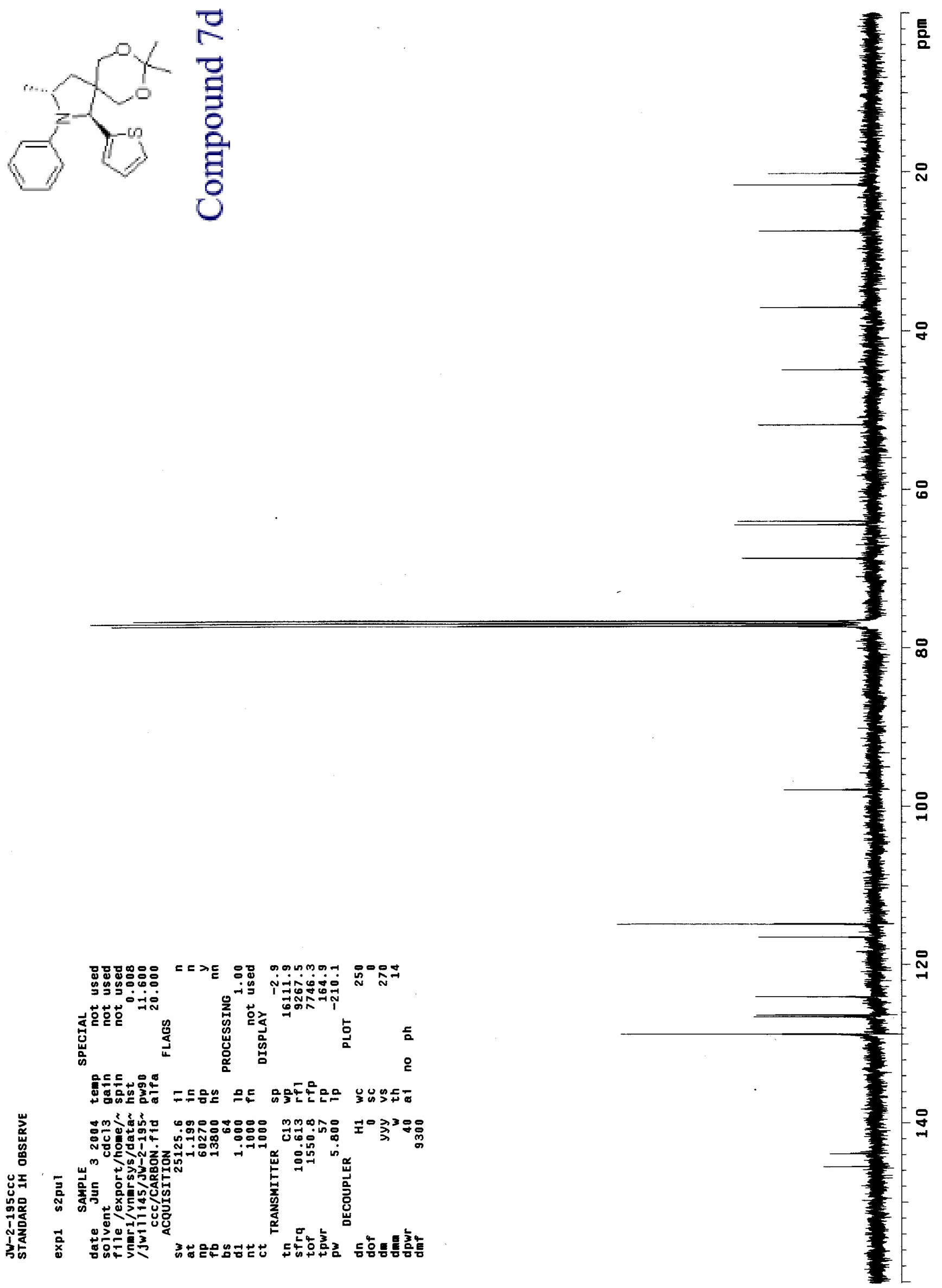


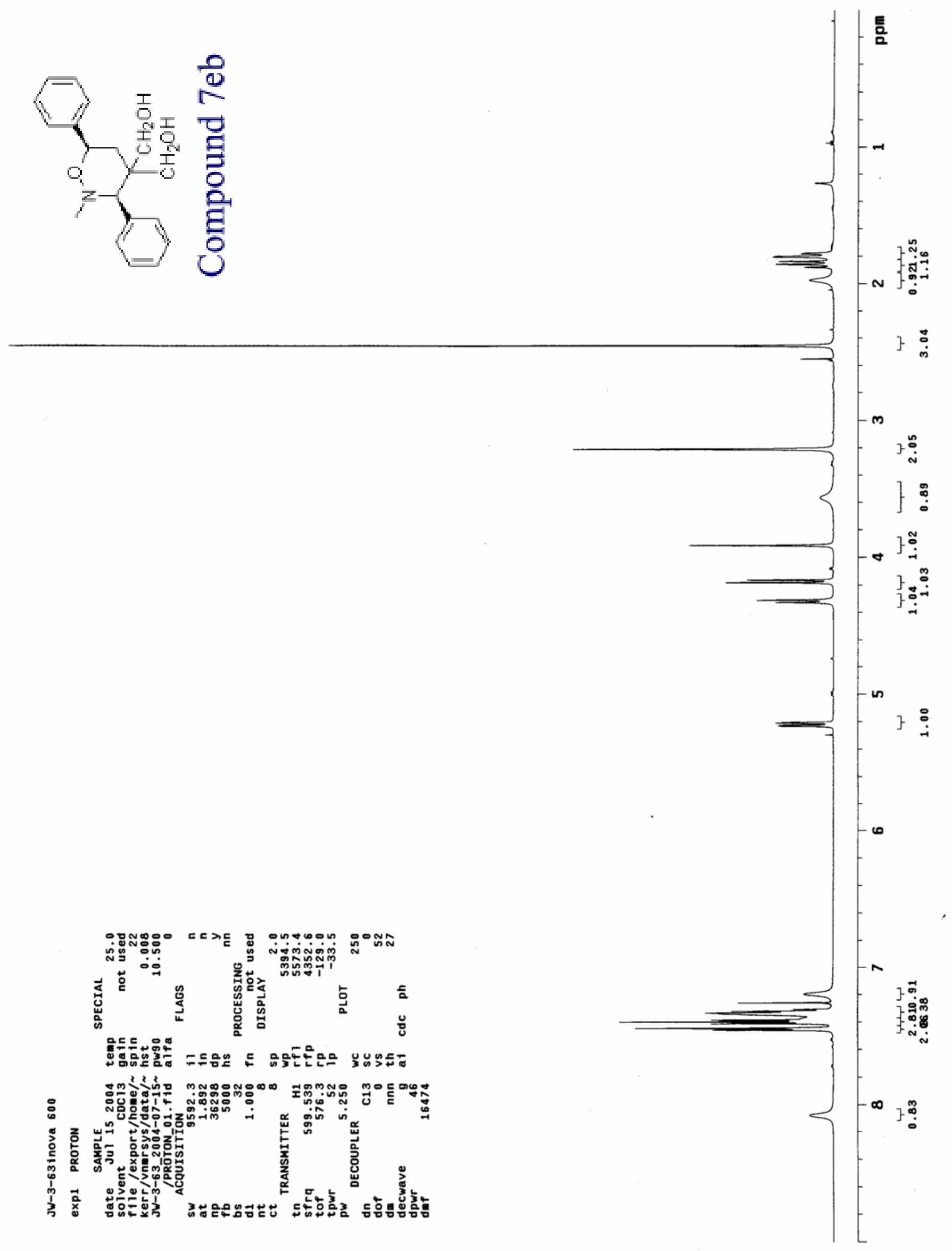



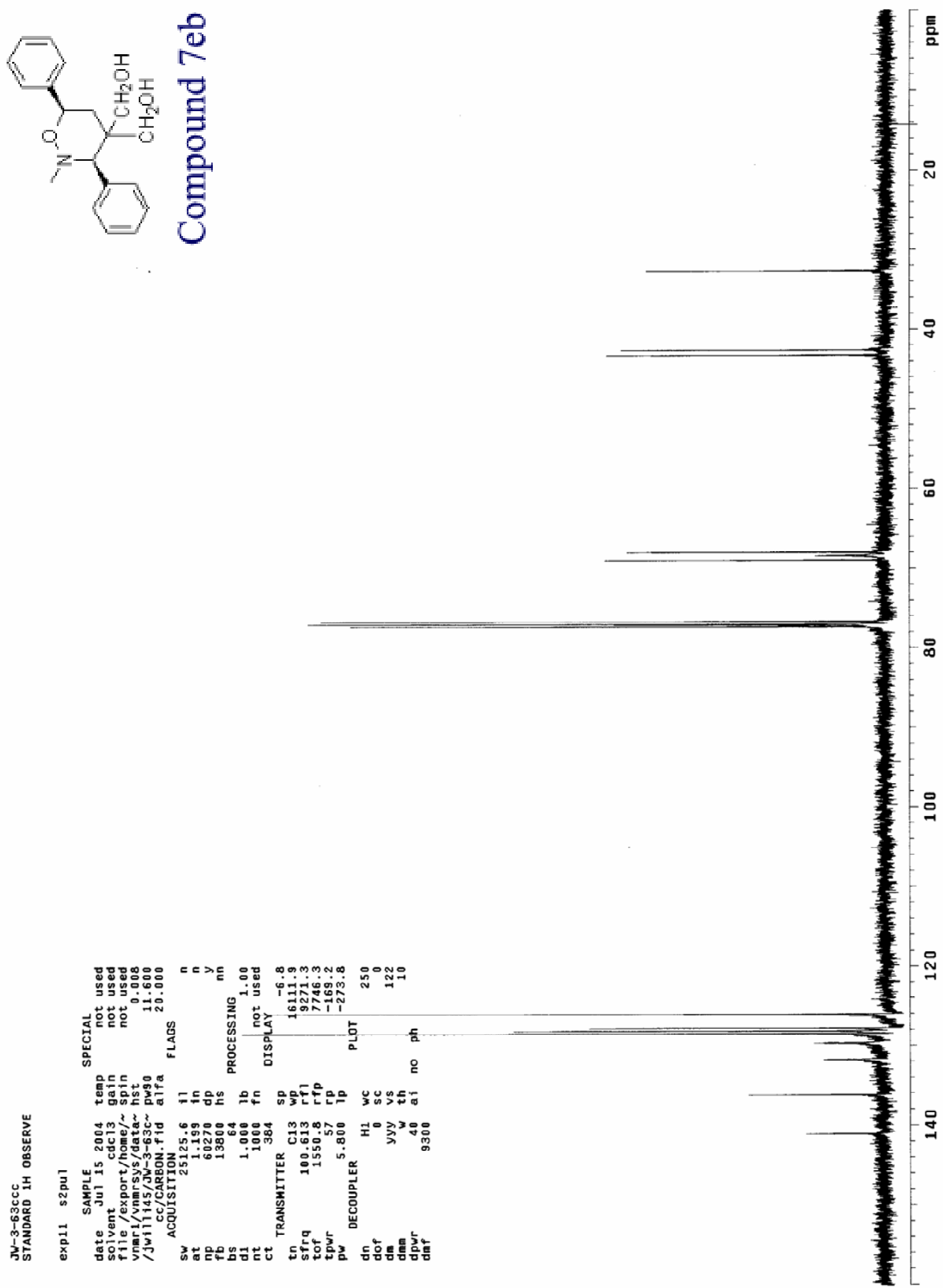

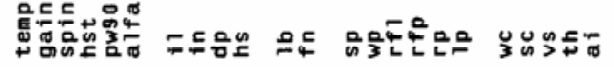

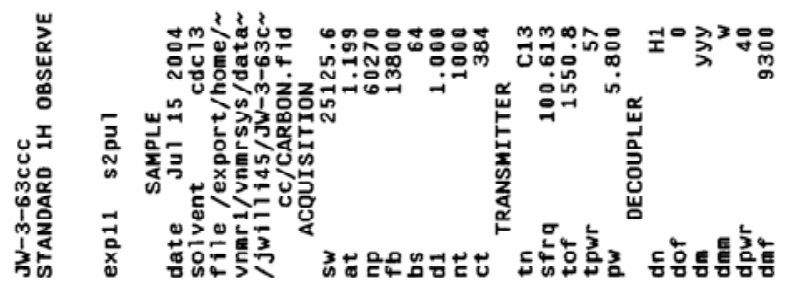




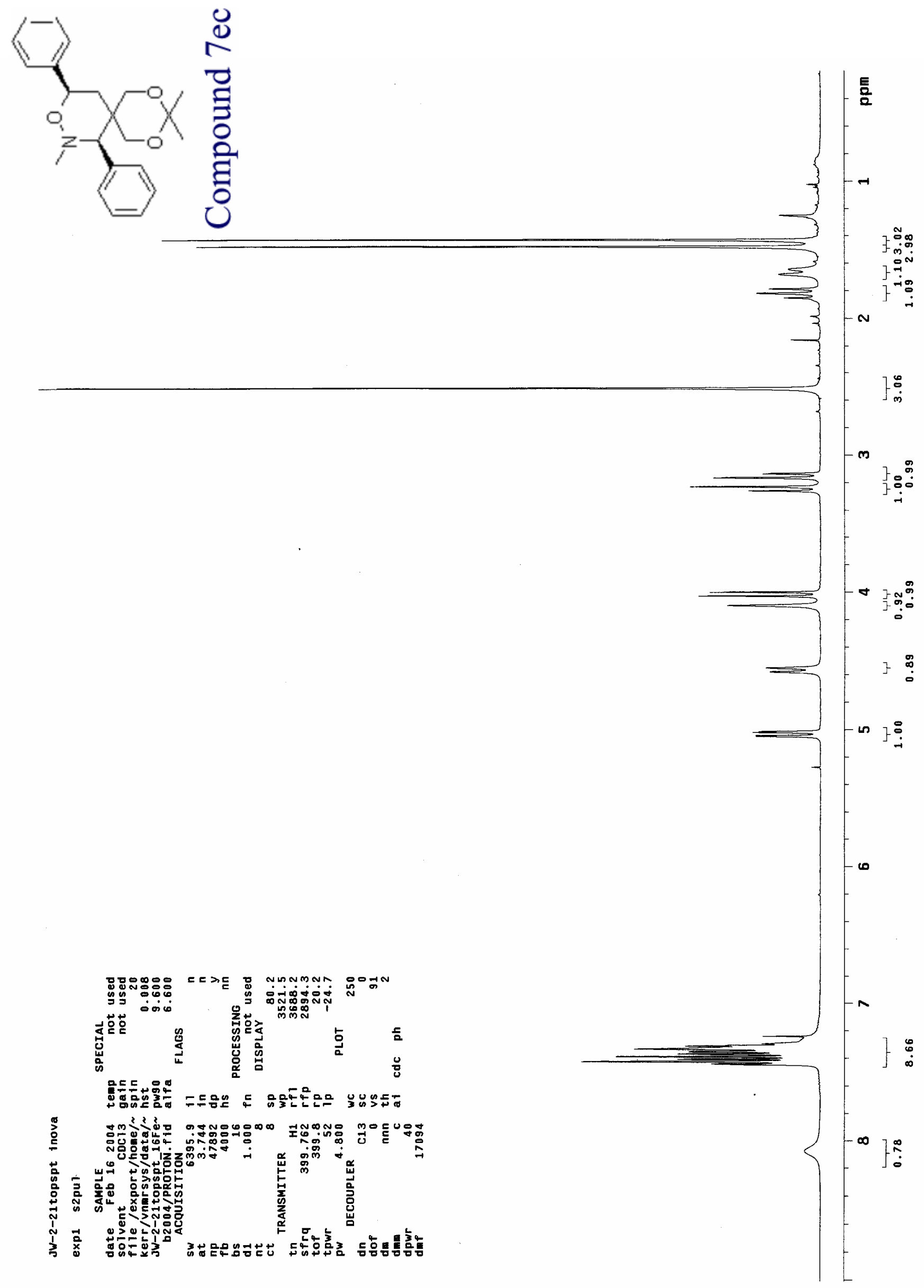



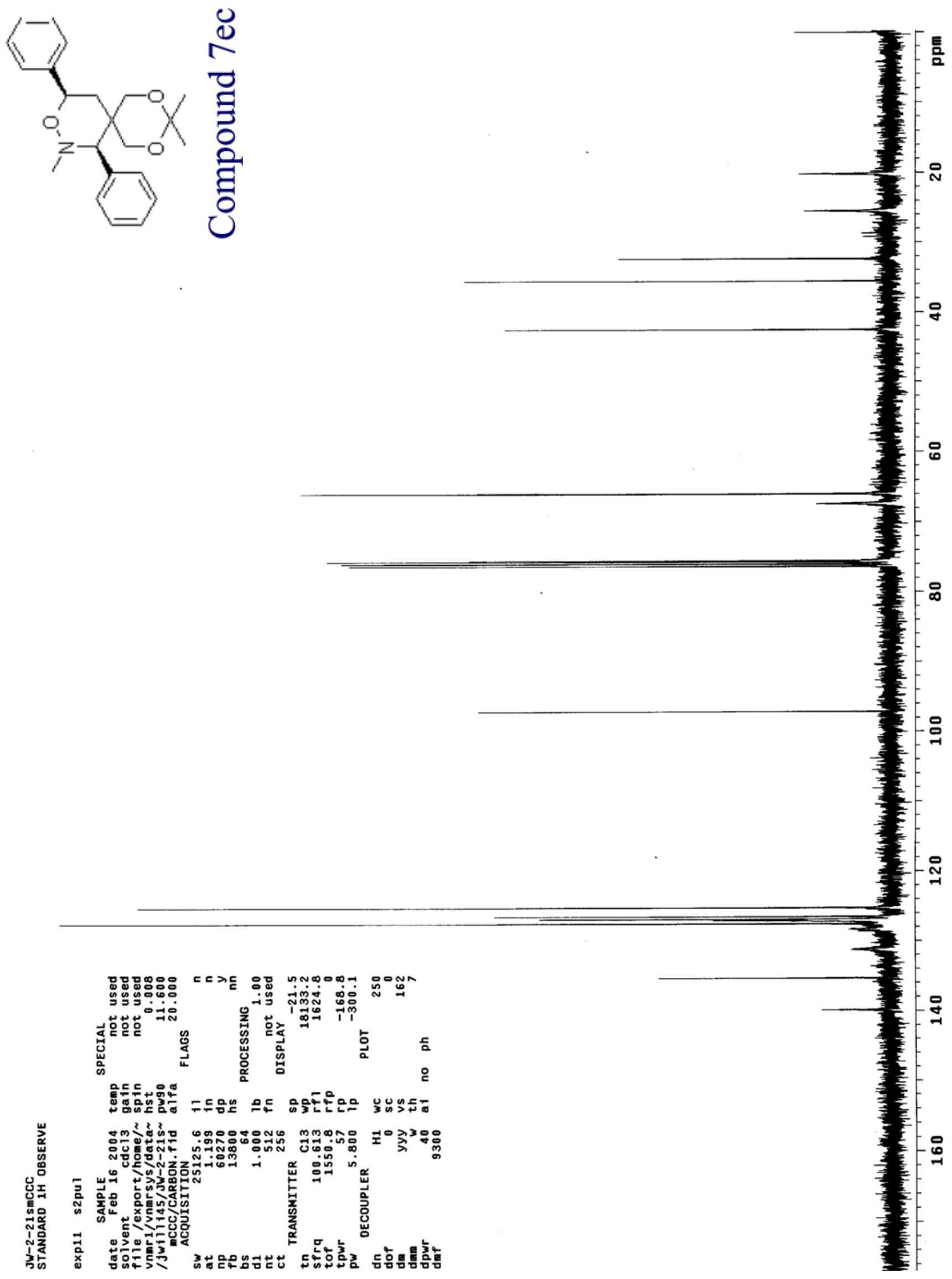


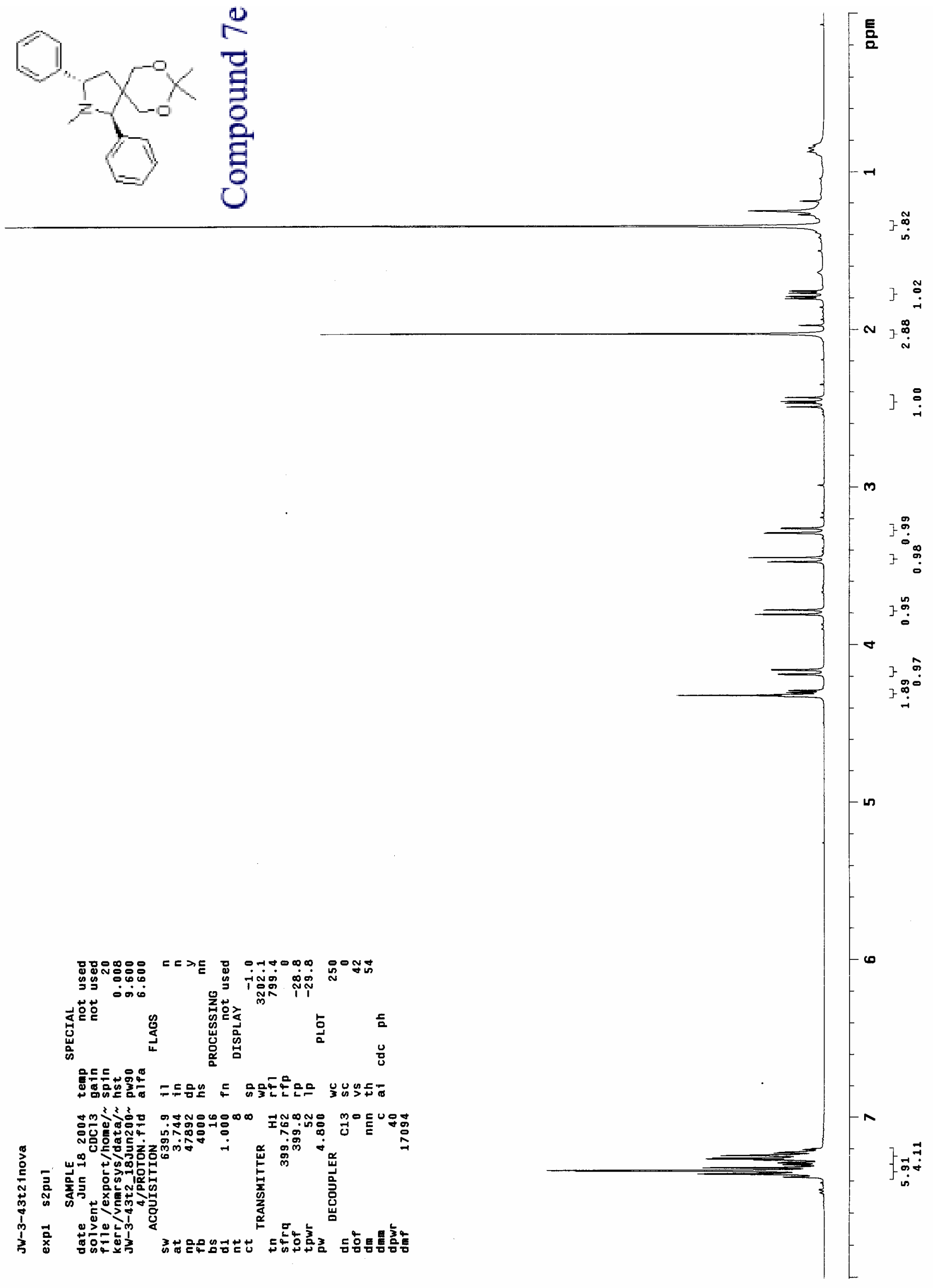



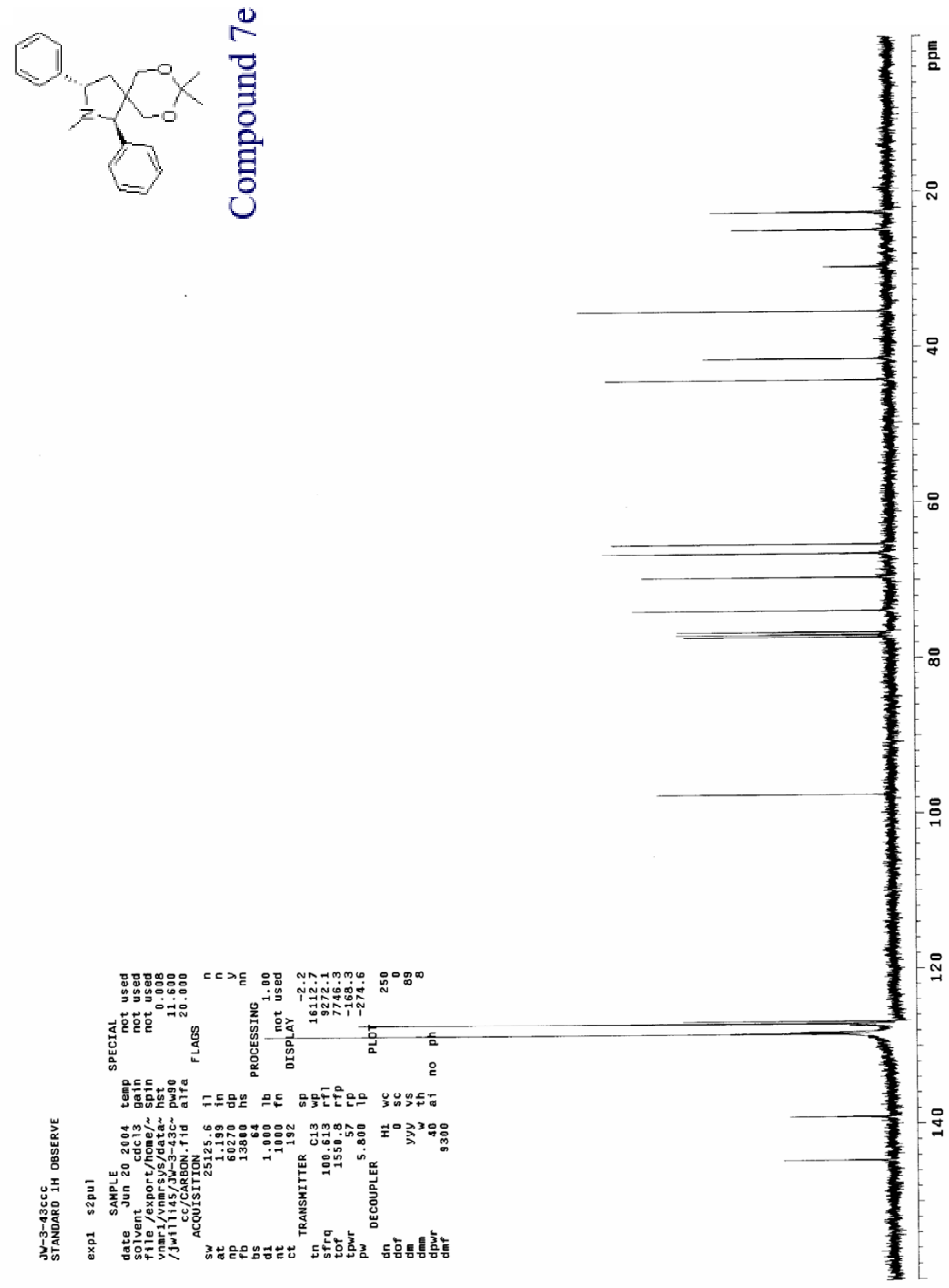


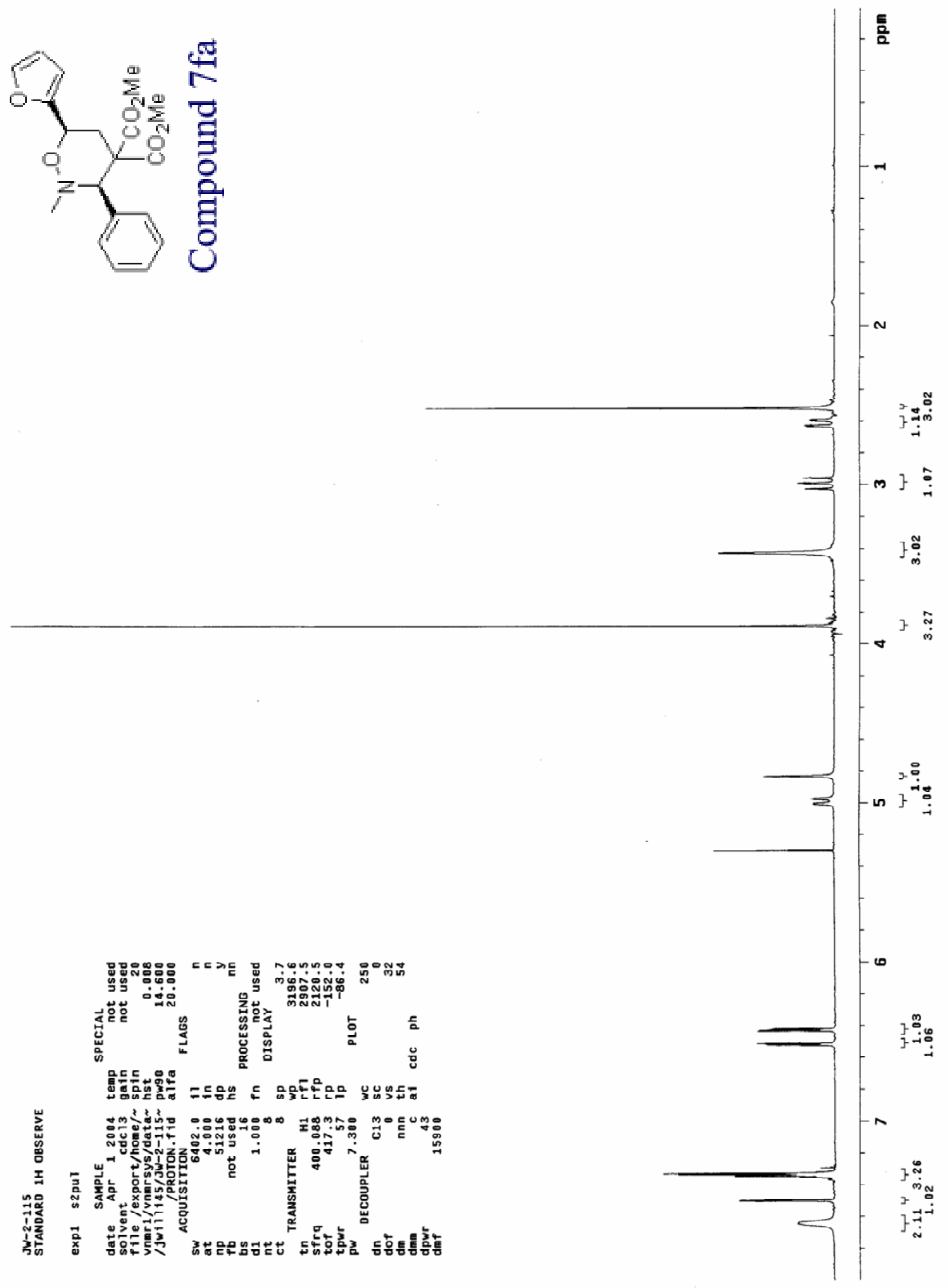



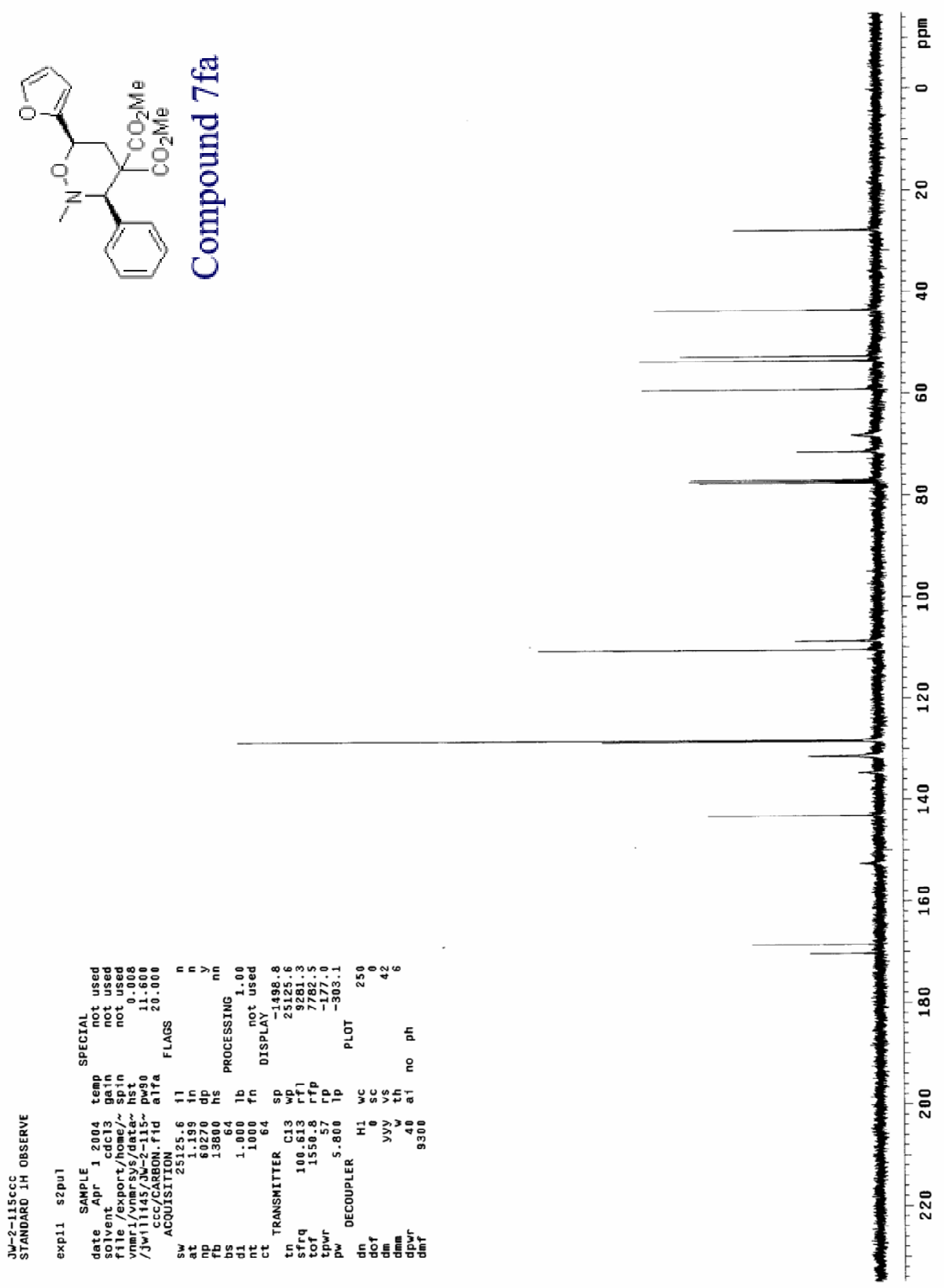

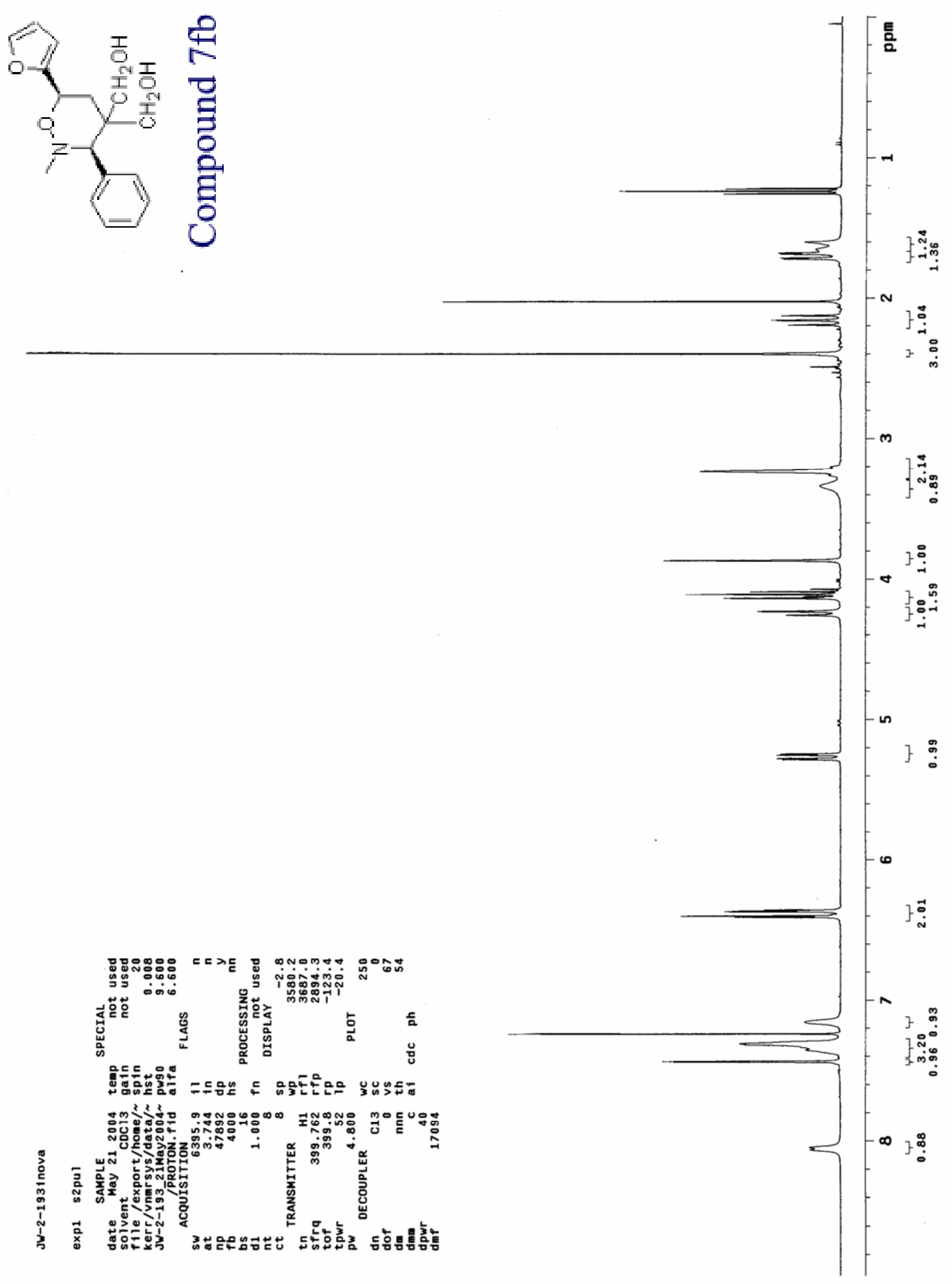

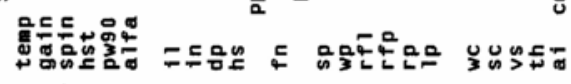

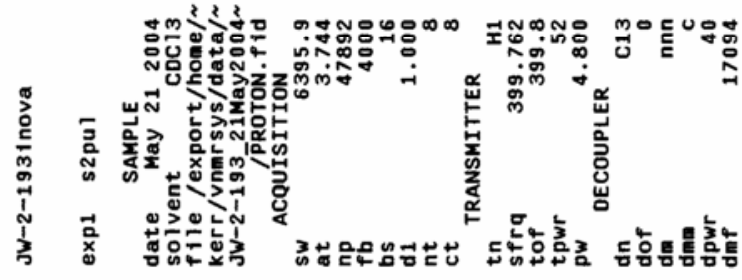




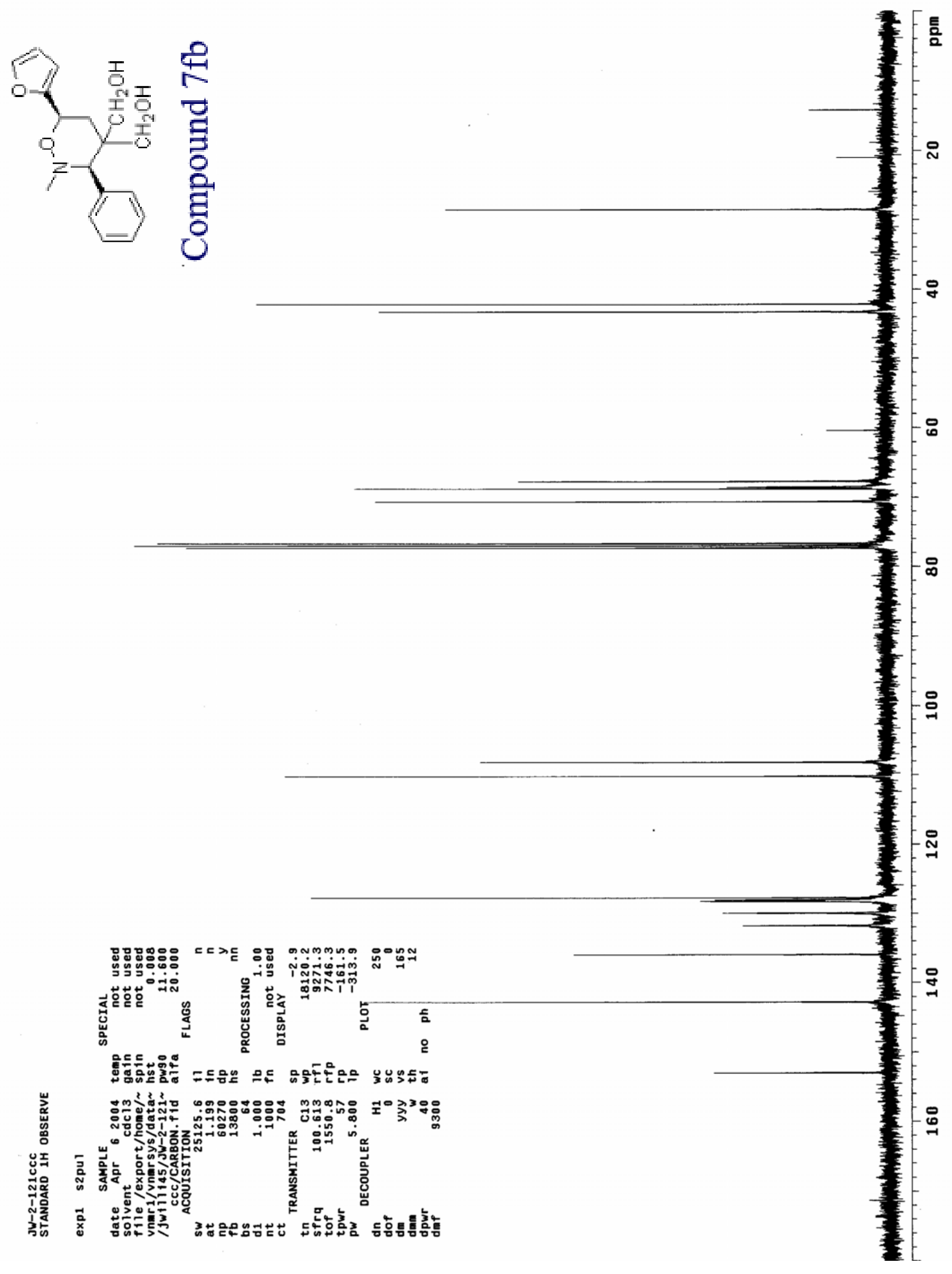



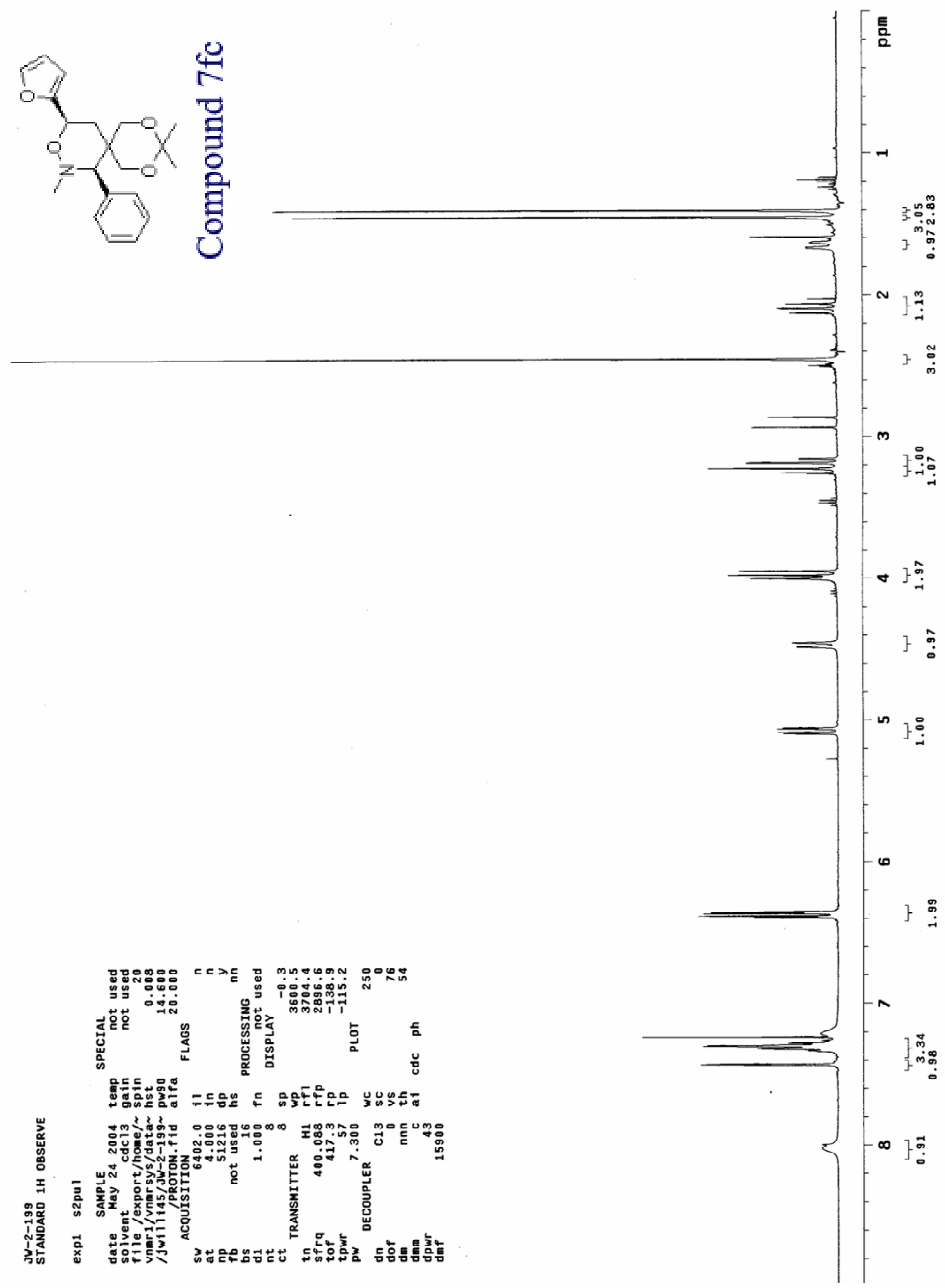

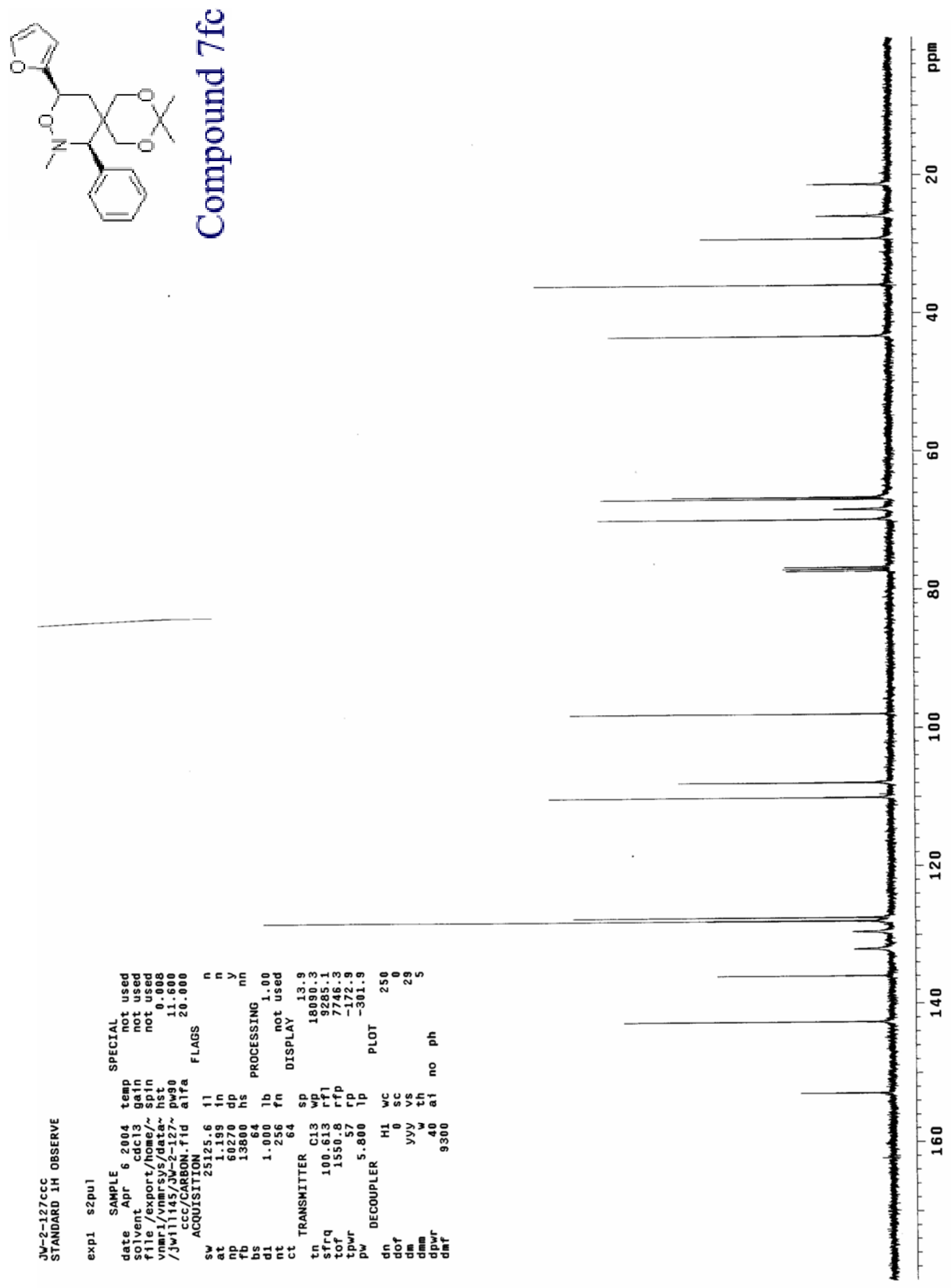


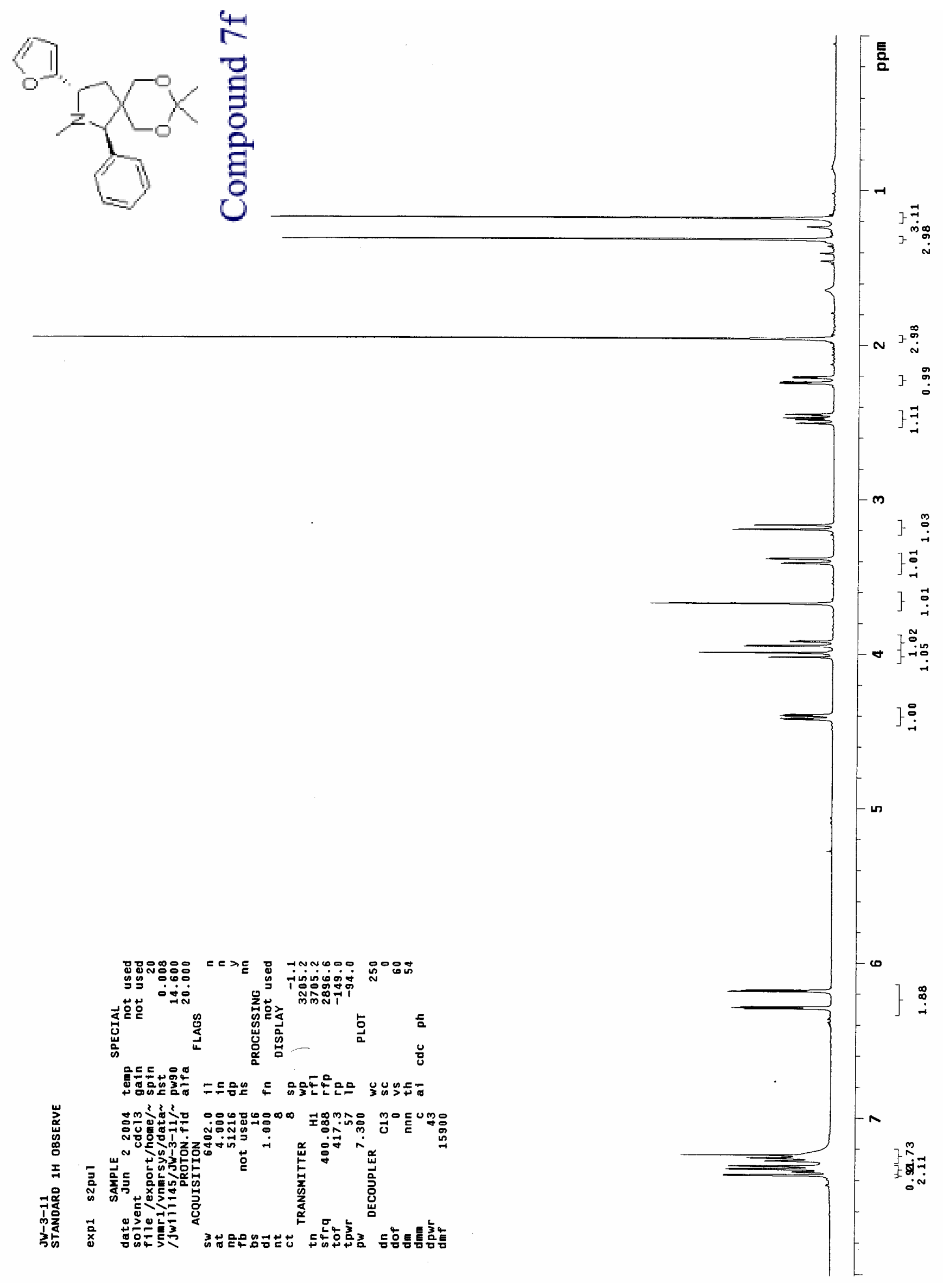



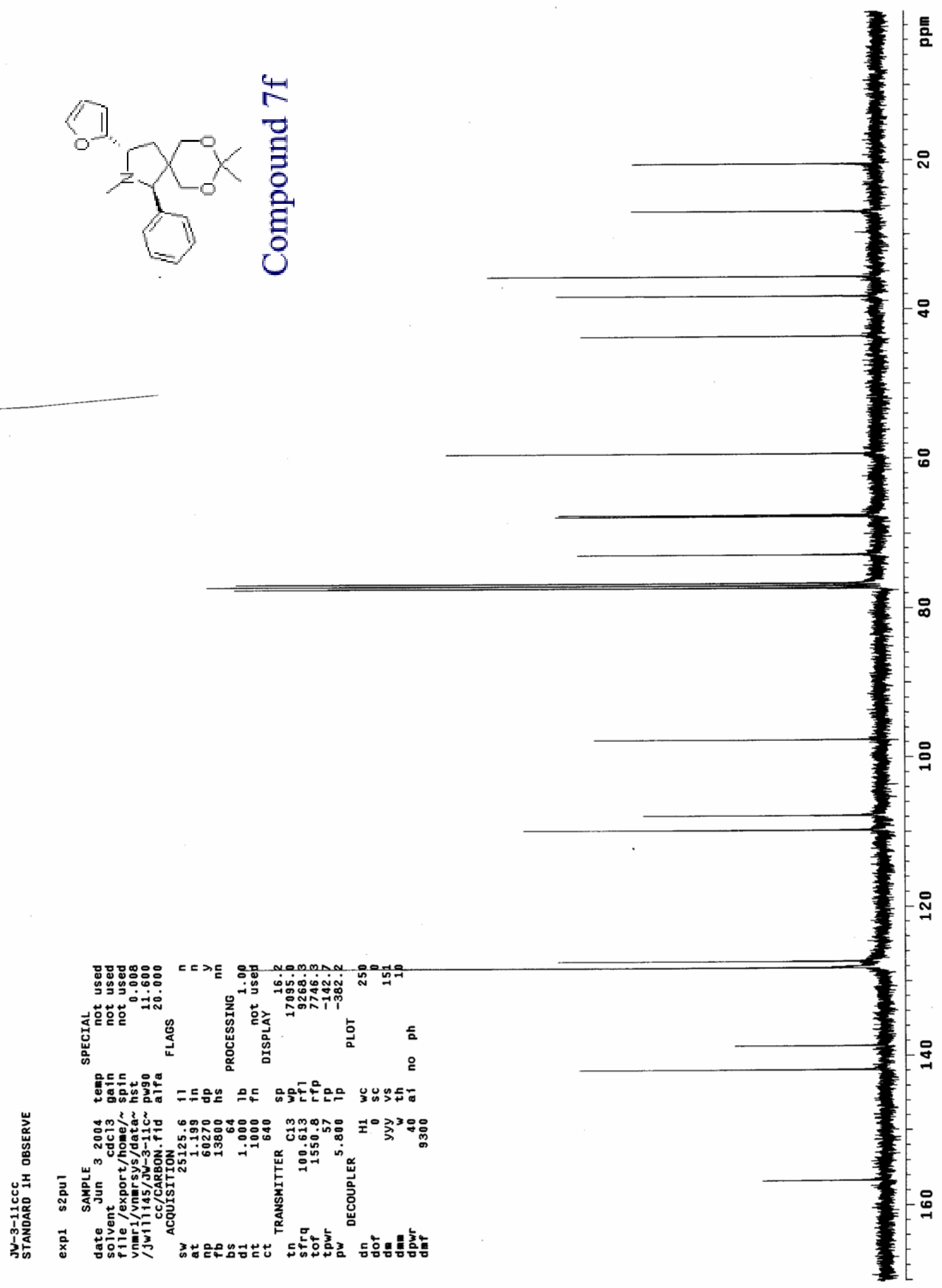

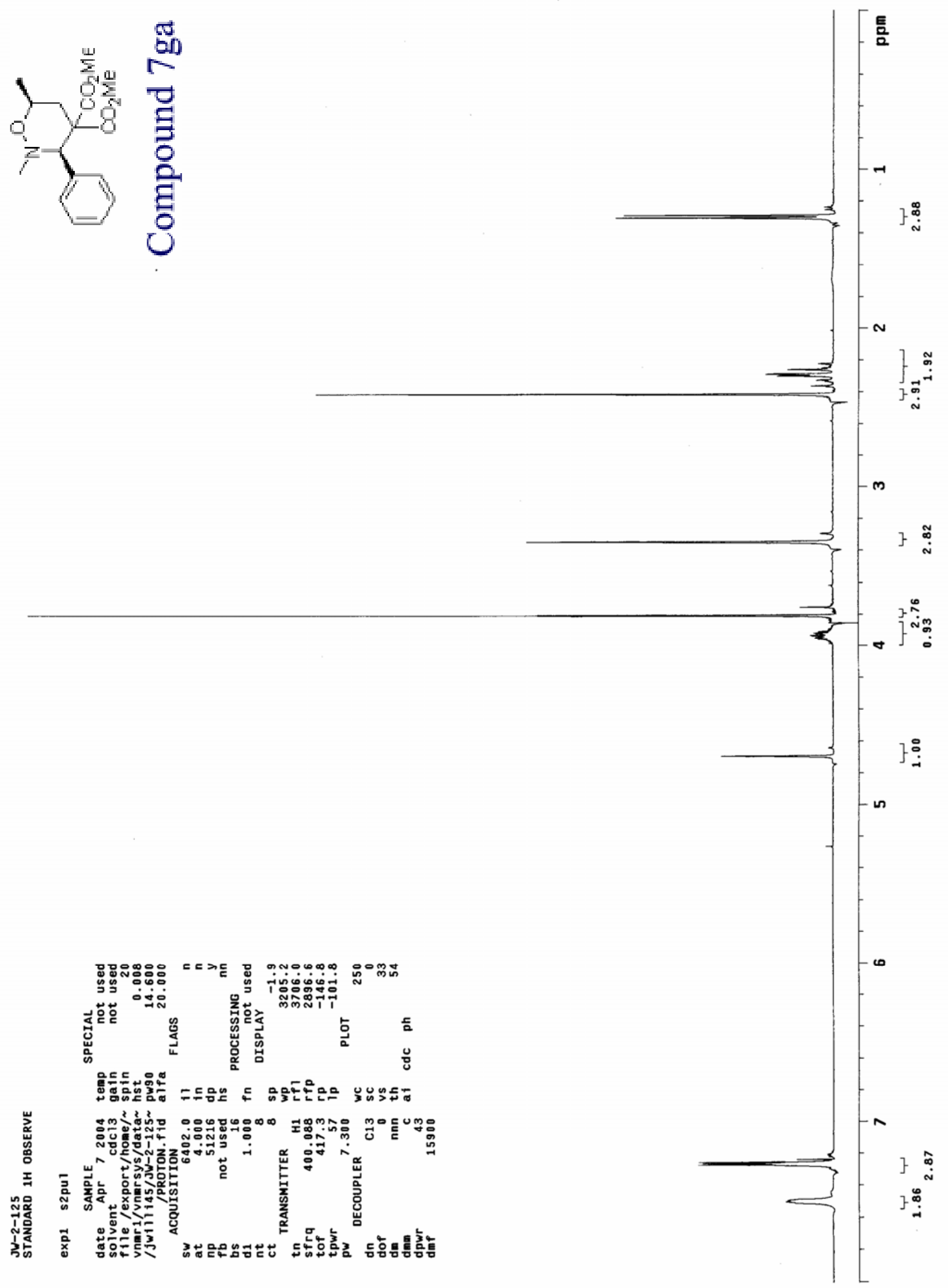

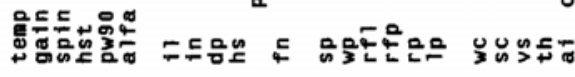

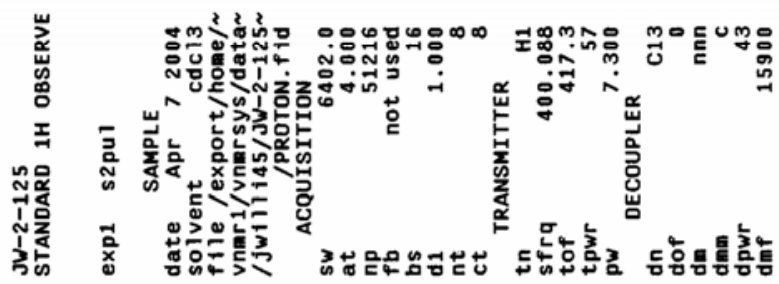



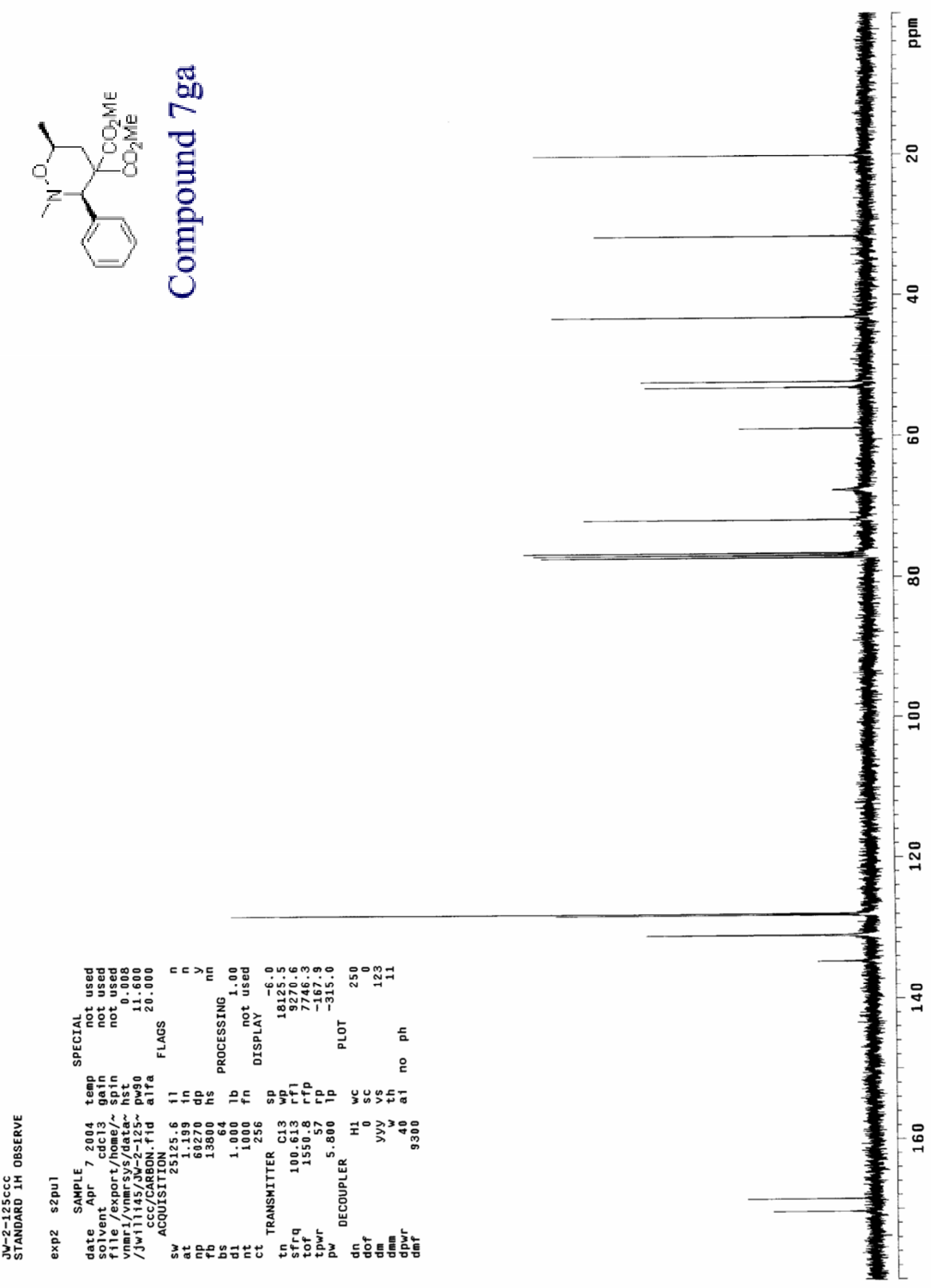

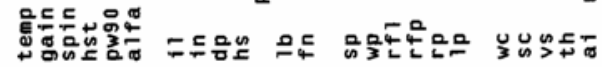

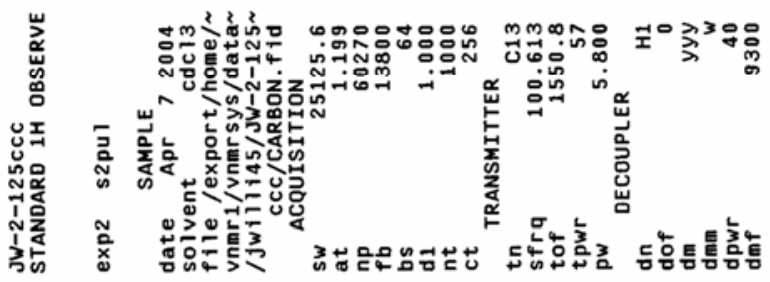




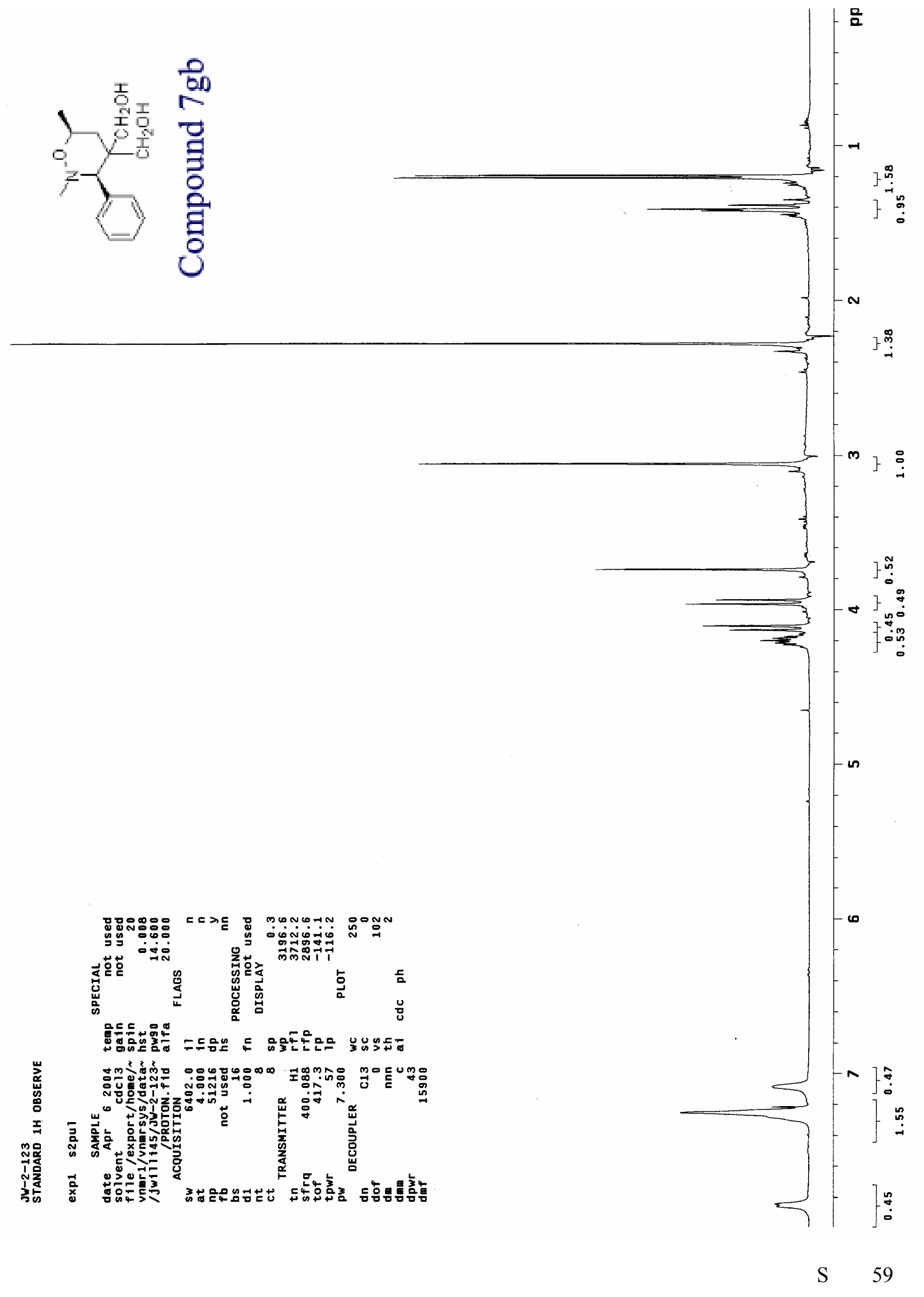



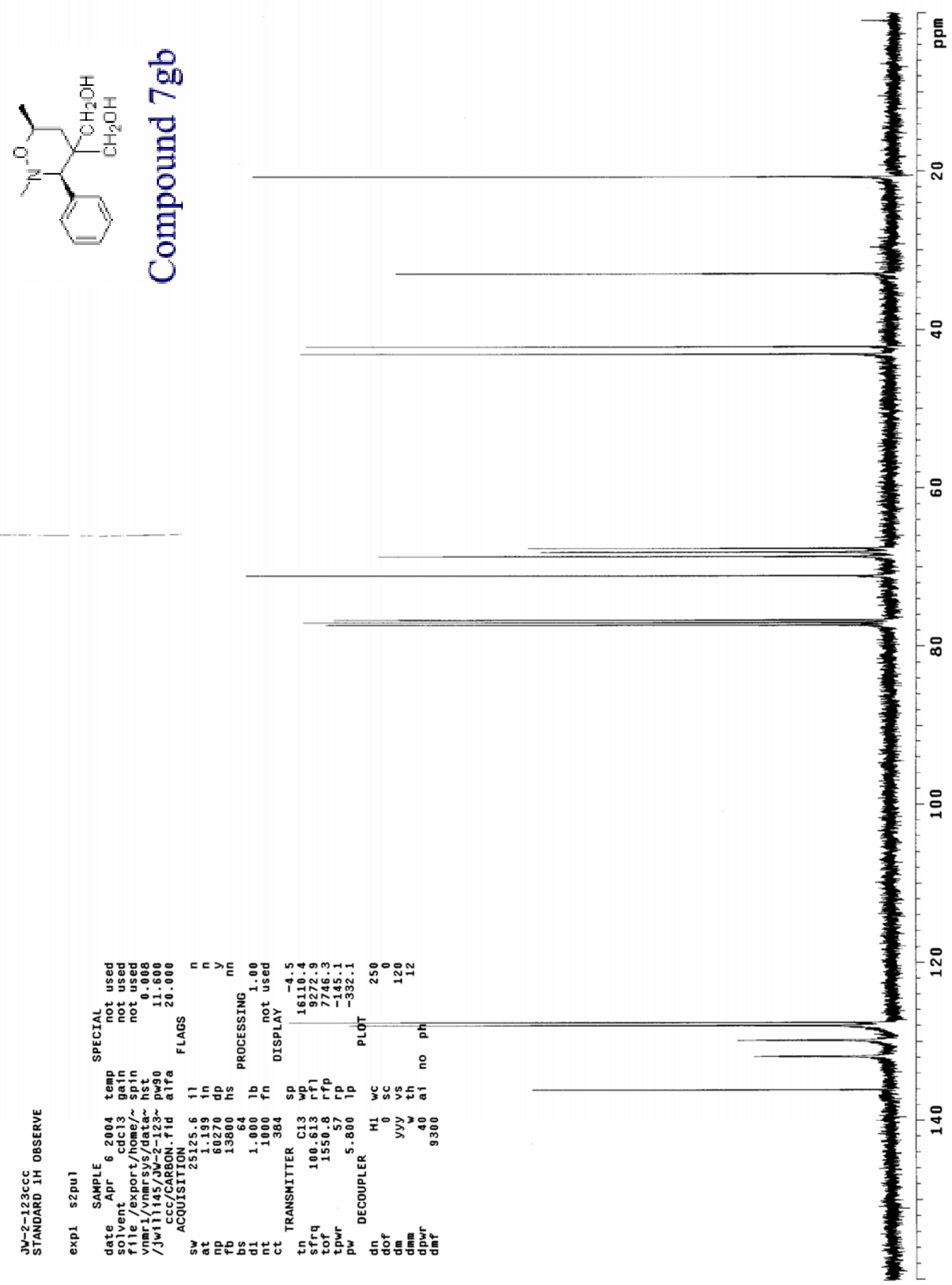

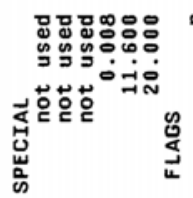

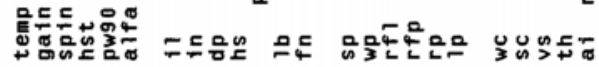

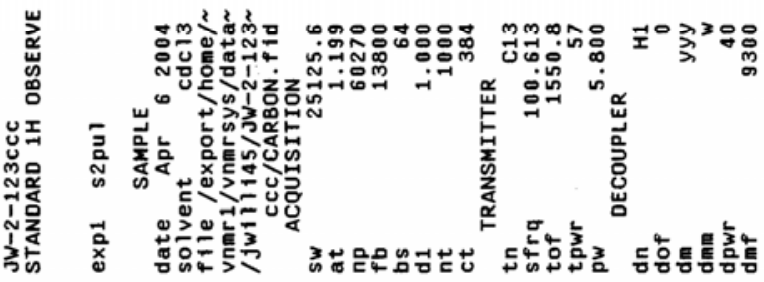


\=
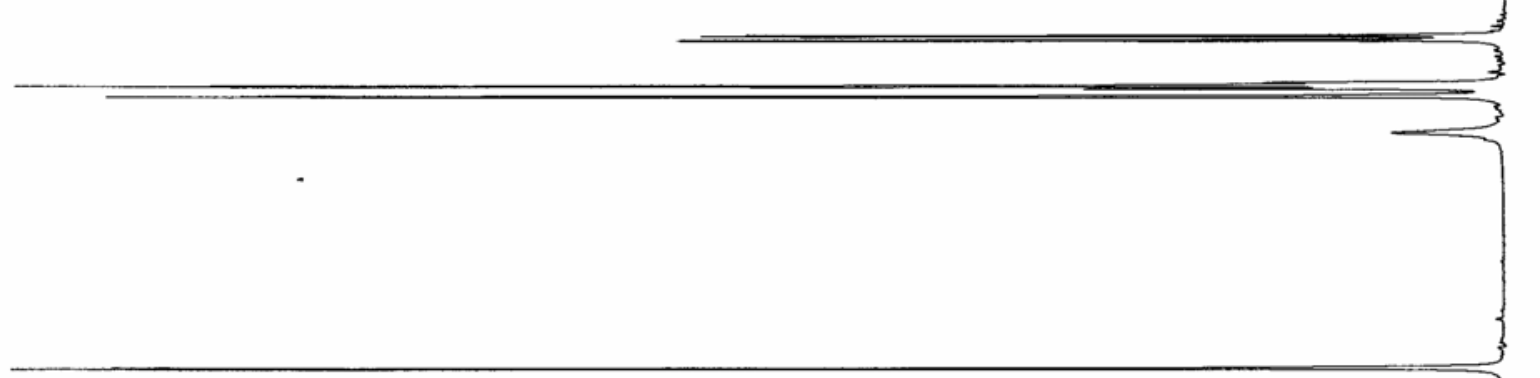

ᄀ

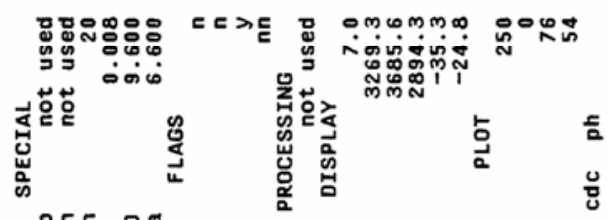

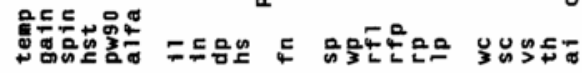
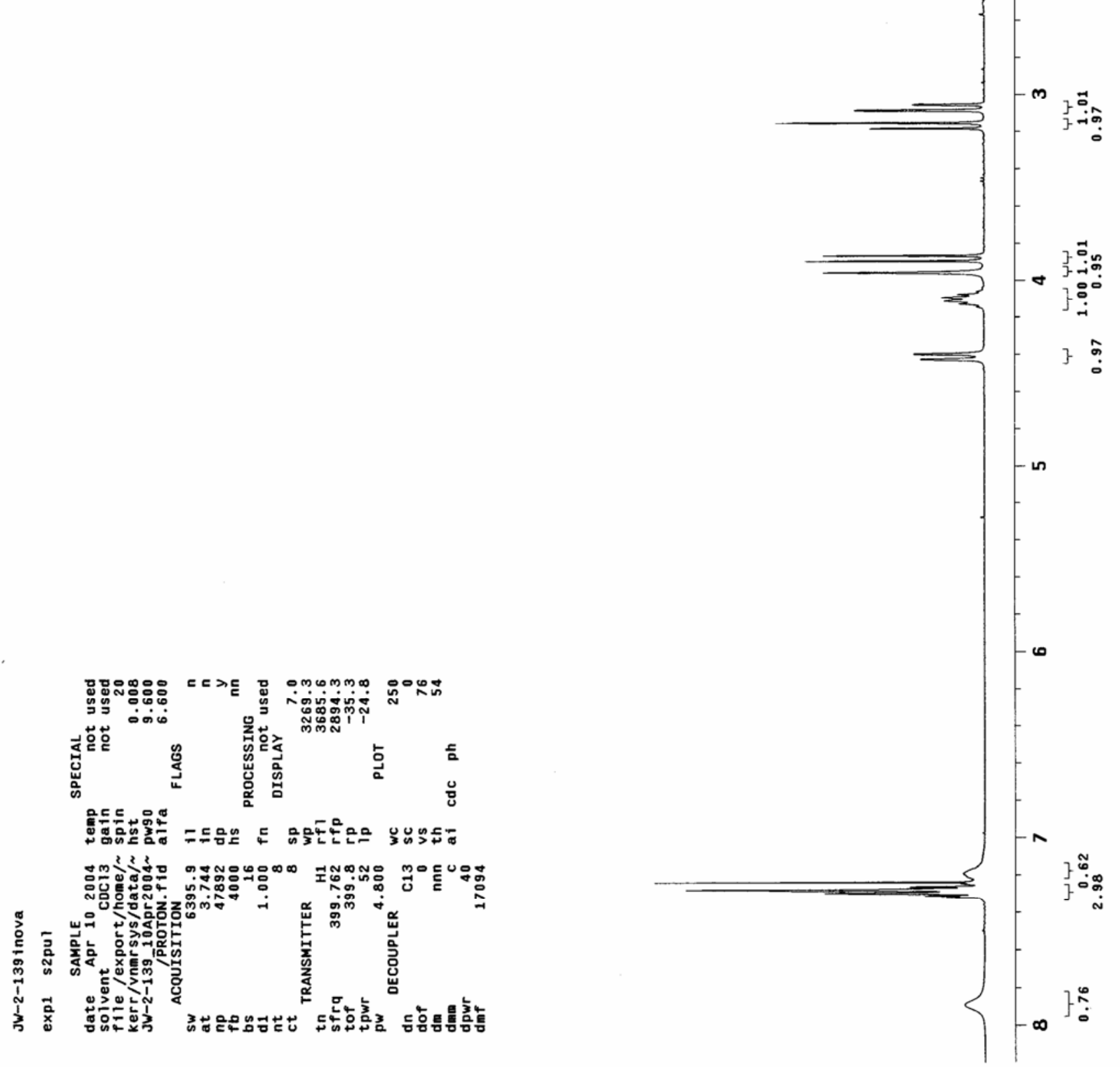

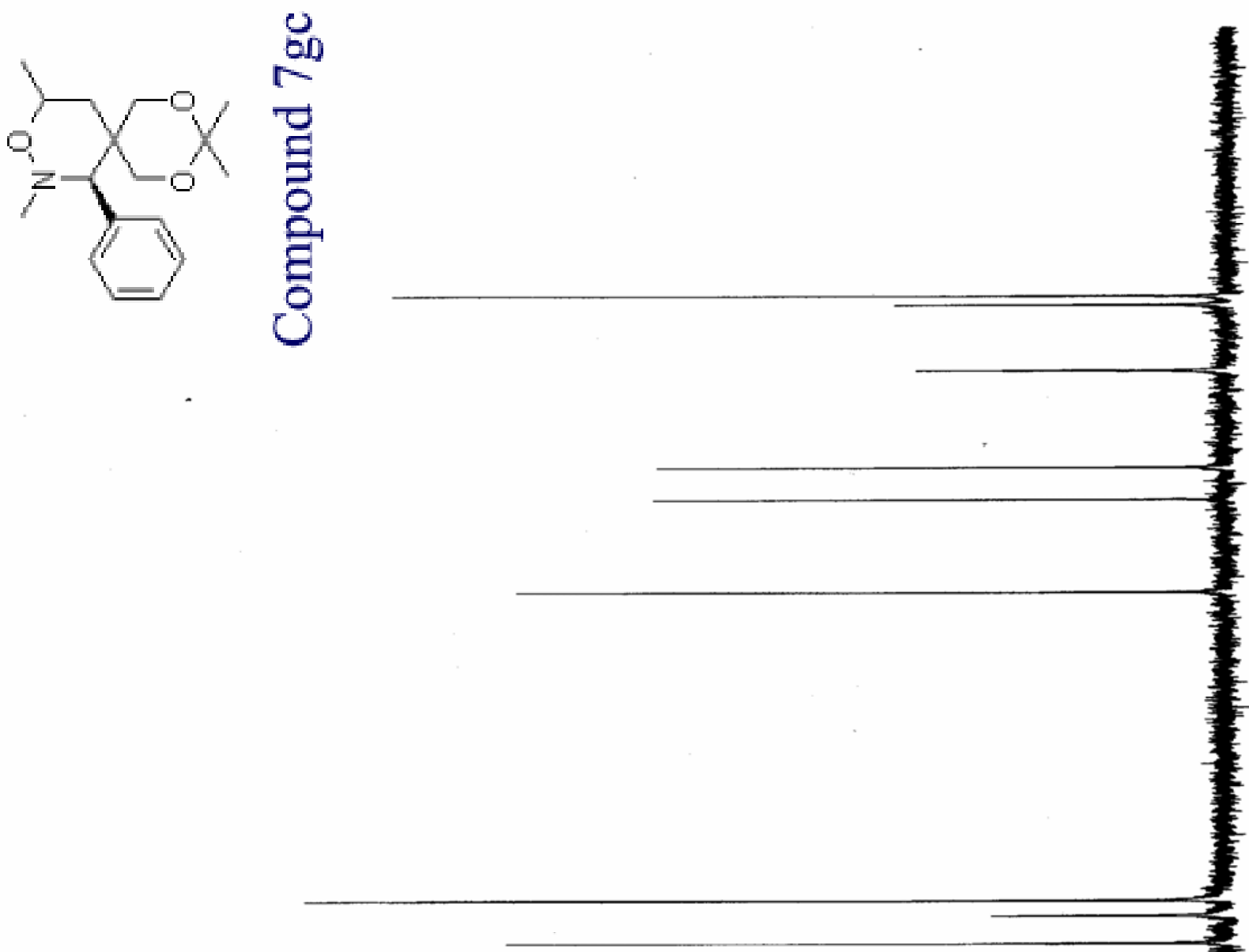

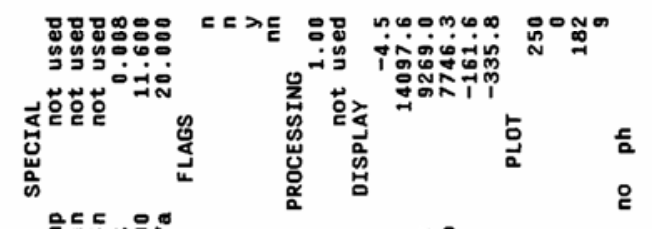

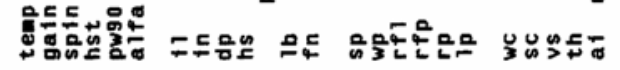

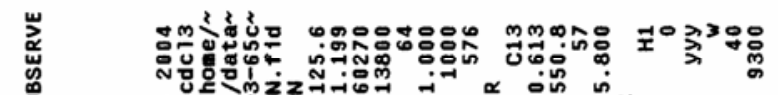

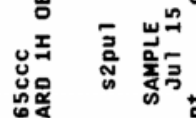

wis

I.

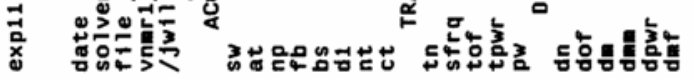

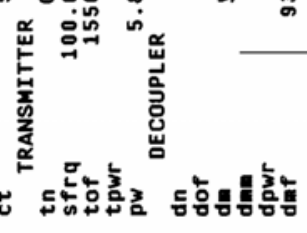


$=1$

㖣

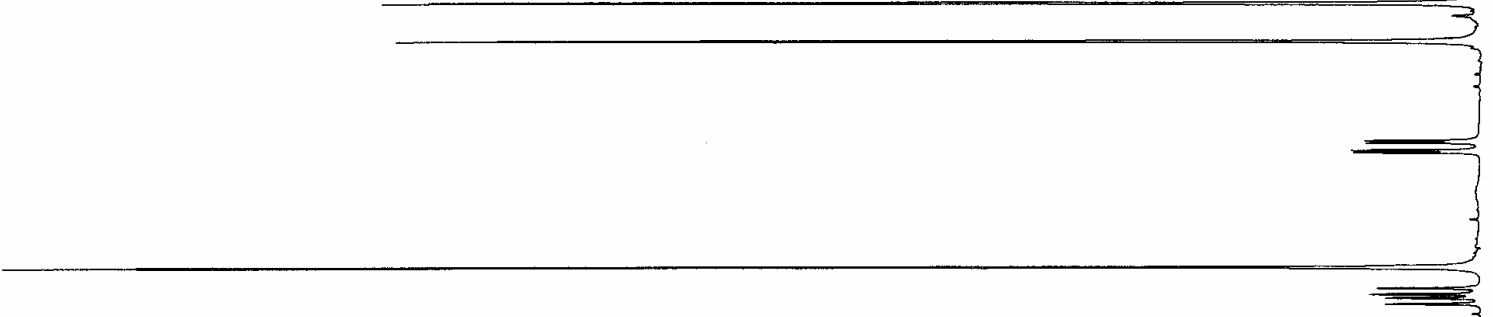

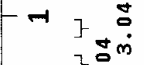

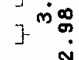

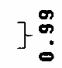

$N$

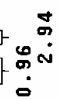

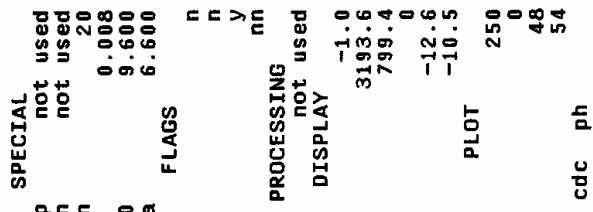

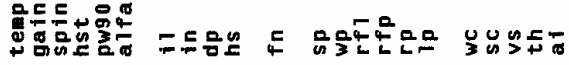

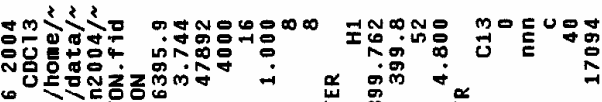

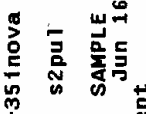

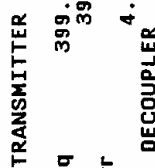

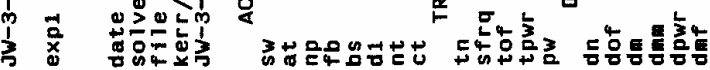

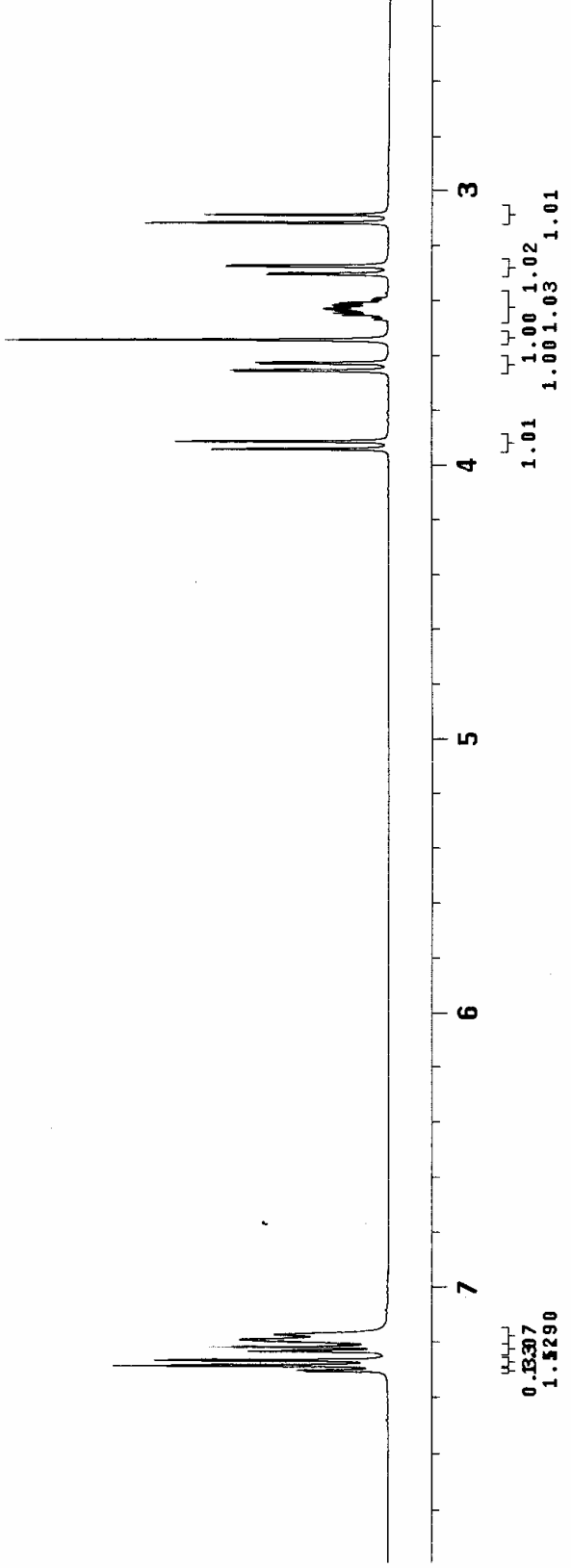



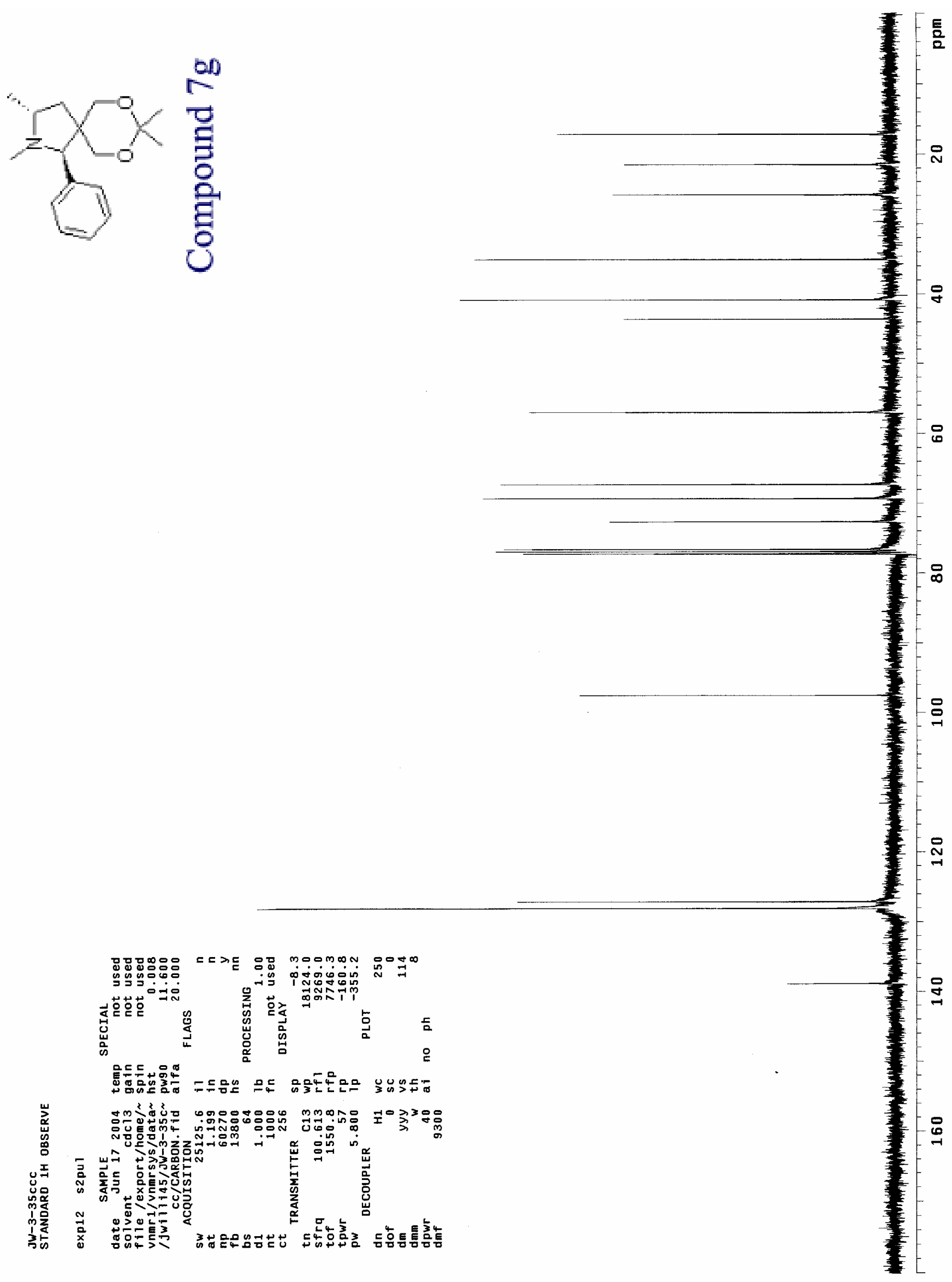


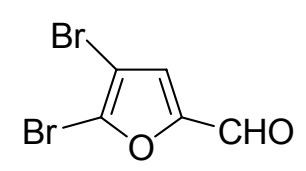

\section{Compound I}

Compound I was prepared using the procedure of Chiarello, J.; Joullie, M. M. Tetrahedron 1998, 44, 41-8, with changes made to the purification procedure. Freshly distilled furfural (20 $\mathrm{g}, 208 \mathrm{mmol})$ was added dropwise at $0{ }^{\circ} \mathrm{C}$ to aluminum chloride $(61 \mathrm{~g}, 456 \mathrm{mmol})$ under an argon atmosphere over a two hour period with mechanical stirring. Bromine (72.6 g, $454 \mathrm{mmol})$ was then added dropwise at $0{ }^{\circ} \mathrm{C}$ over a two hour period, after which stirring was discontinued and the reaction allowed to stand overnight. The reaction was quenched by carefully pouring the mixture into ice $(400 \mathrm{~mL})$ and then extracting the aqueous layer three times with ether. The combined organics were dried with $\mathrm{MgSO}_{4}$ and the solvent removed under reduced pressure. In the above reference the product was purified by distillation, but heating led to a black tar being produced in the distillation setup and no product being recovered. Purification using column chromatography on silica (hexanes/ethyl acetate as eluent) yielded I (24.85 g, 47\%) as an orange oil.<smiles>OCc1cc(Br)c(Br)o1</smiles>

\section{Compound II}

Dibromoaldehyde I (12.44 g, $48.96 \mathrm{mmol})$ was dissolved in methanol $(300 \mathrm{~mL})$ and cooled to 0 ${ }^{\circ} \mathrm{C}$. $\mathrm{NaBH}_{4}$ was added $(0.93 \mathrm{~g}, 24.48 \mathrm{mmol})$ in 0.1 gram portions over 15 minutes, after which the reaction was complete by TLC. $5 \mathrm{~mL}$ of water was added and the methanol removed under reduced pressure. The residual material was added to water $(200 \mathrm{~mL})$ and $5 \% \mathrm{HCl}(20 \mathrm{~mL})$ and the aqueous layer extracted 3 times with ethyl acetate. The combined organics were dried with $\mathrm{MgSO}_{4}$, and the solvent removed under reduced pressure. Dibromoalcohol II (12.40 g of red oil) was of sufficient purity to be used directly in the next step with out further purification. 


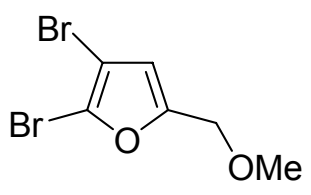

\section{Compound III}

THF (150 ml) was added to $\mathrm{NaH}$ (1.78 g, (2.96g of $60 \%$ dispersion in mineral oil) $73.9 \mathrm{mmol})$ that had been previously washed with hexanes under argon. The slurry was cooled to $0{ }^{\circ} \mathrm{C}$, and dibromoalcohol II (12.53 g, $49.3 \mathrm{mmol})$ in THF (75 mL) was added dropwise over 30 minutes, and then stirred 30 minutes at $0^{\circ} \mathrm{C}$. Methyl Iodide $(14.2 \mathrm{~g}, 98.6 \mathrm{mmol})$ in $\mathrm{THF}(75 \mathrm{~mL}) \mathrm{was}$ added dropwise over 30 minutes, and the reaction allowed to warm to room temperature and stirred overnight. After $13 \mathrm{hrs}$ TLC showed complete consumption of the starting material, and $10 \mathrm{~mL}$ of water was added to quench the excess $\mathrm{NaH}$. The THF was removed under reduced pressure and the residual material added to water $(200 \mathrm{~mL})$ and $5 \% \mathrm{HCl}(20 \mathrm{~mL})$. The aqueous portion was extracted 3 times with ethyl acetate and the combined organics dried with $\mathrm{MgSO}_{4}$ and the solvent removed under reduced pressure. Purification by column chromatography (hexanes/ethyl acetate as eluent) yielded dibromoether III (10.52 g, $39.2 \mathrm{mmol}, 80 \%$ for two steps) as a yellow oil.

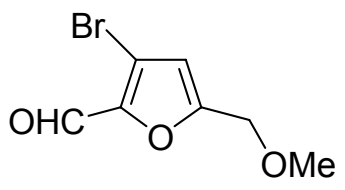

\section{Compound 16}

The procedure used is an adaptation from Zaluski, M-C., Robba, M., Bonhomme, M. Bull. Soc. Chim. Fr. 1970, 1838 for the preparation of a similar compound. n-BuLi $(22.91 \mathrm{~mL}$ of $1.6 \mathrm{M}$ in hexanes, $36.65 \mathrm{mmol})$ was added to anhydrous ether $(150 \mathrm{~mL})$ under an argon atmosphere and the solution was cooled to $-78{ }^{\circ} \mathrm{C}$. A solution of the dibromoether III $(9.00 \mathrm{~g}, 33.32 \mathrm{mmol})$ in ether $(100 \mathrm{~mL})$ was added dropwise to the $\mathrm{n}-\mathrm{BuLi}$, and after addition was complete the mixture was stirred for 30 minutes at $-78{ }^{\circ} \mathrm{C}$. DMF $(3.40 \mathrm{~g}, 46.65 \mathrm{mmol})$ in ether $(100 \mathrm{~mL})$ was then added dropwise, and after addition was complete the reaction was warmed to room temperature over two hours, and then refluxed for 30 minutes. The reaction was quenched by the addition of water $(5 \mathrm{~mL})$, and then $5 \% \mathrm{HCl}$ was added $(300 \mathrm{~mL})$. The layers were separated and the aqueous extracted 3 times with ether. The combined organics were washed with water and dried with $\mathrm{MgSO}_{4}$. Flash column chromatography on silica gel (hexanes/ethyl acetate as eluent) afforded 16 (3.78 g, $17.3 \mathrm{mmol}, 52 \%)$ as a pale yellow oil, which turned into a solid when placed in freezer. $\mathrm{MP}=$ low melting solid, $<40{ }^{\circ} \mathrm{C} ; \mathrm{R}_{\mathrm{f}}=0.37,20 \%$ ethyl acetate in hexanes; ${ }^{1} \mathrm{H}-\mathrm{NMR}$ $\left(400 \mathrm{MHz}, \mathrm{CDCl}_{3}\right): \delta=9.69(\mathrm{~s}, 1 \mathrm{H}), 6.60(\mathrm{t}, \mathrm{J}=0.8 \mathrm{~Hz}, 1 \mathrm{H}), 4.47(\mathrm{~d}, \mathrm{~J}=0.8 \mathrm{~Hz}, 2 \mathrm{H}), 3.43(\mathrm{~s}$, $3 \mathrm{H}) ;{ }^{13} \mathrm{C} \mathrm{NMR}\left(100 \mathrm{MHz}, \mathrm{CDCl}_{3}\right): \delta=176.0,158.4,147.6,114.6,113.3,66.5,58.9$; IR (thin film): $v_{\max }=3118,2933,2824,1676,1576,1521,1458,1374,1274,1193,1098,1031,967,906$, 807, 770; HRMS calc'd for $\mathrm{C}_{7} \mathrm{H}_{7} \mathrm{BrO}_{3}=217.9579$, found = 217.9585; 


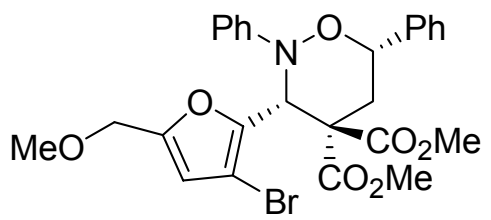

\section{Compound 18}

Phenylhydroxylamine (1.73 g, $15.82 \mathrm{mmol})$ and furfural 16 (3.61 g, $16.47 \mathrm{mmol})$ were dissolved in toluene $(100 \mathrm{~mL})$. Molecular sieves and $\mathrm{Yb}(\mathrm{OTf})_{3} \cdot \mathrm{xH}_{2} \mathrm{O}(0.817 \mathrm{~g}, 1.318 \mathrm{mmol})$ were added, and the reaction mixture stirred for $30 \mathrm{~min}$ at room temperature under argon. Cyclopropane 17 $(3.09 \mathrm{~g}, 13.18 \mathrm{mmol})$ was then added and the reaction mixture stirred for 18 hours. The reaction mixture was then filtered through celite to remove the sieves and the filter cake washed with ethyl acetate. The solvent was removed under reduced pressure, and methanol $(50 \mathrm{~mL})$ added to the crude material. The methanol slurry was stirred in an ice bath for 30 minutes, and cycloadduct 18 (4.88 g, $8.96 \mathrm{mmol}, 68 \%)$ was collected by filtration as a white powder. A crude NMR of the reaction mixture showed that what appeared to be the trans diastereomer was produced in approximately $8-12 \%$ yield, but it did not precipitate from methanol. M.P. $=149-$ $150{ }^{\circ} \mathrm{C} ; \mathrm{R}_{\mathrm{f}}=0.36,20 \%$ ethyl acetate in hexanes; ${ }^{1} \mathrm{H}-\mathrm{NMR}\left(600 \mathrm{MHz}, \mathrm{CDCl}_{3}\right): \delta=7.59(\mathrm{~d}, \mathrm{~J}=$ $8.4 \mathrm{~Hz}, 2 \mathrm{H}), 7.42(\mathrm{t}, \mathrm{J}=7.8 \mathrm{~Hz}, 2 \mathrm{H}), 7.36(\mathrm{tt}, \mathrm{J}=7.2,1.2 \mathrm{~Hz}, 1 \mathrm{H}), 7.20(\mathrm{t}, \mathrm{J}=7.2 \mathrm{~Hz}, 2 \mathrm{H}), 7.10$ $(\mathrm{d}, \mathrm{J}=8.4 \mathrm{~Hz}, 2 \mathrm{H}), 6.96(\mathrm{tt}, \mathrm{J}=7.2,1.2 \mathrm{~Hz}, 1 \mathrm{H}), 6.21(\mathrm{~s}, 1 \mathrm{H}), 5.81(\mathrm{~s}, 1 \mathrm{H}), 5.06(\mathrm{dd}, \mathrm{J}=12.2$, $2.7 \mathrm{~Hz}, 1 \mathrm{H}), 4.41(\mathrm{~d}, \mathrm{~J}=13.2 \mathrm{~Hz}, 1 \mathrm{H}), 4.37(\mathrm{~d}, \mathrm{~J}=13.2 \mathrm{~Hz}, 1 \mathrm{H}), 3.97(\mathrm{~s}, 3 \mathrm{H}), 3.60(\mathrm{~s}, 3 \mathrm{H}), 3.32$ $(\mathrm{s}, 3 \mathrm{H}), 3.01(\mathrm{dd}, \mathrm{J}=12.2,13.8 \mathrm{~Hz}, 1 \mathrm{H}), 2.76(\mathrm{ddd}, \mathrm{J}=13.8,2.7,0.6 \mathrm{~Hz}, 1 \mathrm{H}) ;{ }^{13} \mathrm{C}$ NMR $(100$ $\left.\mathrm{MHz}, \mathrm{CDCl}_{3}\right): \delta=169.2,167.8,152.9,148.2,146.9,140.0,128.5,128.4,128.3,126.9,123.1$, 117.2, 112.4, 102.9, 79.2, 66.2, 59.3, 58.2, 57.7, 53.6, 53.0, 33.6; IR (thin film): $v_{\max }=3062$, 3033, 2992, 2954, 2894, 2823, 1742, 1599, 1492, 1455, 1435, 1259, 1222, 1115, 1089, 1047, 987, 946, 807, 756, 698; HRMS calc'd for $\mathrm{C}_{26} \mathrm{H}_{26} \mathrm{BrNO}_{7}=543.0893$, found $=543.0895$

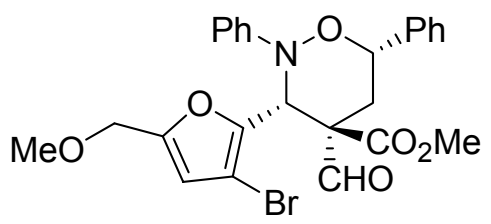

\section{Compound 19}

Cycloadduct 18 (1.00 g, $1.84 \mathrm{mmol})$ was dissolved in methylene chloride $(125 \mathrm{~mL})$ and then cooled to $-78{ }^{\circ} \mathrm{C}$ under argon. DIBALH $(6.84 \mathrm{~mL}$ of $1.5 \mathrm{M}$ solution in toluene, $10.26 \mathrm{mmol})$ was added dropwise and the reaction stirred for 15 minutes, after which multiply eluted TLC showed complete consumption of the starting material. Methanol $(10 \mathrm{~mL})$ was then added slowly to quench the excess DIBALH, and the mixture stirred at $-78{ }^{\circ} \mathrm{C}$ for 20 minutes, after which it was removed from the dry ice bath and allowed to warm to room temperature. The reaction was then poured into $5 \% \mathrm{HCl}(300 \mathrm{~mL})$ and the layers separated. The aqueous layer was extracted 3 times with methylene chloride, and the combined organics washed with $5 \% \mathrm{HCl}$. The organic layer was dried with $\mathrm{MgSO}_{4}$, and the solvent removed under reduced pressure. The residual material was purified by flash column chromatography on silica gel (hexanes/ethyl acetate as eluent) to yield aldehyde 19 (0.894 g, $1.74 \mathrm{mmol}, 95 \%)$ as a yellow waxy solid. $\mathrm{MP}=97-98{ }^{\circ} \mathrm{C} ; \mathrm{R}_{\mathrm{f}}=0.34$, 
$20 \%$ ethyl acetate in hexanes; ${ }^{1} \mathrm{H}-\mathrm{NMR}\left(600 \mathrm{MHz}, \mathrm{CDCl}_{3}\right): \delta=9.33(\mathrm{~s}, 1 \mathrm{H}), 7.60(\mathrm{dd}, \mathrm{J}=7.8$, $1.8 \mathrm{~Hz}, 2 \mathrm{H}), 7.42(\mathrm{tt}, \mathrm{J}=7.8,1.2 \mathrm{~Hz}, 2 \mathrm{H}), 7.37(\mathrm{tt}, \mathrm{J}=7.5,1.2 \mathrm{~Hz}, 1 \mathrm{H}), 7.22-7.20(\mathrm{~m}, 2 \mathrm{H}), 7.07$ $(\mathrm{dd}, \mathrm{J}=7.7,1.2 \mathrm{~Hz}, 2 \mathrm{H}), 7.00(\mathrm{tt}, \mathrm{J}=7.2,1.2 \mathrm{~Hz}, 1 \mathrm{H}), 6.24(\mathrm{~s}, 1 \mathrm{H}), 5.86(\mathrm{~s}, 1 \mathrm{H}), 5.22(\mathrm{dd}, \mathrm{J}=$ 11.7, $3.0 \mathrm{~Hz}, 1 \mathrm{H}), 4.39(\mathrm{~s}, 2 \mathrm{H}), 4.00(\mathrm{~s}, 3 \mathrm{H}), 3.32(\mathrm{~s}, 3 \mathrm{H}), 2.81(\mathrm{dd}, \mathrm{J}=14.4,11.7,1 \mathrm{H}), 2.65$ $(\mathrm{ddd}, \mathrm{J}=14.4,3.0,1.2 \mathrm{~Hz}, 1 \mathrm{H}) ;{ }^{13} \mathrm{C} \mathrm{NMR}\left(100 \mathrm{MHz}, \mathrm{CDCl}_{3}\right): \delta=194.4168 .6,153.6,147.8$, $146.0,140.0,128.53,128.47,128.3,126.8,123.5,117.6,112.4,103.6,79.1,66.1,63.6,58.5$, 57.8, 53.7, 30.7; IR (thin film): $v_{\max }=3063,3033,2953,2928,2909,2823,1750,1722,1598$, 1492, 1454, 1437, 1220, 1091,1073, 1048, 990, 966; HRMS calc'd for $\mathrm{C}_{25} \mathrm{H}_{24} \mathrm{BrNO}_{6}=$ 513.0787 , found $=513.0793$.

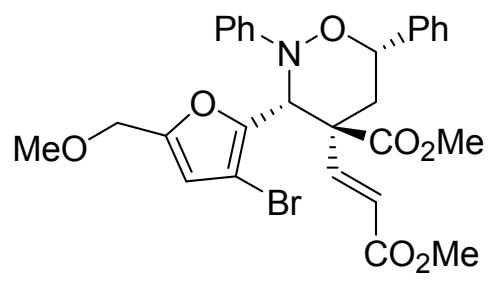

Compound 20

Trimethyl phosphonoacetate $(0.346 \mathrm{~g}, 1.898 \mathrm{mmol})$ was dissolved in THF $(30 \mathrm{~mL})$ under argon and cooled in an ice bath. $\mathrm{KO} t \mathrm{Bu}(0.213 \mathrm{~g}, 1.898 \mathrm{mmol})$ was added in small portions and after addition was complete, the slurry was stirred for 10 minutes. Aldehyde 19 (0.888 g, 1.725 $\mathrm{mmol})$ in THF $(30 \mathrm{~mL})$ was then added dropwise, and TLC indicated complete consumption of the starting material after 2 hours. Water $(5 \mathrm{~mL})$ was added, and the THF removed under reduced pressure. To the resulting residue was added water $(100 \mathrm{~mL}) 5 \% \mathrm{HCl}(20 \mathrm{~mL})$ and ethyl acetate $(50 \mathrm{~mL})$. The layers were separated, and the organic washed 3 times with ethyl acetate. The combined organics were dried with $\mathrm{MgSO}_{4}$, and the solvent removed under reduced pressure, and the residual material purified by column chromatography on silica gel (hexanes/ethyl acetate as eluent) to yield enoate $\mathbf{2 0}(0.720 \mathrm{~g}, 1.26 \mathrm{mmol}, 73 \%)$ as a sticky white foam. $\mathrm{R}_{\mathrm{f}}=0.36,25 \%$ ethyl acetate in hexanes; ${ }^{1} \mathrm{H}-\mathrm{NMR}\left(400 \mathrm{MHz}, \mathrm{CDCl}_{3}\right): \delta=7.60$ (app. d, J $=8.0 \mathrm{~Hz}, 2 \mathrm{H}), 7.44-7.34(\mathrm{~m}, 3 \mathrm{H}), 7.21-7.17(\mathrm{~m}, 2 \mathrm{H}), 7.05(\mathrm{dd}, \mathrm{J}=8.8,1.2 \mathrm{~Hz}, 2 \mathrm{H}), 6.96(\mathrm{tt}, \mathrm{J}=$ 7.2, 1.2 Hz, 1H), $6.70(\mathrm{~d}, \mathrm{~J}=16.0 \mathrm{~Hz}, 1 \mathrm{H}), 6.22(\mathrm{~s}, 1 \mathrm{H}), 5.88(\mathrm{~d}, \mathrm{~J}=16.0 \mathrm{~Hz}, 1 \mathrm{H}), 5.64(\mathrm{~s}, 1 \mathrm{H})$, $5.20(\mathrm{t}, \mathrm{J}=7.0 \mathrm{~Hz}, 1 \mathrm{H}), 4.39$ (app. s, 2H), $3.96(\mathrm{~s}, 3 \mathrm{H}), 3.67$ (s, 3H), $3.29(\mathrm{~s}, 3 \mathrm{H}), 2.67-2.65(\mathrm{~m}$, $2 \mathrm{H}) ;{ }^{13} \mathrm{C} \mathrm{NMR}\left(100 \mathrm{MHz}, \mathrm{CDCl}_{3}\right): \delta=171.7,165.9,152.8,148.2,146.8,145.0,140.3,128.5$, $128.2,126.8,123.2,122.8,117.4,112.5,103.6,79.5,66.2,61.2,57.653 .4,51.7,35.2$, an $\mathrm{sp}^{2}$ and $\mathrm{sp}^{3}$ carbon signal are missing presumably due to overlap. IR (thin film): $v_{\max }=3032,2952,2848$, 2822, 1731, 1653, 1599, 1492, 1454, 1436, 1319, 1280, 1219, 1090, 1056, 1031, 985, 758, 698; HRMS calc'd for $\mathrm{C}_{28} \mathrm{H}_{28} \mathrm{BrNO}_{7}=569.1049$, found $=569.1048$. 


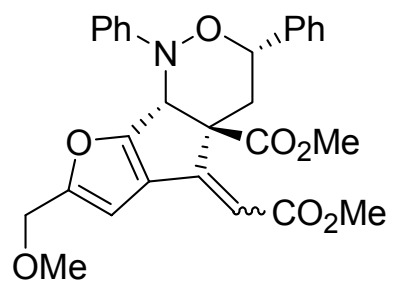

\section{Compound 21}

Enoate 20 (1.88 g, $3.30 \mathrm{mmol})$ was dissolved in DMF $(120 \mathrm{~mL})$ and $\mathrm{Ag}_{2} \mathrm{SO}_{4}(1.02 \mathrm{~g}, 3.30$ $\mathrm{mmol}), \mathrm{NEt}_{3}(1.67 \mathrm{~g}, 16.5 \mathrm{mmol})$, and $\mathrm{Pd}\left(\mathrm{PPh}_{3}\right)_{4}(0.953 \mathrm{~g}, 0.825 \mathrm{mmol})$ added. The mixture was heated at $120{ }^{\circ} \mathrm{C}$ under an argon atmosphere for 3 hours, after which more $\operatorname{Pd}\left(\mathrm{PPh}_{3}\right)_{4}(0.100 \mathrm{~g}$, $0.087 \mathrm{mmol}$ ) was added and the reaction heated for another 1.5 hours, after which TLC indicated that a small amount of starting material remained, but there was some decomposition apparent so the reaction was stopped. The reaction mixture was poured into water $(800 \mathrm{~mL})$ and $5 \% \mathrm{HCl}$ $(200 \mathrm{~mL})$ and extracted 3 times with ether. The combined organics were washed with water, and dried with $\mathrm{MgSO}_{4}$. The solvent was removed under reduced pressure and the residue purified by column chromatography on silica (hexanes/ethyl acetate as eluent) to produce recovered starting material $(0.337 \mathrm{~g}, 0.59 \mathrm{mmol})$ as an orange oil, and product $21(1.026 \mathrm{~g}, 2.095 \mathrm{mmol}, 64 \%, 78 \%$ BORSM) as an orange foam/oil. An NMR of the product showed a 5:1 mix of compounds which are assumed to be atrope isomers, or isomers around the enoate double bond. Upon cleavage of the $\mathrm{N}-\mathrm{O}$ bond in the next step the mixture of products converge into one, lending support for the product mixture being atrope isomers. The ratio is temperature dependant, and at lower temperature $\left(90^{\circ} \mathrm{C}\right)$ the ratio of isomers is $8: 1$, but the reaction proceeds very slowly. $\mathrm{R}_{\mathrm{f}}=$ $0.39,45 \%$ ethyl acetate in hexanes; ${ }^{1} \mathrm{H}-\mathrm{NMR}\left(400 \mathrm{MHz}, \mathrm{CDCl}_{3}\right)$ : major isomer $\delta=7.40-7.37$ $(\mathrm{m}, 2 \mathrm{H}), 7.35-7.28(\mathrm{~m}, 5 \mathrm{H}), 7.17(\mathrm{dd}, \mathrm{J}=8.8,0.8 \mathrm{~Hz}, 2 \mathrm{H}), 7.02(\mathrm{tt}, \mathrm{J}=7.2,0.8 \mathrm{~Hz}, 1 \mathrm{H}), 6.41(\mathrm{~s}$, $1 \mathrm{H}), 5.86(\mathrm{~s}, 1 \mathrm{H}), 5.45(\mathrm{dd}, \mathrm{J}=11.2,3.6 \mathrm{~Hz}, 1 \mathrm{H}), 5.41(\mathrm{~s}, 1 \mathrm{H}), 4.35(\mathrm{~d}, \mathrm{~J}=13.0 \mathrm{~Hz}, 1 \mathrm{H}), 4.30(\mathrm{~d}$, $\mathrm{J}=13.0 \mathrm{~Hz}, 1 \mathrm{H}), 3.77(\mathrm{~s}, 3 \mathrm{H}), 3.68(\mathrm{~s}, 3 \mathrm{H}), 3.36(\mathrm{~s}, 3 \mathrm{H}), 3.21(\mathrm{dd}, \mathrm{J}=14.4,3.6 \mathrm{~Hz}, 1 \mathrm{H}), 1.99$ $(\mathrm{dd}, \mathrm{J}=14.4,11.2 \mathrm{~Hz}, 1 \mathrm{H})$; representative peaks for minor isomer $\delta=6.34(\mathrm{~s}, 1 \mathrm{H}), 5.88(\mathrm{~s}, 1 \mathrm{H})$, 3.70 (s, 3H), 3.68 (s, 3H), 3.31 (s, 3H); IR (thin film): $v_{\max }=3059,3029,2950,1742,1735$, 1716, 1708, 1637, 1599, 1493, 1456, 1435, 1345, 1227, 1198, 1157, 1088, 915, 854, 753, 695; HRMS calc'd for $\mathrm{C}_{28} \mathrm{H}_{27} \mathrm{NO}_{7}=489.1788$, found $=489.1783$.<smiles>COCc1cc2c(o1)[C@@H](Nc1ccccc1)[C@](CC(O)c1ccccc1)(C(OC)OC)C2=CC(C)=O</smiles>

\section{Intermediate Aminoalcohol}

Compound $21(250 \mathrm{mg}, 0.512 \mathrm{mmol})$ was dissolved in methanol $(30 \mathrm{~mL})$ and $10 \%$ palladium on carbon $(125 \mathrm{mg})$ added. The reaction flask was evacuated and purged with hydrogen 3 times and then stirred under a hydrogen atmosphere for 2 hours, after which TLC showed complete consumption of the starting material. The reaction mixture was filtered through celite, and the 
filter pad washed with ethyl acetate. Removal of the solvent under reduced pressure led to the crude aminoalcohol as an orange oil. A small portion of this material was purified so that it could be characterized, but the aminoalcohol was of sufficient purity to be used directly in the next step. Characterization data for purified aminoalcohol. $\mathrm{R}_{\mathrm{f}}=0.30,50 \%$ ethyl acetate in hexanes; ${ }^{1} \mathrm{H}-\mathrm{NMR}\left(600 \mathrm{MHz}, \mathrm{CDCl}_{3}\right): \delta=7.30-7.26(\mathrm{~m}, 3 \mathrm{H}), 7.17-7.14(\mathrm{~m}, 4 \mathrm{H}), 6.75(\mathrm{t}, \mathrm{J}=$ $6.9 \mathrm{~Hz}, 1 \mathrm{H}), 6.48(\mathrm{~s}, 1 \mathrm{H}), 6.45(\mathrm{~d}, \mathrm{~J}=7.8 \mathrm{~Hz}, 2 \mathrm{H}), 6.03(\mathrm{~s}, 1 \mathrm{H}), 5.28(\mathrm{~s}, 1 \mathrm{H}), 4.70(\mathrm{dd}, \mathrm{J}=6.6$, 4.5, 1H), $4.40(\mathrm{~s}, 2 \mathrm{H}), 3.76(\mathrm{~s}, 3 \mathrm{H}), 3.72(\mathrm{~s}, 3 \mathrm{H}), 3.43(\mathrm{~s}, 3 \mathrm{H}), 2.97(\mathrm{dd}, \mathrm{J}=15.0,6.6 \mathrm{~Hz}, 1 \mathrm{H})$, $2.31(\mathrm{dd}, \mathrm{J}=15.0,4.5 \mathrm{~Hz}, 1 \mathrm{H}) ;{ }^{13} \mathrm{C}$ NMR $\left(150 \mathrm{MHz}, \mathrm{CDCl}_{3}\right): \delta=173.9,166.8,164.1,159.5$, $151.1,145.5,144.9,129.7,129.3,128.3,127.5,126.0,118.7,113.0,109.3,104.4,71.3,66.7$, $66.5,62.0,58.3,52.3,51.4,40.7$; IR (thin film): $v_{\max }=3600-3300$ broad, $3406,3059,3028$, 2950, 2823, 1719, 1635, 1603, 1514, 1499, 1436, 1374, 1346, 1255, 1197, 1150, 1092, 1014, 915, 859, 751, 693; HRMS calc'd for $\mathrm{C}_{28} \mathrm{H}_{29} \mathrm{NO}_{7}=491.1944$ found $=491.1948$.

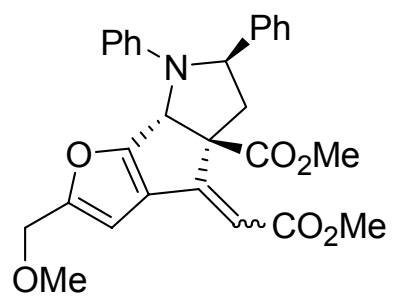

Compound 22

The crude aminoalcohol $(550 \mathrm{mg}, 1.123 \mathrm{mmol})$ was dissolved in methylene chloride $(50 \mathrm{~mL})$ and triethylamine $(341 \mathrm{mg}, 3.369 \mathrm{mmol})$ added and the reaction mixture stirred under argon. Mesyl chloride (154 mg, $1.347 \mathrm{mmol}$ ) was added dropwise, and after 30 minutes TLC indicated complete consumption of the starting material. Silica gel was added and the reaction mixture was pre-absorbed and purified by column chromatography on silica (hexanes/ethyl acetate as eluent) to yield pyrrolidine 22 (349 $\mathrm{mg}, 0.737 \mathrm{mmol}, 66 \%$ for 2 steps) as a white foamy solid. $\mathrm{R}_{\mathrm{f}}=0.50,40 \%$ ethyl acetate in hexanes; ${ }^{1} \mathrm{H}-\mathrm{NMR}\left(400 \mathrm{MHz}, \mathrm{CDCl}_{3}\right): \delta=7.29$ (app. d, J $=7.6$ $\mathrm{Hz}, 2 \mathrm{H}), 7.25(\mathrm{t}, \mathrm{J}=7.6 \mathrm{~Hz}, 2 \mathrm{H}), 7.20-7.16(\mathrm{~m}, 1 \mathrm{H}), 7.10-7.06(\mathrm{~m}, 2 \mathrm{H}), 6.77-6.73(\mathrm{~m}, 3 \mathrm{H}), 6.35$ $(\mathrm{s}, 1 \mathrm{H}), 5.91(\mathrm{~s}, 1 \mathrm{H}), 5.59(\mathrm{~s}, 1 \mathrm{H}), 4.44(\mathrm{dd}, \mathrm{J}=9.6,6.4 \mathrm{~Hz}, 1 \mathrm{H}), 4.25(\mathrm{~d}, \mathrm{~J}=13.2,1 \mathrm{H}), 4.14(\mathrm{~d}, \mathrm{~J}$ $=13.2 \mathrm{~Hz}, 1 \mathrm{H}), 3.72(\mathrm{~s}, 3 \mathrm{H}), 3.67(\mathrm{~s}, 3 \mathrm{H}), 3.22(\mathrm{dd}, \mathrm{J}=13.8,9.6 \mathrm{~Hz}, 1 \mathrm{H}), 3.18(\mathrm{~s}, 3 \mathrm{H}), 2.59(\mathrm{dd}$, $\mathrm{J}=13.8,6.4 \mathrm{~Hz}, 1 \mathrm{H}) ;{ }^{13} \mathrm{C}$ NMR $\left(150 \mathrm{MHz}, \mathrm{CDCl}_{3}\right): \delta=172.3,166.5,165.1,160.1,153.7$, $145.3,141.7,131.4,128.5,128.4,127.0,126.4,119.7,118.5,107.0,103.3,69.2,66.7,66.3,62.2$, 58.0, 52.6, 51.1, 45.5; IR (thin film): $v_{\max }=3029,2949,1741,1735,1716,1637,1600,1498$, $1457,1436,1345,1232,1176,1087,915,756,732$; HRMS calc'd for $\mathrm{C}_{28} \mathrm{H}_{27} \mathrm{NO}_{6}=473.1838$, found $=473.1848$; 


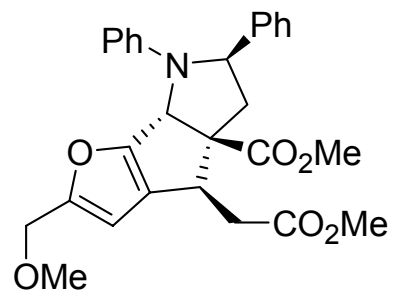

\section{Compound 23}

Compound $22(150 \mathrm{mg}, 0.317 \mathrm{mmol})$ was dissolved in methanol $(40 \mathrm{~mL})$ and $\mathrm{NiCl}_{2} \cdot 6 \mathrm{H}_{2} \mathrm{O}(23$ $\mathrm{mg}, 0.095 \mathrm{mmol}$ ) added. The reaction mixture was cooled to $-40{ }^{\circ} \mathrm{C}$, and sodium borohydride $(600 \mathrm{mg}, 15.85 \mathrm{mmol}$ ) was added in $50 \mathrm{mg}$ portions every 3 minutes, and then stirred for 30 minutes at $-40{ }^{\circ} \mathrm{C}$. The reaction was filtered through celite while still cold, and the filter cake washed with ethyl acetate. The organic layers were poured into water $(400 \mathrm{~mL})$, and the layers separated. The aqueous layer was washed three times with ethyl acetate, and the combined organics washed once with water. The organics were dried with $\mathrm{MgSO}_{4}$, and the solvent removed under reduced pressure. The residual material was purified by column chromatography on silica (hexanes/ethyl acetate as eluent) to yield 23 (100 mg, $0.209 \mathrm{mmol}, 66 \%)$ as a white foam, that could be crystallized from slow diffusion of methylene chloride into hexanes to yield colorless cubes. $\mathrm{MP}=144-145{ }^{\circ} \mathrm{C} ; \mathrm{R}_{\mathrm{f}}=0.50,40 \%$ ethyl acetate in hexanes; ${ }^{1} \mathrm{H}-\mathrm{NMR}(600$ $\left.\mathrm{MHz}, \mathrm{CDCl}_{3}\right): \delta=7.22(\mathrm{t}, \mathrm{J}=7.5 \mathrm{~Hz}, 2 \mathrm{H}), 7.19-7.14(\mathrm{~m}, 3 \mathrm{H}), 7.04(\mathrm{~d}, \mathrm{~J}=7.5 \mathrm{~Hz}, 2 \mathrm{H}), 6.73-$ $6.68(\mathrm{~m}, 3 \mathrm{H}), 6.16(\mathrm{~s}, 1 \mathrm{H}), 5.88(\mathrm{~s}, 1 \mathrm{H}), 4.94(\mathrm{~d}, \mathrm{~J}=7.8 \mathrm{~Hz}, 1 \mathrm{H}), 4.32(\mathrm{~d}, \mathrm{~J}=12.6 \mathrm{~Hz}, 1 \mathrm{H}), 4.27$ $(\mathrm{d}, \mathrm{J}=12.6 \mathrm{~Hz}, 1 \mathrm{H}), 3.67$ (s, 3H), $3.38(\mathrm{dd}, \mathrm{J}=10.2,4.5 \mathrm{~Hz}, 1 \mathrm{H}) ; 3.36(\mathrm{~s}, 3 \mathrm{H}), 3.27(\mathrm{~s}, 3 \mathrm{H}), 2.94$ $(\mathrm{d}, \mathrm{J}=12.6 \mathrm{~Hz}, 1 \mathrm{H}), 2.69(\mathrm{dd}, \mathrm{J}=12.6,7.8 \mathrm{~Hz}, 1 \mathrm{H}), 2.43(\mathrm{dd}, \mathrm{J}=16.2,4.5 \mathrm{~Hz}, 1 \mathrm{H}), 2.29(\mathrm{dd}, \mathrm{J}$ $=16.2,10.2 \mathrm{~Hz}, 1 \mathrm{H}) ;{ }^{13} \mathrm{C}$ NMR $\left(100 \mathrm{MHz}, \mathrm{CDCl}_{3}\right): \delta=172.5,172.0,158.1,156.7,144.8$, 141.3, 129.0, 128.2, 127.2, 126.7, 126.1, 116.5, 114.0, 108.0, 66.9, 66.7, 63.1, 61.6, 58.1, 51.9, 51.7, 45.0, 41.2, 37.0; IR (thin film): $v_{\max }=2951,1734,1599,1504,1437,1362,1224,1175$, 1084, 933, 911, 750, 692; HRMS calc'd for $\mathrm{C}_{28} \mathrm{H}_{29} \mathrm{NO}_{6}=475.1995$, found $=475.2000$; Relative stereochemistry confirmed by X-ray crystallography.

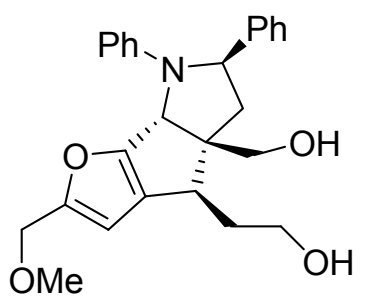

Compound 24

Compound 23 (115 mg, $0.24 \mathrm{mmol})$ was dissolved in THF $(10 \mathrm{~mL})$ and cooled to $0{ }^{\circ} \mathrm{C}$ under an argon atmosphere. $\mathrm{LiAlH}_{4}(0.725 \mathrm{~mL}$ of a $1 \mathrm{M}$ solution in ether, $0.725 \mathrm{mmol})$ was added dropwise and after 15 minutes TLC indicated complete consumption of the starting material. Water ( 8 drops) was added cautiously and the reaction stirred for 10 minutes. The resulting precipitate was removed by filtration through celite and the filter cake washed with ethyl acetate. The organics were then dried with $\mathrm{MgSO}_{4}$, and the solvent removed under reduced pressure. 
The residual material was purified by flash column chromatography on silica (hexanes/ethyl acetate as eluent) to yield diol $\mathbf{2 4}(70 \mathrm{mg}, 0.168 \mathrm{mmol}, 70 \%)$ as a yellow foam/solid. $\mathrm{R}_{\mathrm{f}}=0.35$, $100 \%$ ethyl acetate; ${ }^{1} \mathrm{H}-\mathrm{NMR}\left(600 \mathrm{MHz}, \mathrm{CDCl}_{3}\right): \delta=7.25(\mathrm{t}, \mathrm{J}=7.8,2 \mathrm{H}), 7.19(\mathrm{~d}, \mathrm{~J}=7.8 \mathrm{~Hz}$, 2H), 7.17-7.13 (m, 3H), 6.71-6.68 (m, 3H), $6.20(\mathrm{~s}, 1 \mathrm{H}), 5.15(\mathrm{~s}, 1 \mathrm{H}), 4.81(\mathrm{dd}, \mathrm{J}=8.4,4.2 \mathrm{~Hz}$, $1 \mathrm{H}), 4.28(\mathrm{~d}, \mathrm{~J}=12.6,1 \mathrm{H}), 4.20(\mathrm{~d}, \mathrm{~J}=12.6 \mathrm{~Hz}, 1 \mathrm{H}), 3.84-3.80(\mathrm{~m}, 2 \mathrm{H}), 3.73-3.69(\mathrm{~m}, 1 \mathrm{H}), 3.65$ (d, J = 11.4 Hz, 1H), 3.31 (s, 3H), $2.90(\mathrm{dd}, \mathrm{J}=10.4,3.9 \mathrm{~Hz}, 1 \mathrm{H}), 2.49-2.43(\mathrm{~m}, 2 \mathrm{H}), 2.18-2.13$ (m, $1 \mathrm{H}), 2.08-1.88$ (br. s, $2 \mathrm{H}), 1.58-1.53(\mathrm{~m}, 1 \mathrm{H}) ;{ }^{13} \mathrm{C} \mathrm{NMR}\left(150 \mathrm{MHz}, \mathrm{CDCl}_{3}\right): \delta=158.0$, 156.0, 145.6, 144.0, 129.8, 128.6, 128.5, 126.4, 126.1, 116.8, 114.6, 108.1, 66.9, 63.3, 63.0, 62.8, 61.8, 61.2, 58.0, 44.0, 41.2, 34.4; IR (thin film): $v_{\max }=3405$ (broad), 3060, 3027, 2932, 2879, $1599,1503,1450,1355,1267,1185,1079,1037,936,749,702$; HRMS calc'd for $\mathrm{C}_{26} \mathrm{H}_{29} \mathrm{NO}_{4}=$ 419.2097, found $=419.2098$.

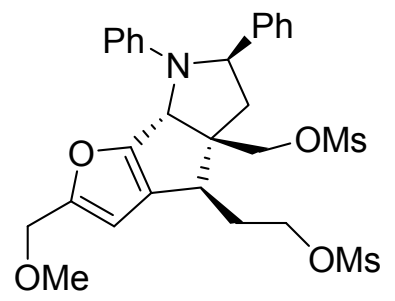

Compound 25

Diol 24 (70 mg, $0.167 \mathrm{mmol})$, triethylamine $(96 \mathrm{mg}, 0.948 \mathrm{mmol})$ and DMAP (10 mg) were added to methylene chloride $(10 \mathrm{~mL})$ and cooled to $-78{ }^{\circ} \mathrm{C}$ under argon. Mesyl Chloride $(72 \mathrm{mg}$, $0.631 \mathrm{mmol}$ ) was added and the reaction stirred for $3 \mathrm{hrs}$ at $-78{ }^{\circ} \mathrm{C}$. TLC showed that the diol had been consumed so the reaction was warmed to room temperature, and directly absorbed onto silica gel. Purification by column chromatography (hexanes/ethyl acetate as eluent) yielded bismesylate 25 (90 $\mathrm{mg}, 0.159 \mathrm{mmol}, 95 \%)$ as a white fluffy solid that could be crystallized from methylene chloride/hexanes to colorless needles for melting point. MP = turns orange and decomposes at $150^{\circ} \mathrm{C} ; \mathrm{R}_{\mathrm{f}}=0.63,75 \%$ ethyl acetate in hexanes; ${ }^{1} \mathrm{H}-\mathrm{NMR}\left(600 \mathrm{MHz}, \mathrm{CDCl}_{3}\right)$ : $\delta$ $=7.31(\mathrm{t}, 7.8 \mathrm{~Hz}, 2 \mathrm{H}), 7.22-7.19(\mathrm{~m}, 5 \mathrm{H}), 6.76(\mathrm{t}, \mathrm{J}=7.2 \mathrm{~Hz}, 1 \mathrm{H}), 6.71(\mathrm{~d}, \mathrm{~J}=8.4 \mathrm{~Hz}, 2 \mathrm{H}), 6.35$ (s, 1H), $5.22(\mathrm{~s}, 1 \mathrm{H}), 4.92(\mathrm{~d}, \mathrm{~J}=7.8 \mathrm{~Hz}, 1 \mathrm{H}), 4.39(\mathrm{~d}, \mathrm{~J}=10.5 \mathrm{~Hz}, 1 \mathrm{H}), 4.39-4.35(\mathrm{~m}, 2 \mathrm{H}), 4.30$ $(\mathrm{d}, \mathrm{J}=12.6 \mathrm{~Hz}, 1 \mathrm{H}), 4.24(\mathrm{~d}, \mathrm{~J}=12.6 \mathrm{~Hz}, 1 \mathrm{H}), 4.14(\mathrm{~d}, \mathrm{~J}=10.5 \mathrm{~Hz}, 1 \mathrm{H}), 3.35(\mathrm{~s}, 3 \mathrm{H}), 3.05(\mathrm{~s}$, $3 \mathrm{H}), 2.97(\mathrm{dd}, \mathrm{J}=11.4,3.6 \mathrm{~Hz}, 1 \mathrm{H}), 2.63(\mathrm{dd}, \mathrm{J}=13.2,9.6 \mathrm{~Hz}, 1 \mathrm{H}), 2.55(\mathrm{~d}, \mathrm{~J}=13.2 \mathrm{~Hz}, 1 \mathrm{H})$, 2.42-2.36 (m, 1H), 2.31 (br. s, 3H), 1.66-1.60 (m, 1H); $\left.{ }^{13} \mathrm{C} \mathrm{NMR} \mathrm{(100} \mathrm{MHz,} \mathrm{CDCl}_{3}\right): \delta=157.5$, $156.9,144.9,143.4,129.0,128.8,127.8,127.0,126.2,117.3,114.1,108.2,69.7,67.4,66.8,62.8$, 61.1, 60.5, 58.2, 43.6, 40.4, 37.5, 35.5, 30.9; IR (thin film): 3027, 2936, 1760, 1599, 1504, 1450, $1355,1174,1090,957,912,841,731,694$, 669; HRMS calc'd for $\mathrm{C}_{28} \mathrm{H}_{33} \mathrm{NO}_{8} \mathrm{~S}_{2}=575.1648$, found $=575.1640$. 


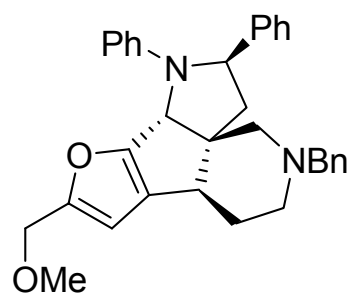

\section{Compound 26}

Bis-mesylate 25 (65 $\mathrm{mg}, 0.113 \mathrm{mmol})$ was added to absolute ethanol $(12 \mathrm{~mL})$ and THF $(\sim 1.5$ $\mathrm{mL}$ ) was added to solubilize 25 . $\mathrm{BnNH}_{2}(348 \mathrm{mg}, 3.25 \mathrm{mmol})$ was added and the reaction mixture refluxed under argon for 14 hours. The solvent was removed under reduced pressure, and the residue redissolved in methylene chloride, and preabsorbed on silica gel. Column chromatography on silica (hexanes/ethyl acetate as eluent) yielded piperidine 26 (52 $\mathrm{mg}, 0.106$ mmol, $95 \%)$ as a white foam. $\mathrm{R}_{\mathrm{f}}=0.51,40 \%$ ethyl acetate in hexanes; ${ }^{1} \mathrm{H}-\mathrm{NMR}(600 \mathrm{MHz}$, $\left.\mathrm{CDCl}_{3}\right): \delta=7.28-7.27(\mathrm{~m}, 2 \mathrm{H}), 7.24-7.21(\mathrm{~m}, 3 \mathrm{H}), 7.15-7.11(\mathrm{~m}, 5 \mathrm{H}), 6.98-6.96(\mathrm{~m}, 2 \mathrm{H}), 6.69-$ $6.66(\mathrm{~m}, 3 \mathrm{H}), 6.17(\mathrm{~s}, 1 \mathrm{H}), 5.33(\mathrm{~s}, 1 \mathrm{H}), 4.71(\mathrm{dd}, \mathrm{J}=9.6,2.4 \mathrm{~Hz}, 1 \mathrm{H}), 4.30(\mathrm{~d}, \mathrm{~J}=12.6 \mathrm{~Hz}, 1 \mathrm{H})$, 4.23 (d, J = 12.6 Hz, 1H), 3.49 (d, J = 13.2 Hz, 1H), 3.34 (s, 3H), 3.29 (d, J = 13.2 Hz, 1H), 2.97 (d, $12.6 \mathrm{~Hz}, 1 \mathrm{H}), 2.71(\mathrm{dd}, \mathrm{J}=13.2,9.6 \mathrm{~Hz}, 1 \mathrm{H}), 2.69-2.66(\mathrm{~m}, 1 \mathrm{H}), 2.56(\mathrm{dd}, \mathrm{J}=9.9,6.9 \mathrm{~Hz}$, $1 \mathrm{H}), 2.10-2.02(\mathrm{~m}, 2 \mathrm{H}), 1.89(\mathrm{dd}, \mathrm{J}=12.6,3.0 \mathrm{~Hz}, 1 \mathrm{H}), 1.77(\mathrm{~d}, \mathrm{~J}=12.6,1 \mathrm{H}), 1.54-1.48(\mathrm{~m}$, $1 \mathrm{H}) ;{ }^{13} \mathrm{C}$ NMR $\left(150 \mathrm{MHz}, \mathrm{CDCl}_{3}\right): \delta=158.9,155.6,146.1,144.2,138.6,131.7,128.7,128.6$, $128.3,128.2,126.9,126.2,125.9,116.4,114.4,107.0,67.0,64.2,62.8,62.1,60.8,58.5,57.9$, 52.0, 47.4, 38.4, 32.4; IR (thin film): $v_{\max }=3061,3027,2933,2805,2760,1598,1503,1450$, 1357, 1266, 1182, 1089, 1027, 993, 930, 908, 865, 819, 747, 699; HRMS calc'd for $\mathrm{C}_{33} \mathrm{H}_{34} \mathrm{~N}_{2} \mathrm{O}_{2}$ $=490.2620$, found $=490.2617$. 


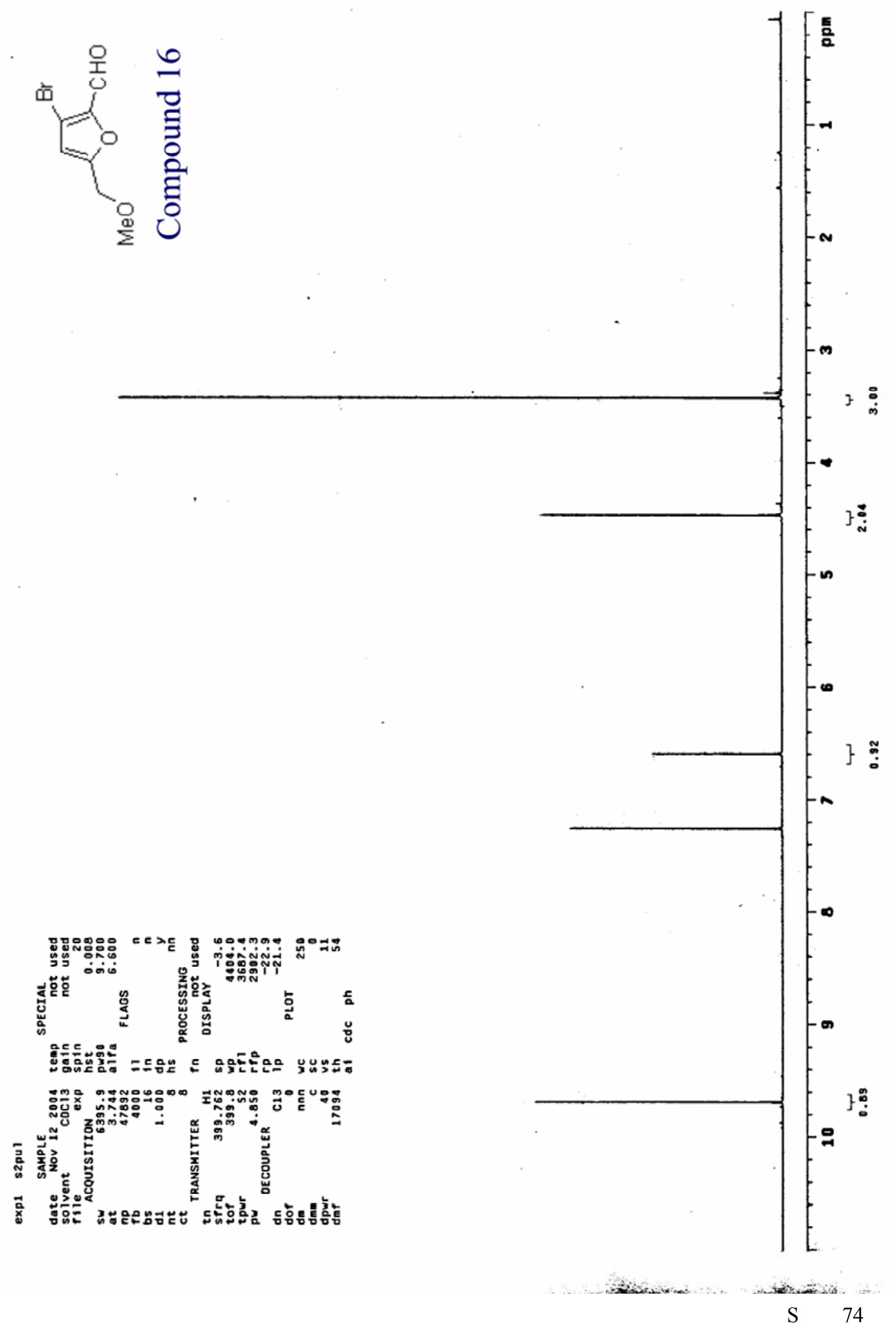



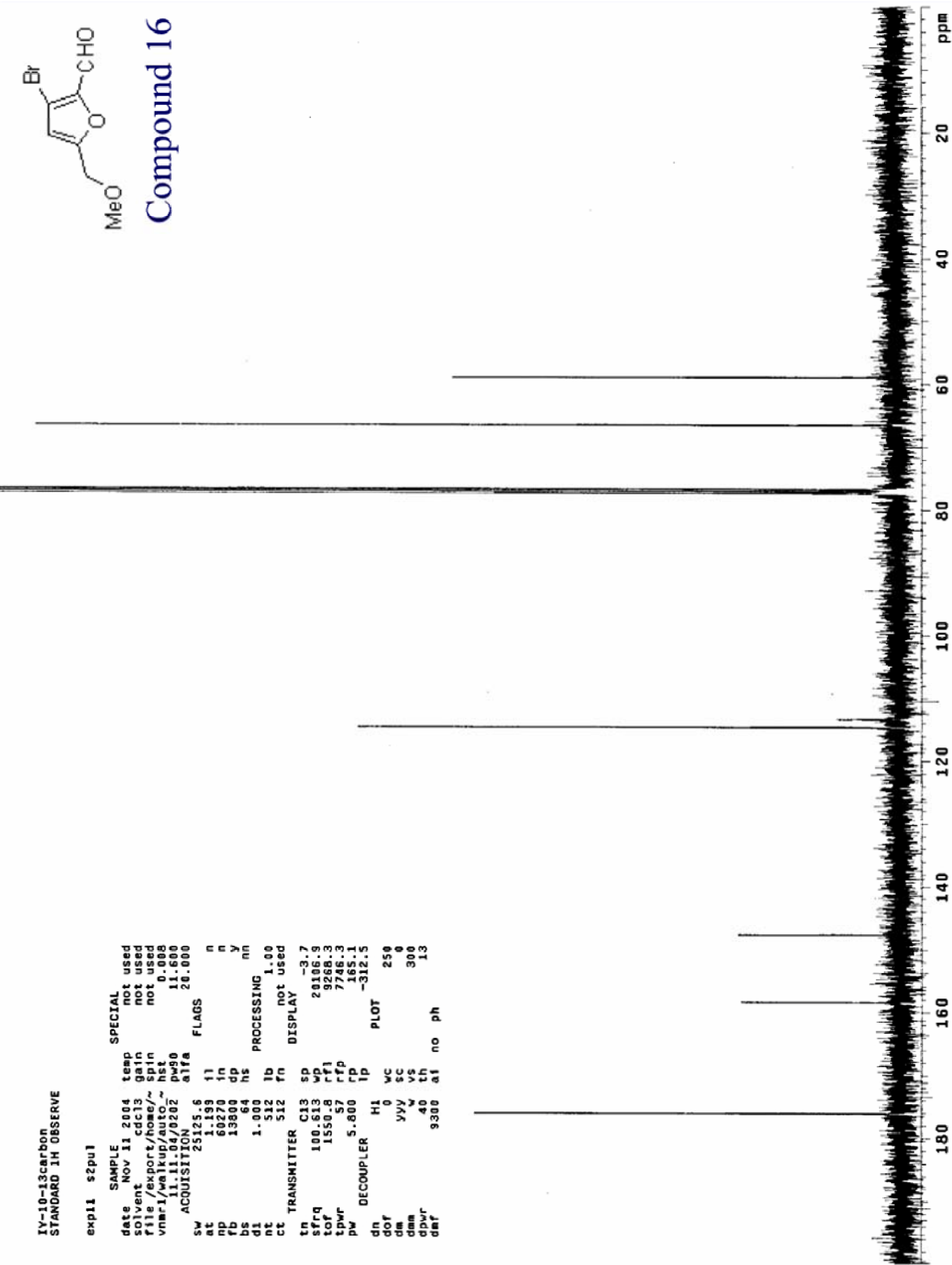

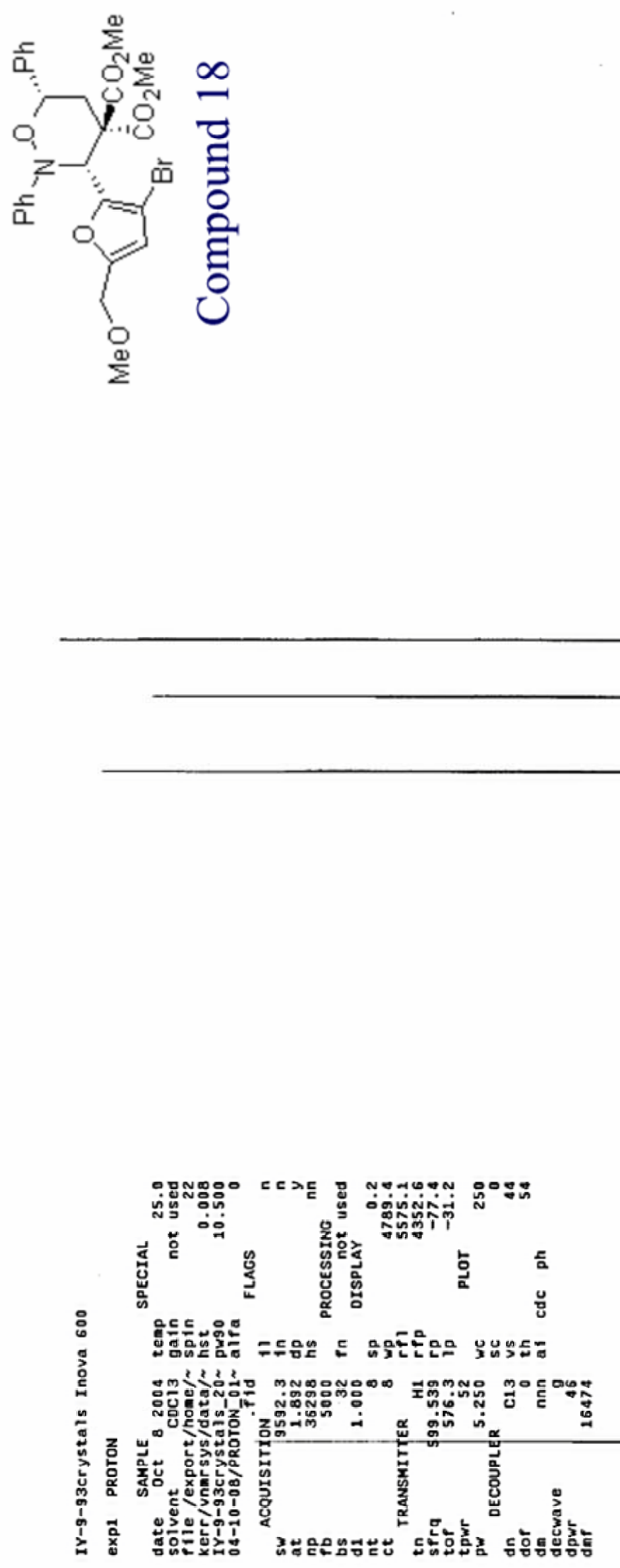
(2)

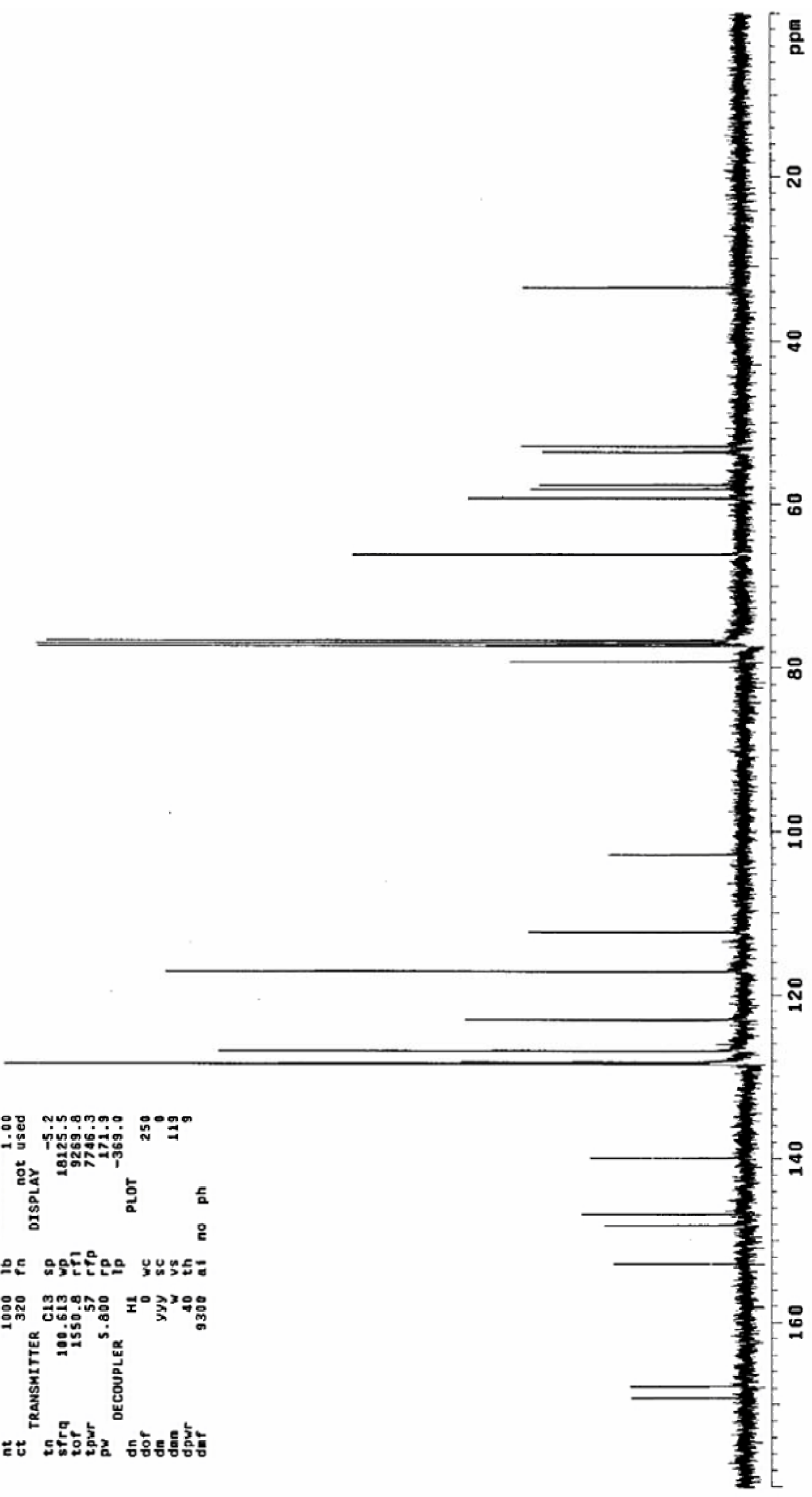




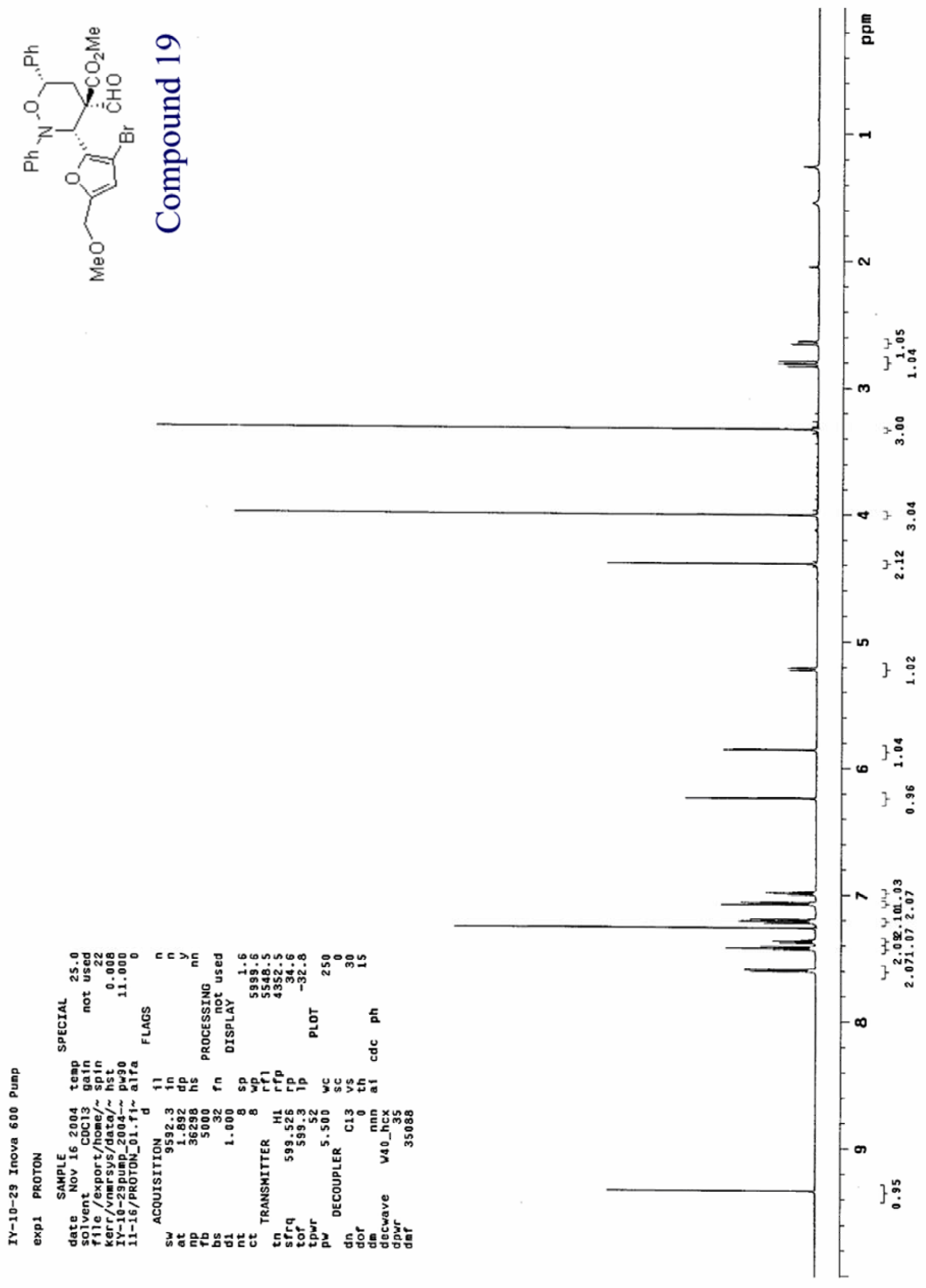



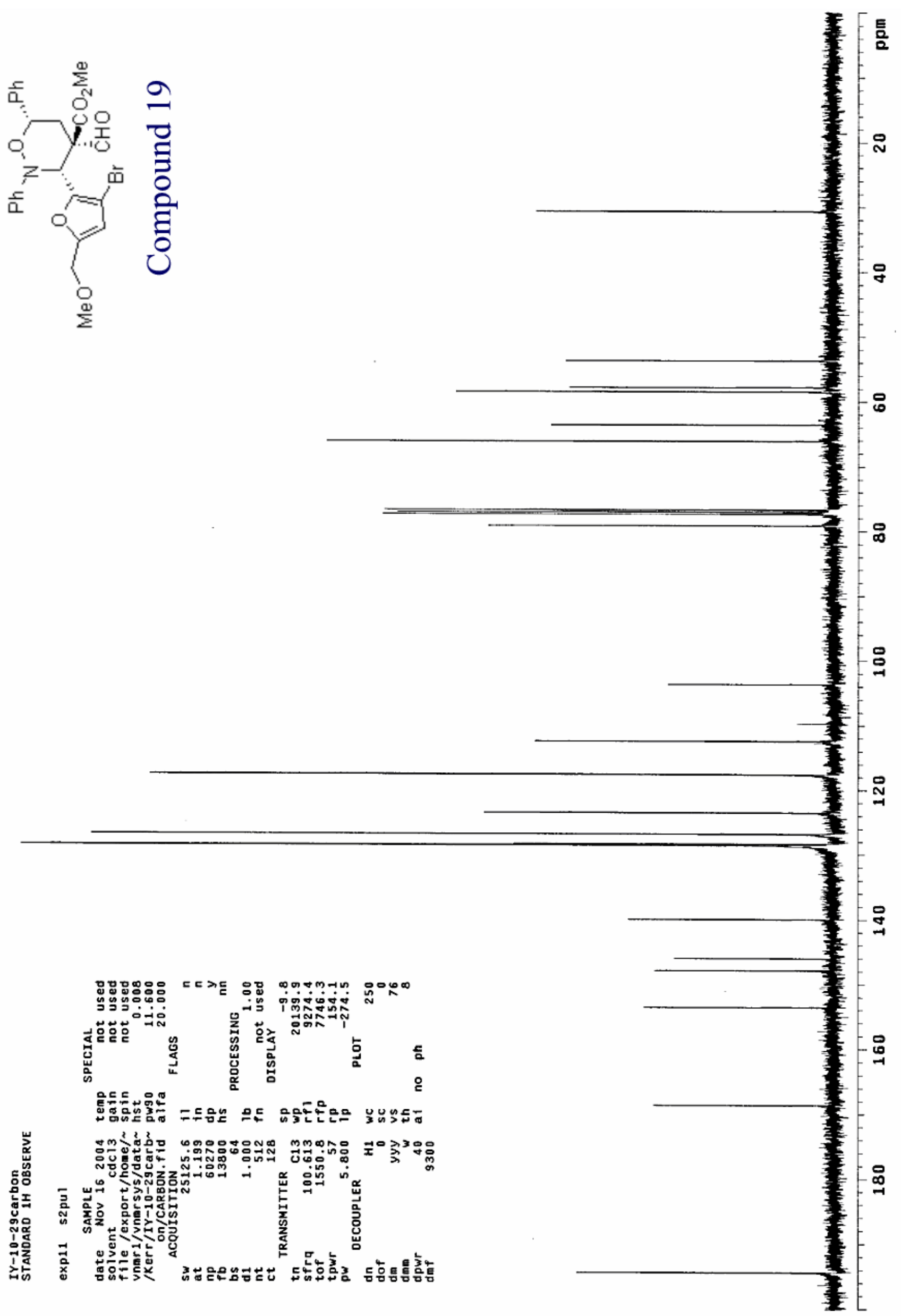

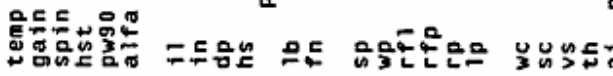

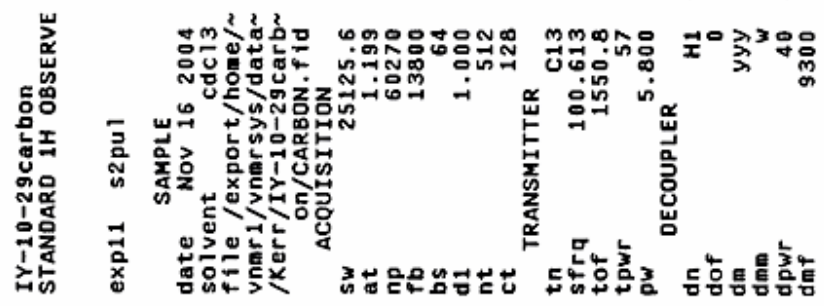



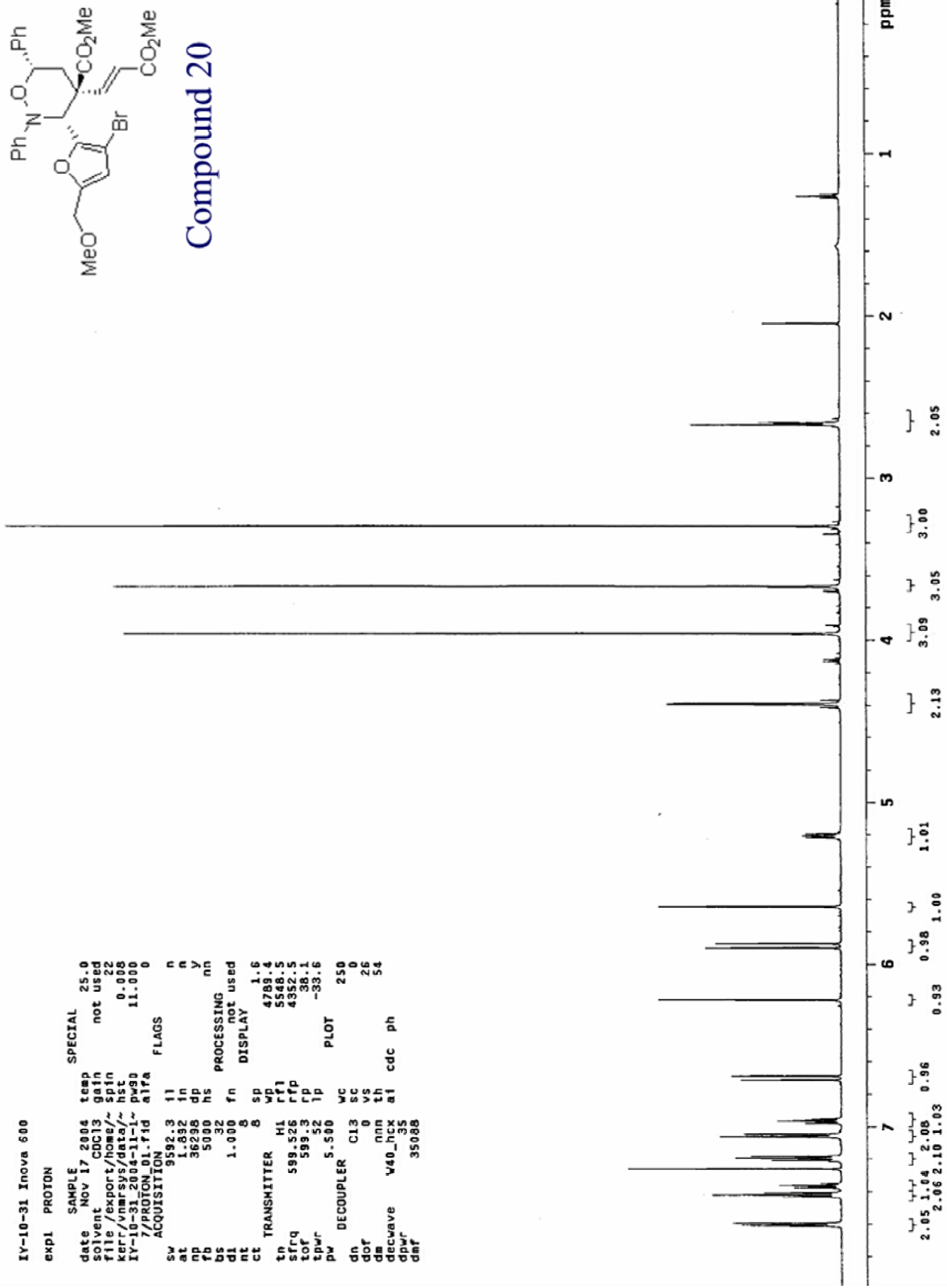

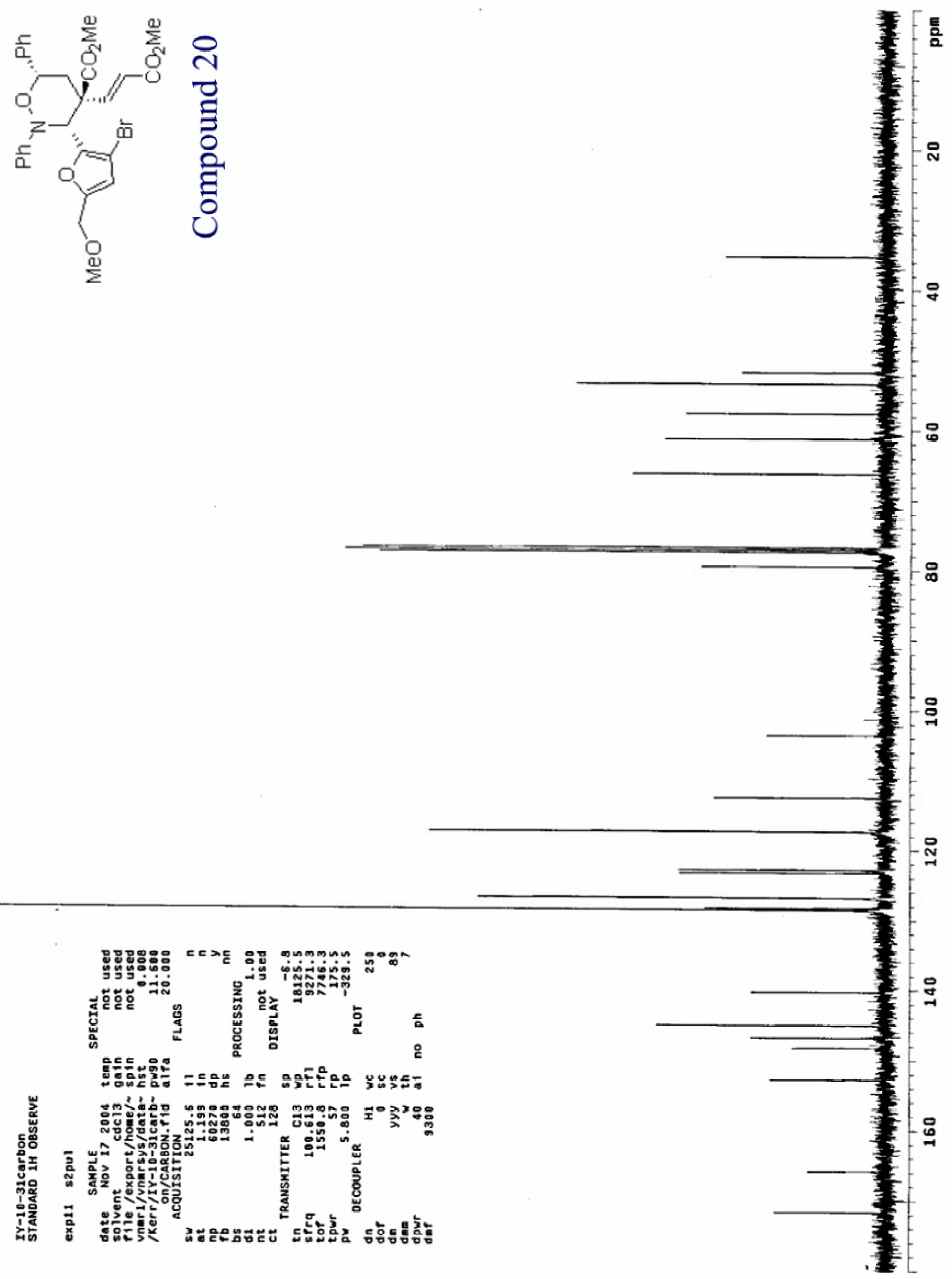

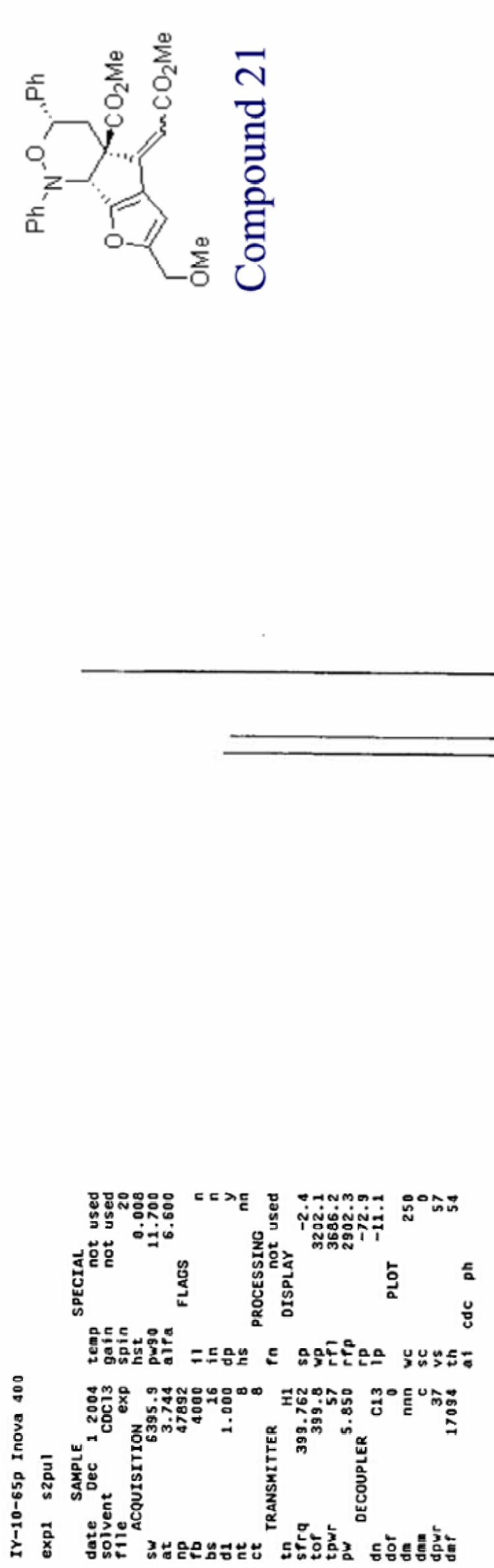

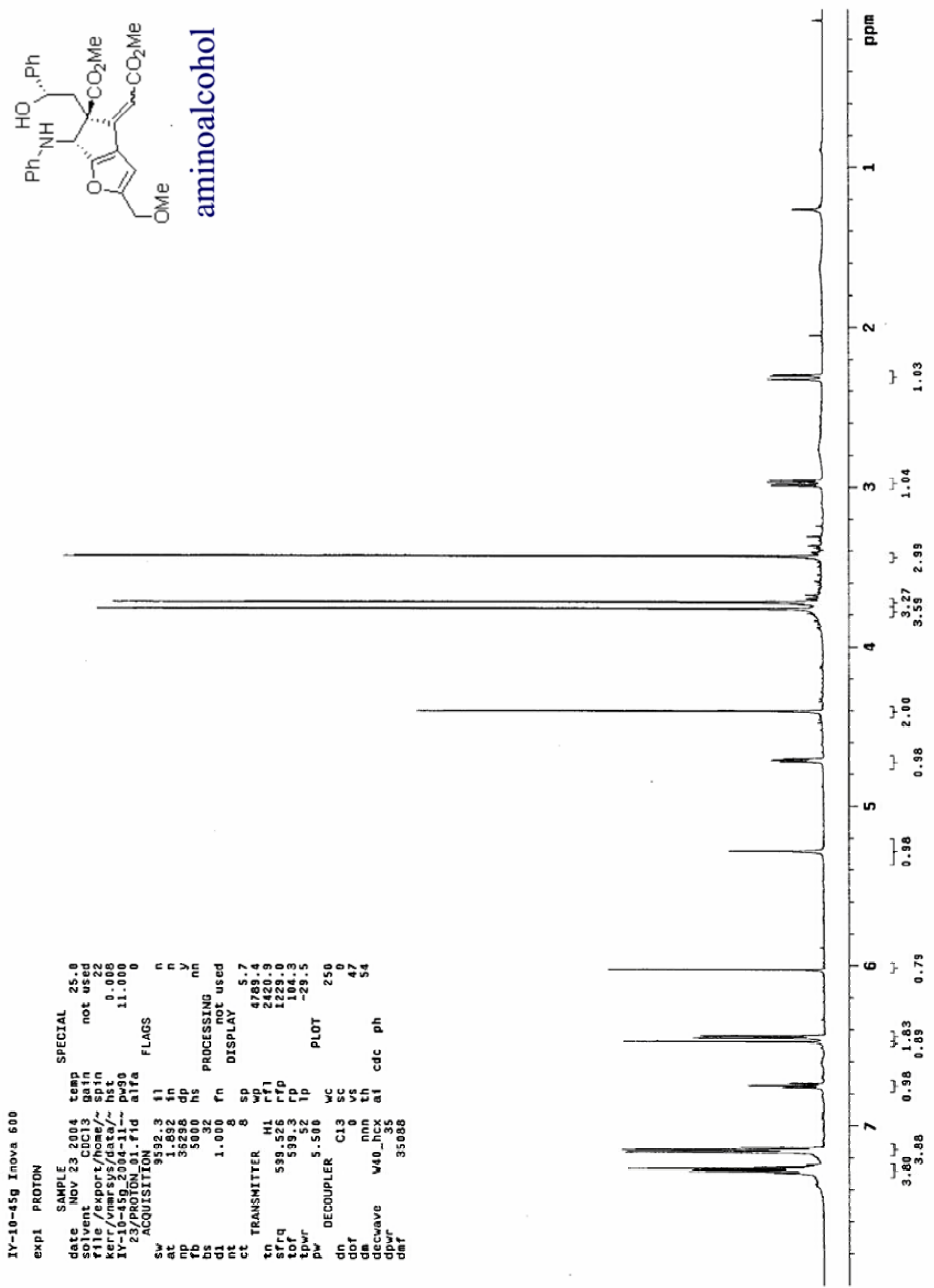
(1)

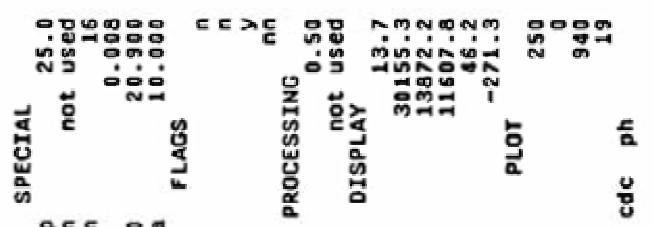

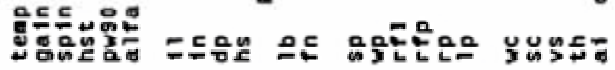

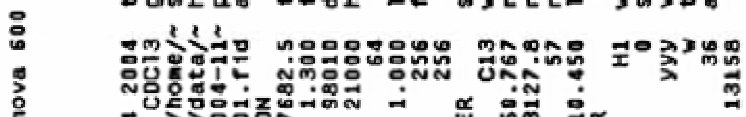

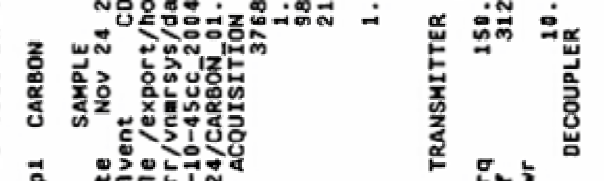

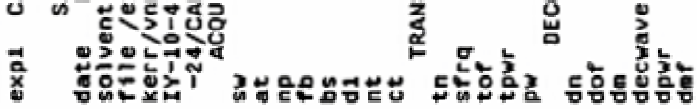

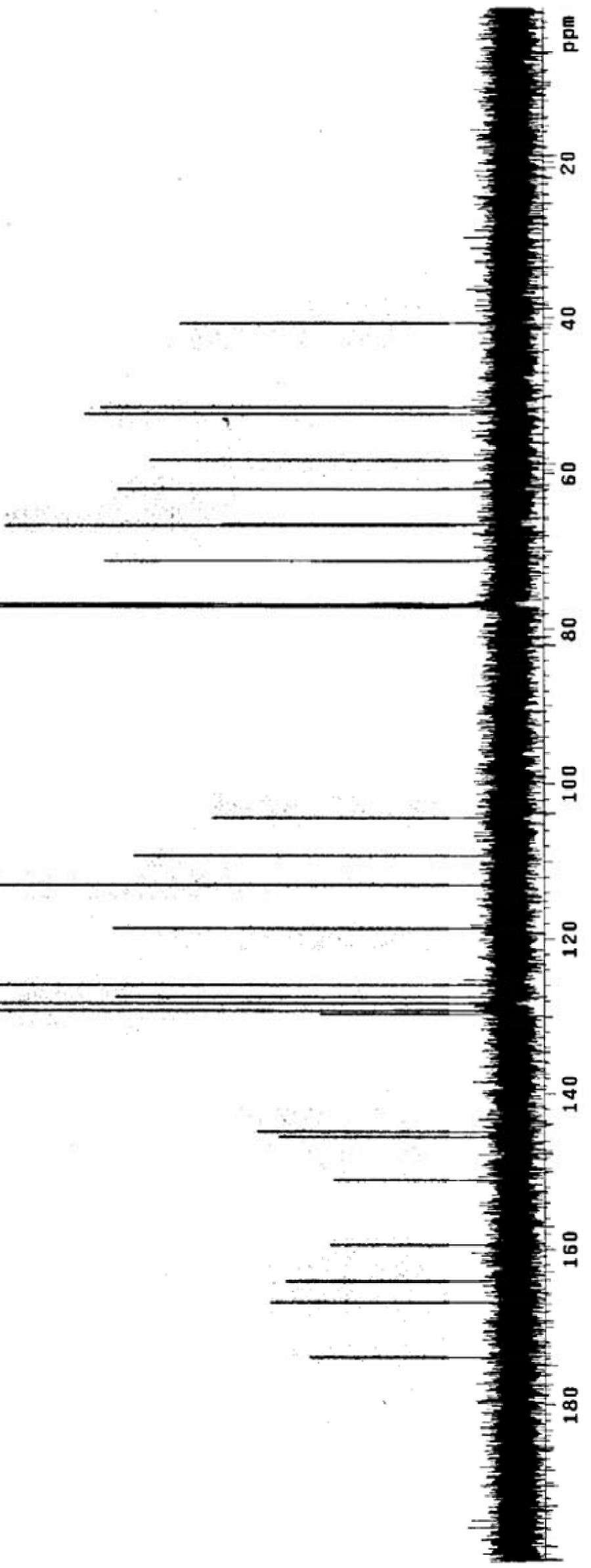



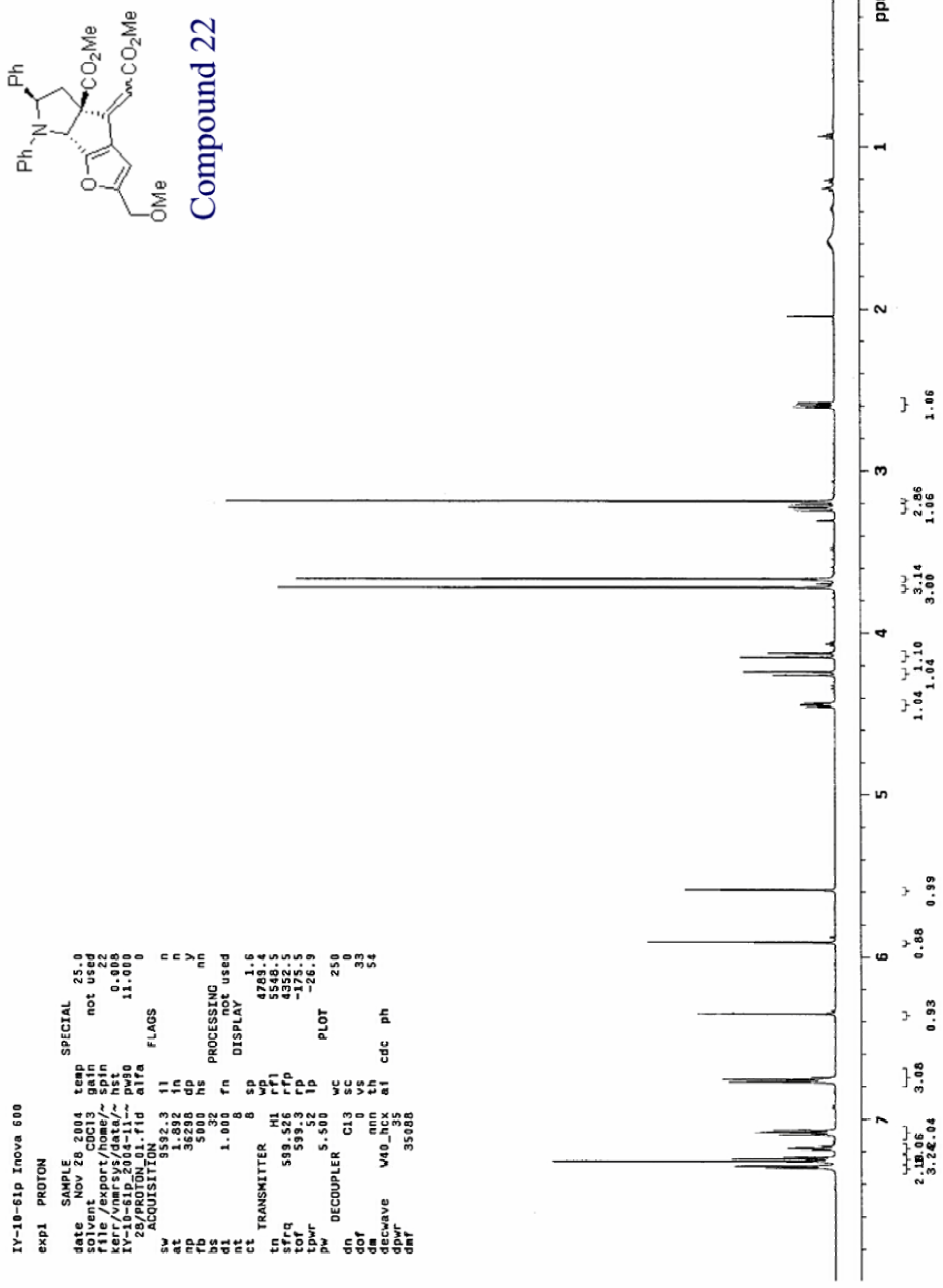

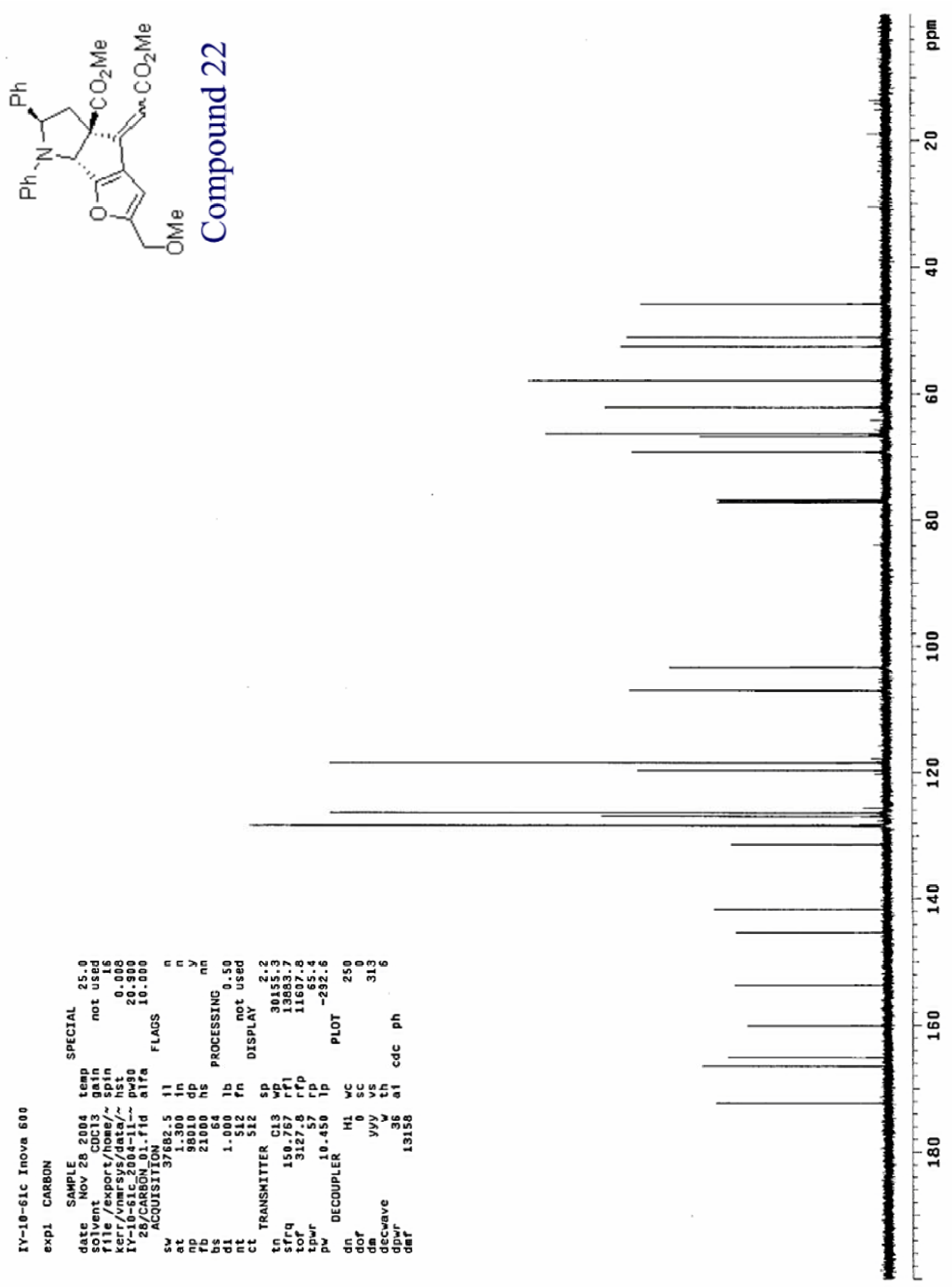

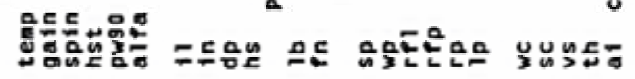

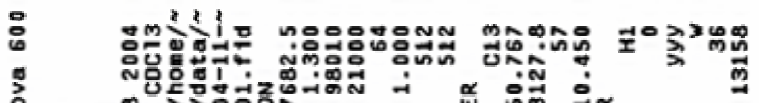

z w w

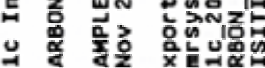

至

i

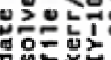

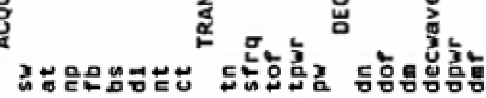



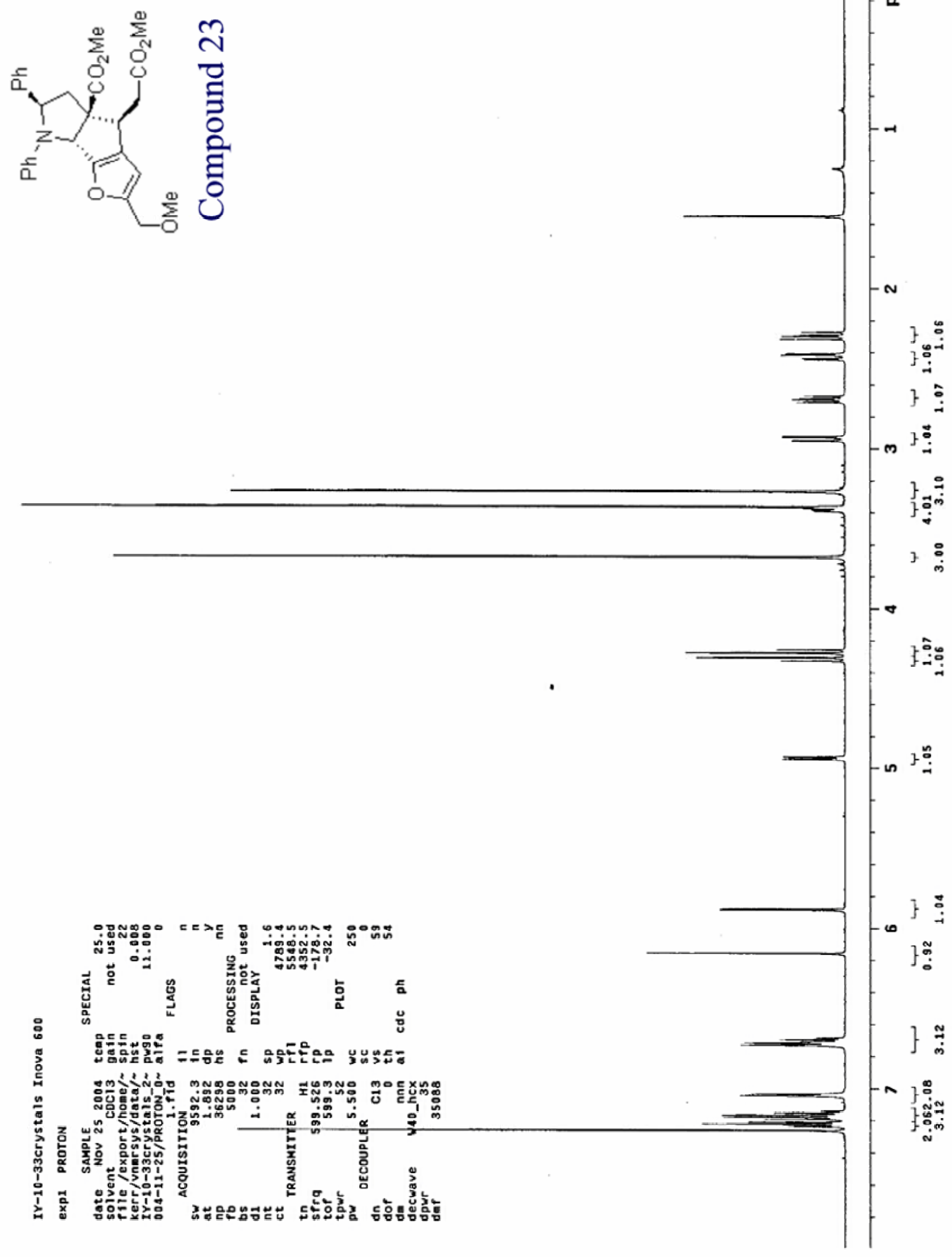
(1)

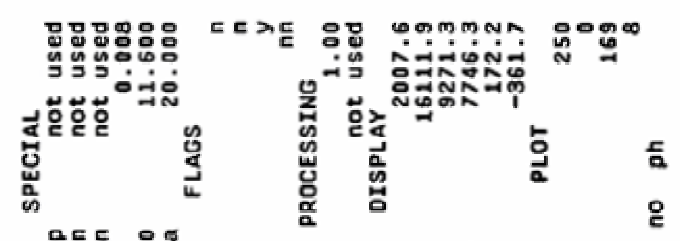

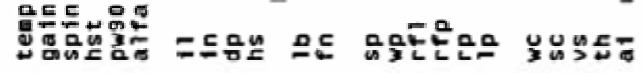

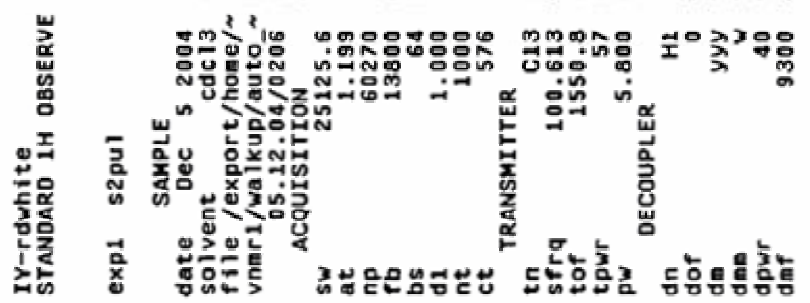



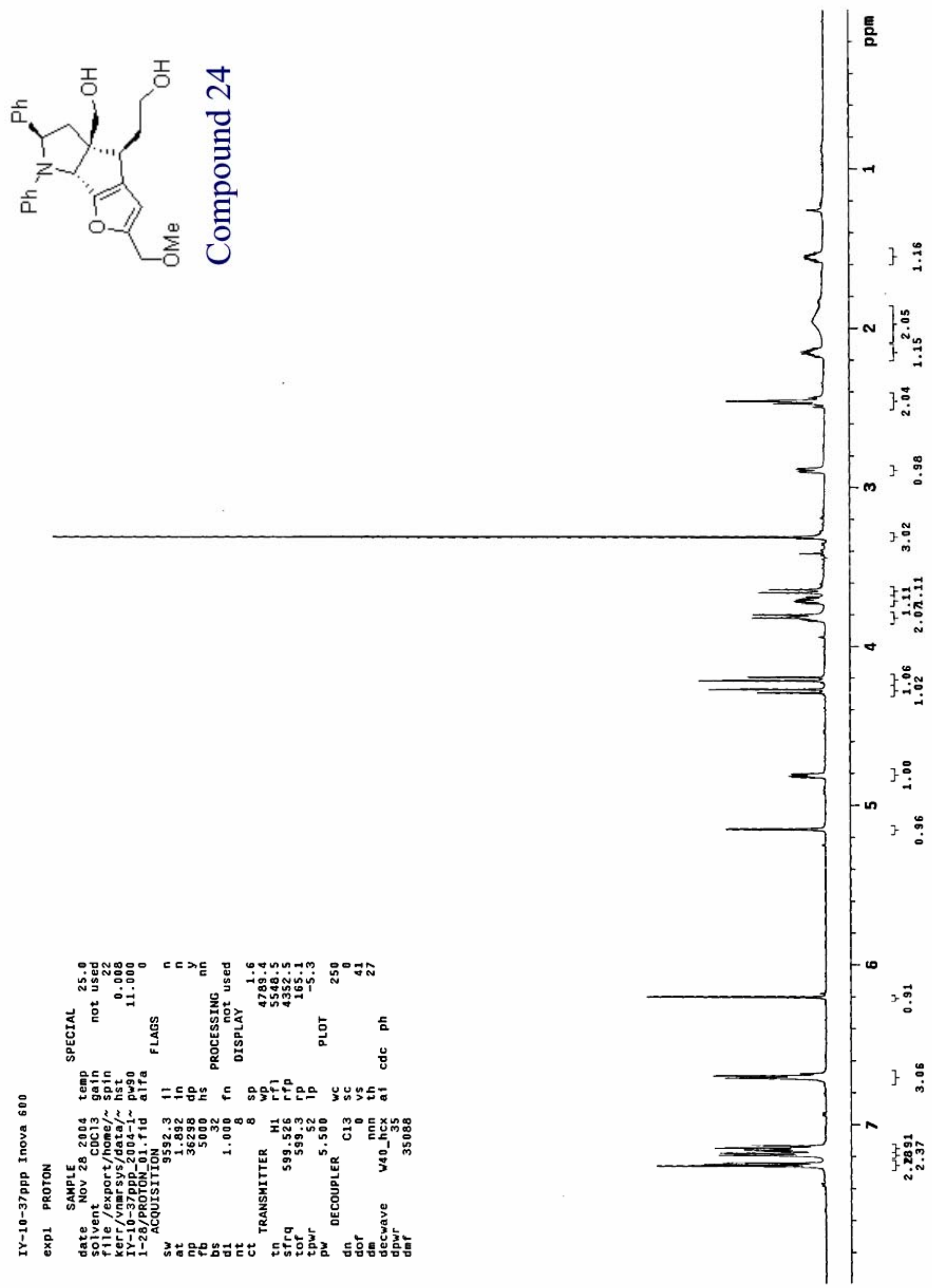

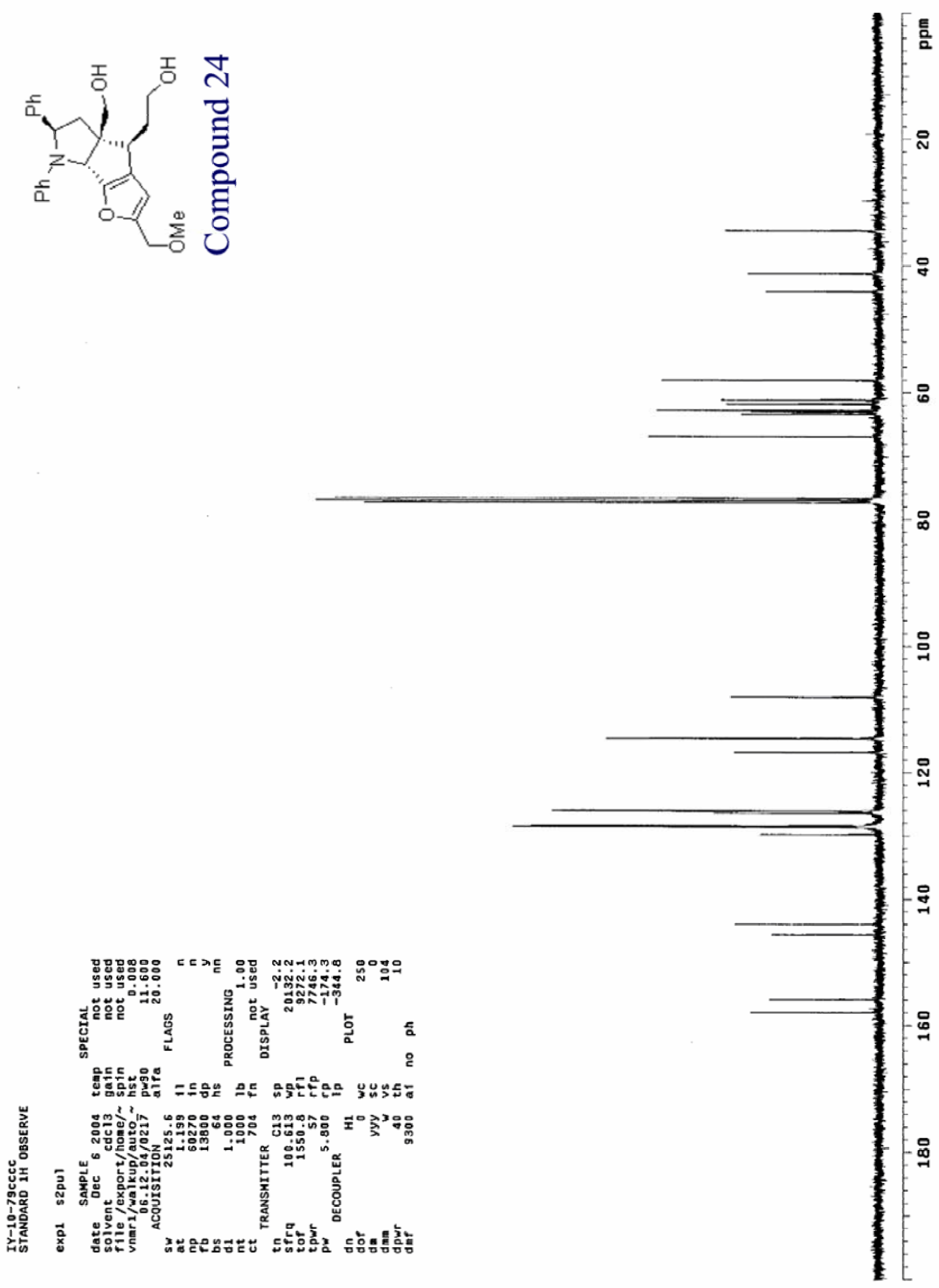


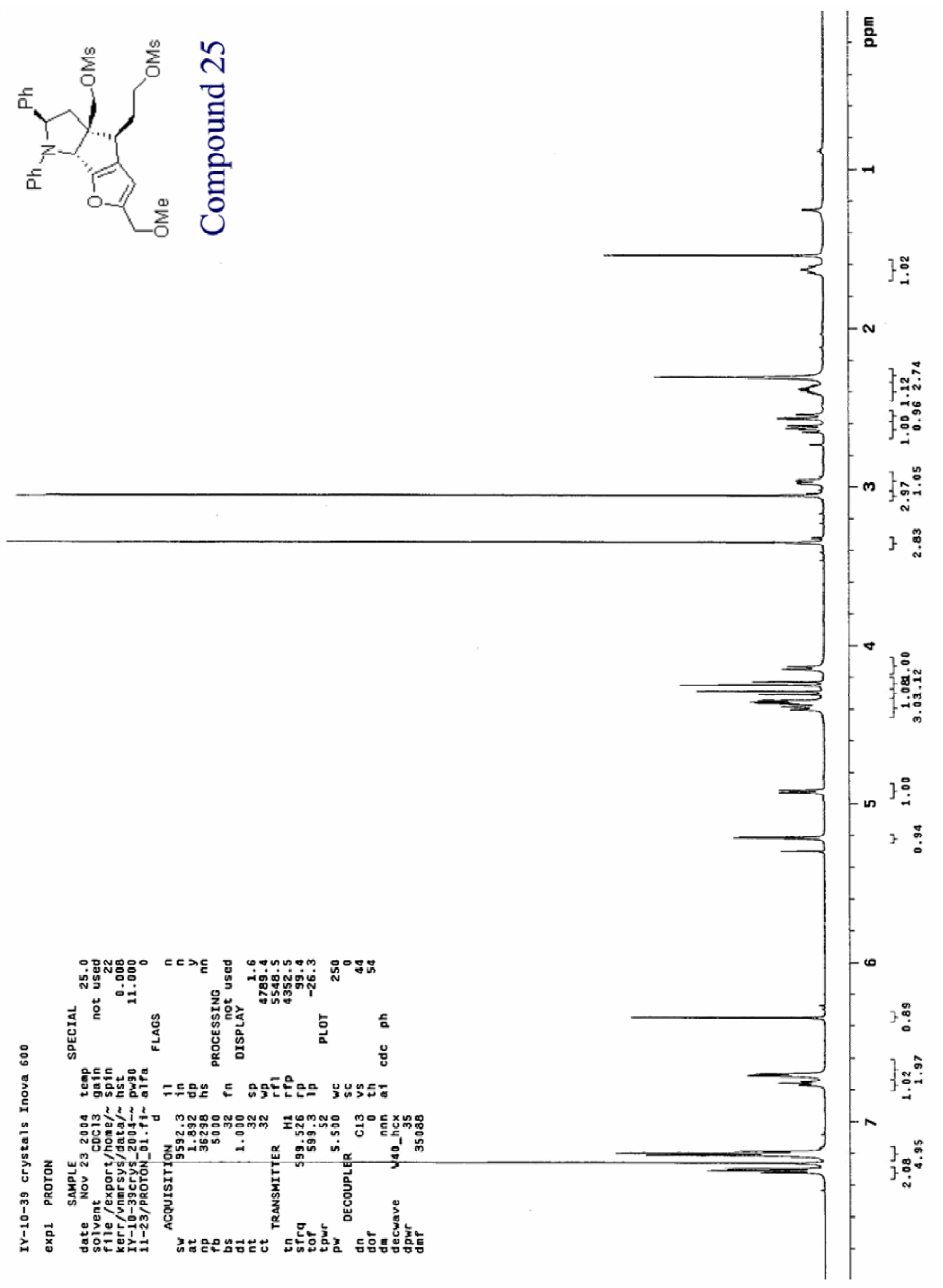


<smiles>[O]Cc1cc2c(o1)[C@H]1[C]([O])[C@@H]([O])C[C@@]1(CO)[C@@H]2CCO</smiles>

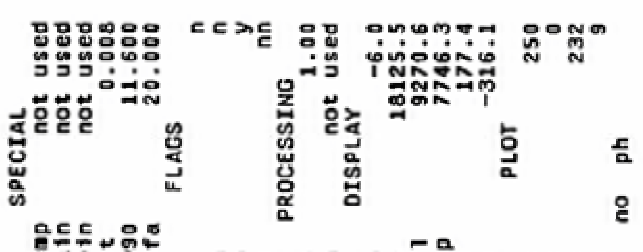

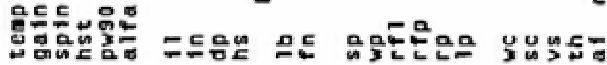

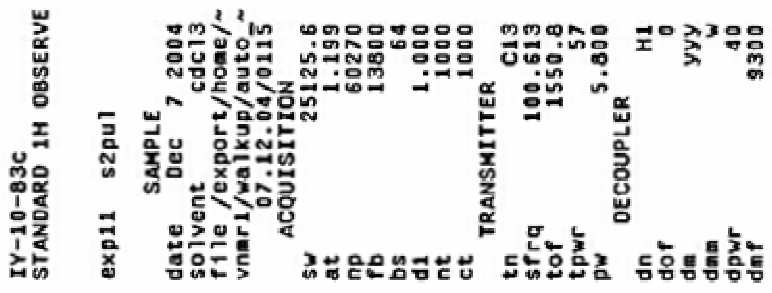



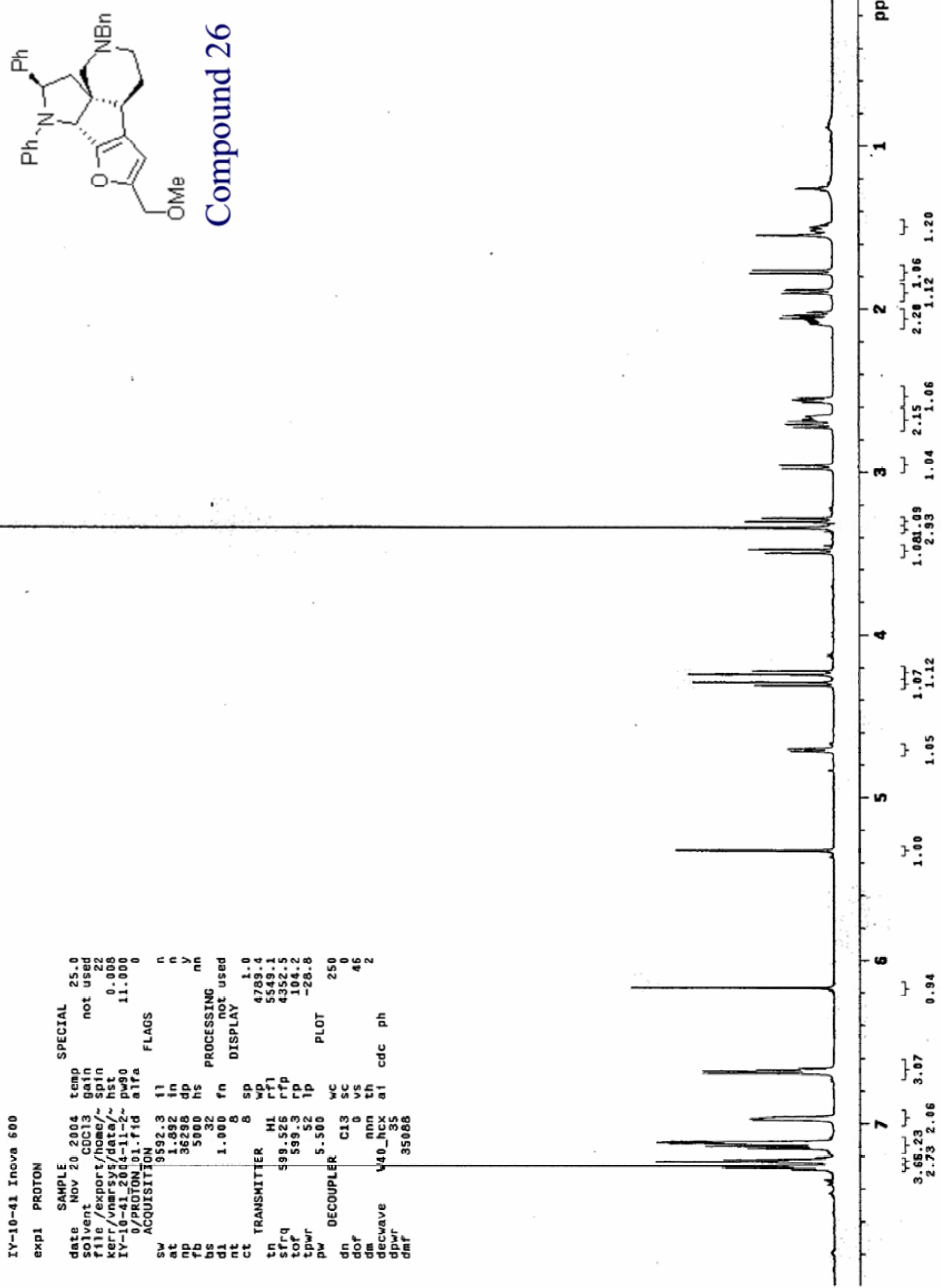

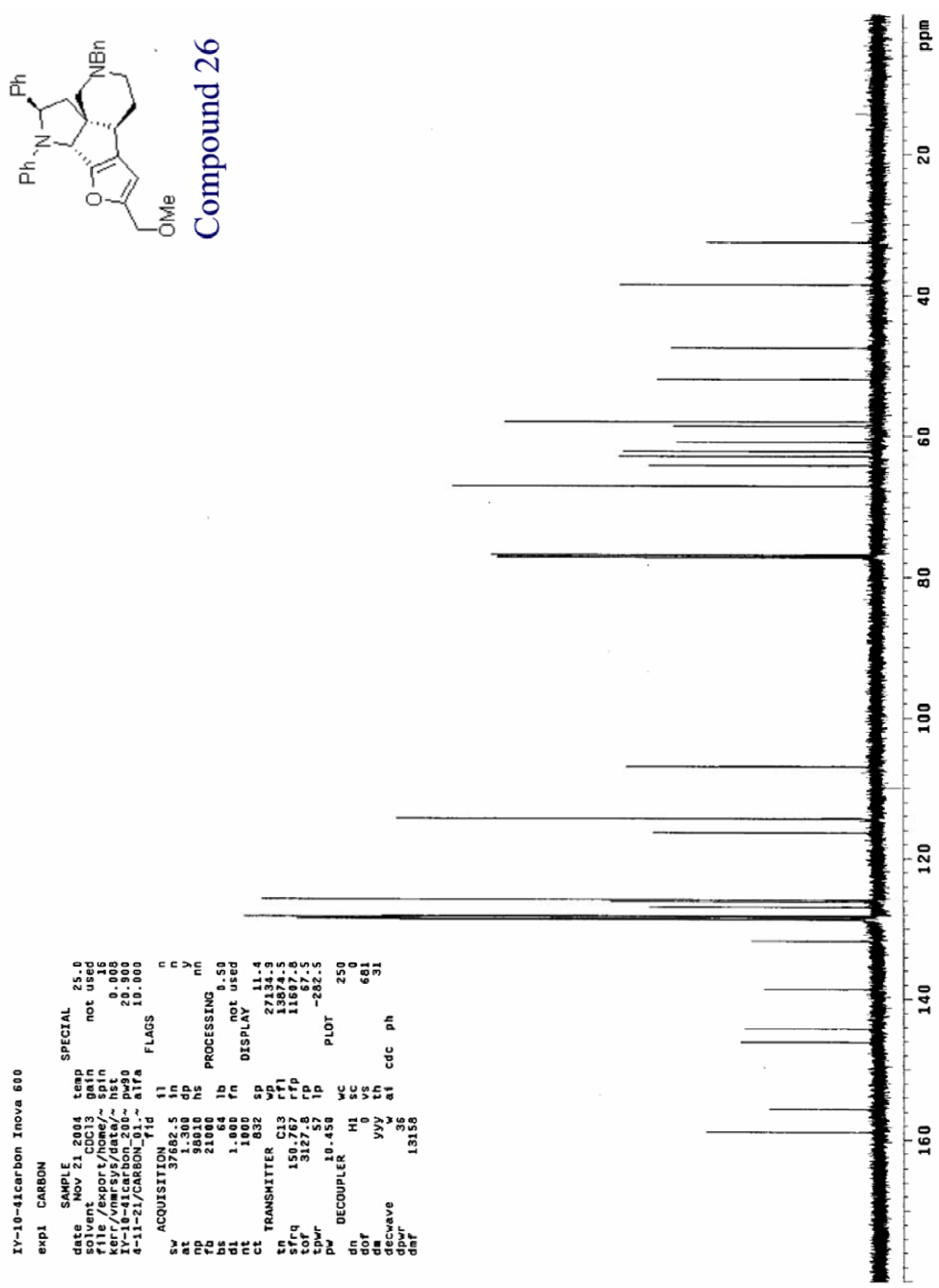